\title{
Inside the bottleneck. Stimulus competition and selection in visual and phonological encoding.
}

Citation for published version (APA):

Bles, M. (2007). Inside the bottleneck. Stimulus competition and selection in visual and phonological encoding. [Doctoral Thesis, Maastricht University]. Datawyse / Universitaire Pers Maastricht. https://doi.org/10.26481/dis.20070701mb

Document status and date:

Published: 01/01/2007

DOI:

$10.26481 / \mathrm{dis} .20070701 \mathrm{mb}$

Document Version:

Publisher's PDF, also known as Version of record

\section{Please check the document version of this publication:}

- A submitted manuscript is the version of the article upon submission and before peer-review. There can be important differences between the submitted version and the official published version of record.

People interested in the research are advised to contact the author for the final version of the publication, or visit the DOI to the publisher's website.

- The final author version and the galley proof are versions of the publication after peer review.

- The final published version features the final layout of the paper including the volume, issue and page numbers.

Link to publication

\footnotetext{
General rights rights.

- You may freely distribute the URL identifying the publication in the public portal. please follow below link for the End User Agreement:

www.umlib.nl/taverne-license

Take down policy

If you believe that this document breaches copyright please contact us at:

repository@maastrichtuniversity.nl

providing details and we will investigate your claim.
}

Copyright and moral rights for the publications made accessible in the public portal are retained by the authors and/or other copyright owners and it is a condition of accessing publications that users recognise and abide by the legal requirements associated with these

- Users may download and print one copy of any publication from the public portal for the purpose of private study or research.

- You may not further distribute the material or use it for any profit-making activity or commercial gain

If the publication is distributed under the terms of Article $25 \mathrm{fa}$ of the Dutch Copyright Act, indicated by the "Taverne" license above, 


\section{Inside the bottleneck}

Stimulus competition and selection in visual and phonological encoding

Mart Bles 
Colophon:

Cover design: Hein Bles

Layout: Mart Bles \& Hein Bles

Production: Datawyse / Universitaire Pers Maastricht

Copyright (C) Mart Bles, Berlin, 2007

ISBN: 978-90-5278-666-7 


\section{Inside the bottleneck}

Stimulus competition and selection in visual and phonological encoding

\section{PROEFSCHRIFT}

ter verkrijging van de graad van doctor aan de Universiteit Maastricht, op gezag van de Rector Magnificus, Prof. mr. G.P.M.F. Mols

volgens het besluit van het College van Decanen, in het openbaar te verdedigen

op donderdag 1 November 2007 om 14.00 uur

door

Martinus Bles

Geboren op 2 Juni 1980 te Eersel

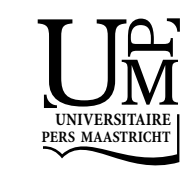




\section{Promotor:}

Prof. dr. R. Goebel

\section{Copromotor:}

Dr. B.M. Jansma

\section{Beoordelingscommissie:}

Prof. dr. G. Kok (voorzitter)

Dr. M. Bonte

Prof. dr. J. Jolles

Dr. L. Jonkman

Prof. dr. P. Zwitserlood (Westfälische Wilhelms-Universität Münster) 


\section{Contents}

1. General introduction $\quad 7$

Visual selection mechanisms in humans 9

The time course of competitive and biasing mechanisms 10

Selection beyond the visual domain $\quad 10$

Linguistic selection in the absence of visual selection 12

$\begin{array}{ll}\text { References } & 13\end{array}$

2. Receptive field size-dependent attention effects in simultaneously $\begin{array}{ll}\text { presented stimulus displays } & 17\end{array}$

$\begin{array}{ll}\text { Introduction } & 18\end{array}$

Methods $\quad 22$

Results $\quad 25$

References $\quad 28$

3. The time course of competitive and biasing mechanisms in human visual cortex $\quad 31$

Introduction $\quad 32$

Methods $\quad 36$

Results $\quad 38$

Discussion $\quad 43$

References 46

4. Phonological processing of ignored distractor pictures, an fMRI investigation $\quad 51$

Introduction 52

Methods $\quad 57$

Results 61

Discussion $\quad 65$

Relatedness effects 66

Hemispheric lateralisation and task-specific activations 67

$\begin{array}{ll}\text { References } & 70\end{array}$

Appendix 4.1 74

5. Neural aspects of cohort-size reduction during visual gating $\quad 77$

$\begin{array}{ll}\text { Introduction } & 78\end{array}$

Methods $\quad 82$

Results $\quad 85$

Discussion $\quad 90$

$\begin{array}{ll}\text { P2 time window } & 91\end{array}$ 
N400 time window 93

References 96

6. General discussion 103

Biased competition in human visual cortex 104

Fate of ignored stimuli 106

Selection in the absence of visual selection $\quad 109$

References

Summary

115

Samenvatting

119

Acknowledgments

122

Curriculum vitae

125 
General introduction 
A typical property of human perception and behaviour, is its selective nature. Of all the stimuli presented to us in an everyday situation, only few ever reach our awareness. We all know the situation of walking on a busy street and failing to notice a well-known person walking by. A more extreme example is given by an experiment in which participants watched a video of people who were passing along a ball amongst themselves. Their task was to count how often the members of a team passed the ball to each other. This task turned out to be so demanding, that approximately half of the participants failed to notice a person in a gorilla suit walking through the scene (Simons \& Chabris, 1999). The fact that we do not become aware of all information presented to us indicates that, somewhere between our sensory and executive systems, a selection is being made that prioritizes the processing of some types of information, while diminishing that of others. It is generally believed that the necessity for these selections is the limited capacity of our cognitive systems. That is, there is a bottleneck in the system through which only a limited amount of information can flow. This flow is regulated by a selective process through which the brain chooses some stimuli for further processing, while ignoring others, a process which is generally referred to as attention.

In part, the limitation of our perceptual systems is thought to originate from the level of the receptive fields (RF) in single neurons. The RF is defined as the area of the perceptual (e.g. visual) space in which a stimulus needs to be presented in order to influence a neuron's firing rate. When a stimulus falls within the RF, the neuron's firing rate will reflect the properties of that stimulus. For example, a given neuron may fire rapidly in response to a line of a certain orientation (this would be a 'good' stimulus for this neuron), but less rapidly to lines of different orientations ('poor' stimulus) (Hubel \& Wiesel, 1968). From the firing rate of the neuron, it can then be deducted what the orientation of the presented stimulus was. This mechanism can, up to some point, nicely explain human object perception in case of single stimuli. However, a problem arises when, for example, a good and poor stimulus are presented to the RF of a neuron. In this case the stimuli start competing for neural representation, since the neuron cannot fully represent both stimuli simultaneously (i.e. it cannot fire rapidly and slowly at the same time). When a good and a poor stimulus are presented together within the RF of a macaque V2 or V4

8 neuron, it responds with a firing rate that is a weighted average of the firing rates evoked by the single stimuli (e.g. Chelazzi et al., 1998; Chelazzi et al., 2001; Luck et al., 1997; Miller et al., 1993; Moran \& Desimone, 1985; Recanzone et al., 1997; Reynolds et al., 1999). Thus, instead of being processed independently, multiple stimuli within the RF of a neuron can be seen as mutually suppressing each other's representation and competing for neural resources.

The competition between stimuli can be biased by attending one of them. When one of the stimuli is attended, the neuron's firing rate reflects the presence of the attended stimulus. If the effective stimulus is attended, the neuron's firing rate increases to a level 
that is approximately the same as when the effective stimulus is presented alone. Likewise, if the ineffective stimulus is attended, the firing rate decreases to the level that can be observed when the ineffective stimulus is presented alone (Chelazzi et al., 1998; Chelazzi et al., 2001 ; Luck et al., 1997; Moran \& Desimone, 1985; Reynolds et al., 1999). These results imply that attention does not merely enhance responses to attended stimuli. Rather, in the case of multiple stimuli, attention filters out the influence of surrounding stimuli. The processing of the attended stimulus gains precedence over that of others and the neuron's firing rate will reflect the presence of the attended stimulus and not of the distractors.

\section{Visual selection mechanisms in humans}

Due to its invasive nature, single-cell recordings are not a suitable technique to investigate cortical functioning in humans. Instead, neuroimaging techniques like functional Magnetic Resonance Imaging (fMRI) have become increasingly popular over the last decades. With fMRI, increases in oxygen-rich blood flow can be measured at thousands of locations in the brain simultaneously. From the amount of blood-flow increase, it is deducted how active a given area was during the measurement.

Recently, some fMRI studies have found indications that biased competition may also be a dominant selection mechanism in human visual cortex (Kastner et al., 1998; 1999; 2001 a; Pinsk et al., 1999). In these experiments, participants viewed displays of four complex, colourful stimuli that were presented either simultaneously or sequentially. It was reasoned that in the simultaneous condition, stimuli would compete for neural representation since visual areas have to process the information of several stimuli at the same time. In the sequential condition, instead, there is only one stimulus presented at a time, preventing competition between stimuli. Indeed, the activity in participants' visual cortex was decreased when stimuli were presented simultaneously as compared to the signal obtained when stimuli were presented sequentially. This was interpreted as evidence that during simultaneous stimulation the neural representations of the different stimuli interact in a suppressive way and that no such competition takes place in sequential stimulation (Kastner et al., 1998; 2001a). Moreover, suppression effects were not observed when participants attended one of the stimuli, suggesting that attention biased the competition in favour of the attended item, relieving it from the suppressive effects of the surrounding stimuli (Kastner et al., 1998; 1999).

A potential drawback of the method described above is that by comparing the activation in the sequential condition with that in the simultaneous condition, a higher amount of transient stimulus-onsets is present in sequential trials as opposed to simultaneous trials. It has been shown that a higher amount of stimulus-onsets can lead to higher activation levels in visual cortex (Fox \& Raichle, 1985; Rees et al., 1997), which could form a potential confound in the interpretation of the results in light of a biased competition 
framework. These issues are addressed in chapter 2, and a new design is introduced that removes the need for the 'sequential' condition, while retaining the possibility to test hypotheses specific to a biased competition account.

\section{The time course of competitive and biasing mechanisms}

The observations reported in chapter 2 support the biased competition account, in that suppressive effects were observed in areas where RF sizes are large enough to encompass multiple stimuli, as well as the observation that these effects disappear when one of the stimuli is attended. However, the question then remains what the time course is of both the suppressive effects and the biasing signal. Some fMRI studies have shown attentional increases in V1 (Di Russo et al., 2003; Martinez et al., 1999; 2001), the earliest cortical area for visual processing. This suggests that any competition between stimuli is biased from stimulus onset and in the earliest visual areas. However, although fMRI is an excellent method for localizing cognitive processes in the brain at a sub-centimetre scale, it has a time resolution in the order of seconds. This makes it less suited for studying the timing of these process, which typically occur within a tens of milliseconds from each other.

Event-Related Potential (ERP) studies measure electrical potentials generated by the brain in response to cognitive events. Whereas with this method, it is difficult to localize any effects at a scale below several centimetres, it has an excellent temporal resolution, typically measuring cortical potentials every few milliseconds. ERP studies have shown that the attention effects in $\mathrm{V} 1$ were not present from stimulus onset, but rather started to appear in a later re-entrant sweep of activity through V1 (Di Russo et al., 2003; Martinez et al., $1999 ; 2001)$. This implies that it takes time for attention to bias the competition between stimuli, meaning that there should be an observable time in which stimuli compete with each other. In chapter 3, the design that was introduced in chapter 2 was employed in an ERP study which served to investigate the timing of suppression effects and the biasing signal in visual cortex.

\section{Selection beyond the visual domain}

Traditionally, attention research has focused on the visual domain. However, selections take place across sensory domains, and also in higher-order cognitive domains like memory, language, or motor execution. That is, only a limited amount of contents from our memory can be active in working memory at one time (Miller, 1956) and the execution of motor programs is severely hampered if more than one program is executed simultaneously (Baldissera et al., 1991). The same holds for the language system. People are often encountered with multiple language stimuli: in a room full of people, more than one person may talk simultaneously, electronic media and bill boards may present 
us with simultaneous language input, and the mere act of reading bombards our brain with hundreds of words per minute. In a similar way, when we have the intention to utter a thought, a choice needs to be made about the contents of the utterance, the exact phrasing, and which words are used to refer to objects or persons. As is true for visual stimuli, not every language stimulus we encounter reaches our awareness. When people are asked to attend an auditory stream in one ear while ignoring the stream in the other, they are generally unable to report any characteristics of the unattended stream (Broadbent, 1958; Cherry, 1953). Similarly, we are often unaware of the alternative ways to express a thought (for example using the word 'couch', or simply 'it', instead of 'sofa' to delineate the thing we are sitting on). Thus, selective processes seem to be at work across cognitive domains. In chapter 4, we will focus on selection during language production, by making use of a silent picture naming. The use of this paradigm served investigate a) selection processes in the language domain, and b) the fate of ignored visual stimuli.

The fact that our brain selects certain stimuli for further processing while ignoring others, raises the question what exactly happens to the ignored stimuli. This is crucial for understanding attentional mechanisms in humans. Traditionally, there has been a strong debate between proponents of early selection theorists, who state that selection takes place at an early, perceptual level, and late selection theorists, who argue that every stimulus in a display is perceptually processed and identified, but selection takes place at a higher, executive level. Attempts to resolve this discussion have led to propositions like the perceptual load theory, which posits that the level of selection depends on the complexity of the task and the stimulus display (Lavie, 1995). That is, in cases where our limitedresource perceptual system is overloaded, selection takes place at an early level. But when only a few, simple stimuli have to be processed, unattended stimuli are automatically processed and identified, and selection takes place at a later stage.

However, there is a long processing stream between early sensory areas and the final awareness of a stimulus or the overt behavioural response to it. In picture naming, for example, after the visual features of the picture are encoded, the proper conceptual representation of the depicted object needs to be activated, along with its syntactic properties, its phonology, and the correct articulatory program. This stream involves an extensive network of left-hemispheric cortical areas (Indefrey \& Levelt, 2004). In principle, selection could take place within any of these areas. It is unknown whether (weakened) neuronal representation of ignored stimuli are activated within these areas and, especially, whether the extracted features of a distractor stimulus depend on the task that is performed. The design that was employed in chapter 4 was a modified version of that used in chapters 2 and 3 , and served to investigate what the fate of the ignored distractor pictures was. Instead of using abstract pictures, stimuli now consisted of drawings of common objects. Since these objects have linguistic properties (e.g. they have a meaning and a sound), we could explore whether these properties were analyzed by cortical areas involved in their 
processing, even if the picture itself was to be ignored. In addition, this design enabled us to investigate the nature of selective processes within linguistic cortical areas: how do these areas cope with the presentation of multiple stimuli? Are there similarities with the well-studied selection mechanisms in the visual system?

\section{Linguistic selection in the absence of visual selection}

The design used in chapter 4 revealed that the nature of the selection process in linguistic areas depended on the task participants were performing. The results showed that, at least under some circumstances, linguistic properties of ignored distractors are fully identified. Importantly, these results have to be viewed in light of the selective processes at a visual level that are described in chapters 2 and 3 . Since stimuli already compete at a visual level, the representations of distractor stimuli that are forwarded to higher-order processing areas are likely to be attenuated in comparison with that of attended stimuli. Therefore, if competition between these stimuli takes place in linguistic processing areas, it is biased towards the linguistic representation of the attended stimulus, which reduces the likelihood to detect competitive effects in these areas.

The goal of chapter 5 was to introduce a design that allowed linguistic selection to be investigated without this dependency on interactions in the visual domain. Participants viewed a letter projected on the screen (e.g. ' $C$ '). After a while, another letter appeared to the right of this letter ('CA'), followed by another one ('CAL'). The letters on the screen spelled out parts of Dutch words, which we expected to be automatically activated in participants' mental lexicons (Johnson \& Pugh, 1994; Marslen-Wilson, 1987). After the presentation of a new letter, some of the activated words were not compatible with the input anymore, and needed to be inhibited. E.g. if the letters ' $c$ ' and ' $a$ ' are present on the screen, this is consistent with words like 'calf', 'car', 'cat', 'cab', 'call' etc. Adding the letter ' $I$ ' to the display leaves only 'calf' and 'call' as possibilities, and the other words need to be inhibited. Stimuli were created such that the amount of words that needed to be inhibited was either large, medium or small. In chapter 5, ERP data are described that reflect the inhibition of potential lexical candidates. Finally, a general discussion of

12 the previously described chapters is presented in chapter 6, along with implications and future directions. 


\section{References}

Baldissera, F., Cavallari, P., Marini, G., \& Tassone, G. (1991). Differential control of inphase and anti-phase coupling of rhythmic movements of ipsilateral hand and foot. Exp Brain Res 83, 375-380.

Broadbent, D. (1958). Perception and communication (London, Pergamon Press).

Chelazzi, L., Duncan, J., Miller, E. K., \& Desimone, R. (1998). Responses of neurons in inferior temporal cortex during memory-guided visual search. Journal of Neurophysiology 80, 2918-2940.

Chelazzi, L., Miller, E. K., Duncan, J., \& Desimone, R. (2001). Responses of Neurons in Macaque Area V4 During Memory-guided visual Search. Cerebral Cortex 11, 761-772.

Cherry, E. C. (1953). Some experiments on the recognition of speech, with one and with two ears. The Journal of the Acoustical Society of America 25, 975-979.

Di Russo, F., Martinez, A., \& Hillyard, S. A. (2003). Source analysis of event-related cortical activity during visuo-spatial attention. Cerebral Cortex 13, 486-499.

Fox, P. T., \& Raichle, M. E. (1985). Stimulus rate determines regional brain blood flow in striate cortex. Ann Neurol 17, 303-305.

Hubel, D. H., \& Wiesel, T. N. (1968). Receptive fields and functional architecture of monkey striate cortex. Journal of Physiology 195, 215-243.

Indefrey, P., \& Levelt, W. J. (2004). The spatial and temporal signatures of word production components. Cognition 92, 101-144.

Johnson, N. F., \& Pugh, K. R. (1994). A cohort model of visual word recognition. Cognit Psychol 26, 240-346.

Kastner, S., De Weerd, P., Desimone, R., \& Ungerleider, L. (1998). Mechanisms of Directed Attention in the Human Extrastriate Cortex as Revealed by Functional MRI. Science 282, 108-111.

Kastner, S., Pinsk, M. A., De Weerd, P., Desimone, R., \& Ungerleider, L. (1999). Increased Activity in Human Visual Cortex during Directed Attention in the Absence of Visual Stimulation. Neuron 22, 751-761.

Kastner, S., De Weerd, P., Pinsk, M. A., Elizondo, M. I., Desimone, R., \& Ungerleider, L. (2001a). Modulation of Sensory Suppression: Implications for Receptive Field Sizes in the Human Visual Cortex. Journal of Neurophysiology 86, 1398-1411.

Lavie, N. (1995). Perceptual load as a necessary condition for selective attention. J Exp Psychol Hum Percept Perform 21, 451-468. 
Luck, S. J., Chelazzi, L., Hillyard, S. A., \& Desimone, R. (1997). Neural mechanisms of spatial selective attention in areas $\mathrm{V} 1, \mathrm{~V} 2$, and $\mathrm{V} 4$ of macaque visual cortex. J Neurophysiol 77, 24-42.

Marslen-Wilson, W. D. (1987). Functional parallelism in spoken word-recognition. Cognition 25, 71-102.

Martinez, A., Anllo-Vento, L., Sereno, M. I., Frank.L.R., Buxton, R. B., Dubowitz, D. J., Wong, E. C., Heinze, H. J., \& Hillyard, S. A. (1999). Involvement of striate and extrastriate visual cortical areas in spatial attention. Nature Neuroscience 2, 364369.

Martinez, A., DiRusso, F., Anllo-Vento, L., Sereno, M. I., Buxton, R. B., \& Hillyard, S. A. (2001). Putting spatial attention on the map: timing and localization of stimulus selection processes in striate and extrastriate visual areas. Vision Res 41, 1437 1457.

Miller, E. K., Gochin, P. M., \& Gross, C. G. (1993). Suppression of visual responses of neurons in inferior temporal cortex of the awake macaque by addition of a second stimulus. Brain Res 616, 25-29.

Miller, G. A. (1956). The magical number seven, plus or minus two: some limits on our capacity for processing information. Psychological Review 63, 81-97.

Moran, J., \& Desimone, R. (1985). Selective attention gates visual processing in the extrastriate cortex. Science 229, 782-784.

Pinsk, M. A., Kastner, S., Desimone, R., \& Ungerleider, L. (1999). An estimate of receptive field sizes in human visual cortex. Neurolmage 9, s885.

Recanzone, G. H., Wurtz, R. H., \& Schwarz, U. (1997). Responses of MT and MST neurons to one and two moving objects in the receptive field. J Neurophysiol 78, 29042915.

Rees, G., Howseman, A., Josephs, O., Frith, C. D., Friston, K. J., Frackowiak, R. S., \& Turner, R. (1997). Characterizing the relationship between BOLD contrast and regional cerebral blood flow measurements by varying the stimulus presentation rate. Neuroimage 6, 270-278.

Reynolds, J. H., Chelazzi, L., \& Desimone, R. (1999). Competitive mechanisms subserve attention in macaque areas V2 and V4. J Neurosci 19, 1736-1753.

Simons, D. J., \& Chabris, C. F. (1999). Gorillas in our midst: sustained inattentional blindness for dynamic events. Perception 28, 1059-1074. 

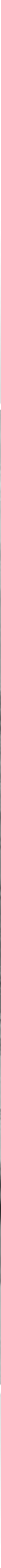


\section{Receptive field size-dependent attention effects in simultaneously presented stimulus displays}

Neurophysiological studies in monkeys show that multiple stimuli presented within the receptive field of a neuron are not processed independently, but rather act in a mutually suppressive way. Recently, such suppressive interactions have also been reported in human neuroimaging studies. This is seen as evidence that stimuli compete for neural representation. According to the 'biased competition' approach, attention can bias this competition in favor of the attended stimulus, relieving it of the suppressive influences of the distracters. In this paper, we report data that support these findings. Specifically, the effect of attention on stimuli with different spatial separations was investigated more thoroughly. The biased competition approach would predict that, for a given spatial separation and eccentricity, the difference between attended and unattended displays depends on the receptive field size of an area. In a blocked fMRI experiment, participants viewed 4 simultaneously presented, colorful pictures under different attention conditions (attended and unattended). Stimuli were separated either $2^{\circ}, 4^{\circ}$ or $7^{\circ}$. In line with previous experiments we found that the effect of attention correlated with the estimated receptive field size of an area. In areas V1, V2 and VP, where estimated receptive field sizes are small, no significant attention effects were found in any of the spatial separation conditions. In V4, there was a significant difference between attended and unattended conditions for the $2^{\circ}$ and $4^{\circ}$ spatial separations, but not for $7^{\circ}$. Finally in area TEO, significant differences between attended and unattended conditions were observed for all spatial separations. 


\section{Introduction}

Only a few of the objects presented to us in a typical visual scene are behaviorally relevant. Our brain selects these stimuli for further processing and filters out other, unwanted information through a process referred to as attention (Broadbent, 1958; Treisman, 1969). The present research project aimed at further investigating the mechanisms that underlie stimulus selection. More specifically, we tested hypotheses derived from a 'biased competition' account of visual attention (for an overview, see Desimone and Duncan, 1995; Kastner and Ungerleider, 2001 b), which has been based primarily on neurophysiological studies in animals (e.g. Chelazzi et al., 1998; Luck et al., 1997; Moran and Desimone, 1985; Reynolds et al., 1999), but which has recently received support from human neuroimaging studies (Kastner et al., 1998; Kastner et al., 1999; Kastner et al., 2001 a; Pinsk et al., 1999).

Evidence from single-cell recordings in extrastriate cortex indicates that whenever multiple stimuli are presented within the receptive field (RF) of a neuron in the absence of attention, these stimuli compete for control over that neuron's firing rate. When, for example, an effective and an ineffective stimulus are presented together within the RF of a macaque V2 or V4 neuron, the cell responds with a firing rate that is a weighted average of the firing rates evoked by the single stimuli (Chelazzi et al., 1998; Chelazzi et al., 2001 ; Luck et al., 1997; Miller et al., 1993; Moran and Desimone, 1985; Recanzone et al., 1997; Reynolds et al., 1999). Thus, instead of being processed independently, multiple stimuli within the RF of a neuron can be seen as mutually suppressing each other's representation and competing for neural resources.

The competition between stimuli can be biased by attending one of them. When one of the stimuli is attended, the neuron's firing rate reflects the presence of the attended stimulus. If the effective stimulus is attended, the neuron's firing rate increases to a level that is approximately the same as when the effective stimulus is presented alone. Likewise, if the ineffective stimulus is attended, the firing rate decreases to the level that can be observed when the ineffective stimulus is presented alone (Chelazzi et al., 1998; Chelazzi et al., 2001; Luck et al., 1997; Moran and Desimone, 1985; Reynolds et al., 1999). Clearly, attention does not merely enhance responses to attended stimuli. Rather, in the case of multiple stimuli, attention filters out the influence of surrounding stimuli. The processing of the attended stimulus gains precedence over that of others and the neuron's firing rate will reflect the presence of the attended stimulus and not of the distracters.

Similarly, Kastner et al. (1998; 1999; 2001a) found evidence for competitive interactions between stimuli in the human visual cortex. In an fMRI experiment, participants saw displays of 4 complex, colorful, visual stimuli. These stimuli were either presented simultaneously or sequentially. In the simultaneous condition stimuli compete for neural representation since visual areas have to process the information of several stimuli at 
the same time. In the sequential condition there is only one stimulus presented at a time, preventing competition between stimuli. The BOLD response in participants' visual areas V2/VP, V4 and TEO was decreased when stimuli were presented simultaneously as compared to the signal obtained when stimuli were presented sequentially. The authors interpreted these findings as evidence that during simultaneous stimulation the neural representations of the different stimuli interact in a suppressive way and that no such competition takes place in sequential stimulation (Kastner et al., 1998; Kastner et al., 2001 a). Moreover, suppression effects were not observed when participants attended one of the stimuli, suggesting that attention biased the competition in favor of the attended item, relieving it from the suppressive effects of the surrounding stimuli (Kastner et al., 1998; Kastner et al., 1999).

As visual areas are located further up the cortical visual processing stream, their average RF size increases. As a consequence, on average more stimuli will be present within the RFs of higher order areas compared to lower order areas, given an identical display. This leads to the prediction that stronger competitive interactions between stimuli should be observed in higher order areas, since neurons in these areas have larger RFs (see figure 2.1). Indeed, Kastner et al. $(1998 ; 1999 ; 2001$ a) observed that the competitive interactions were not of the same magnitude in all visual areas. No competition effects were found in $\mathrm{V} 1$, where RF sizes are so small that no stimuli will simultaneously fall within the same RF. However, competitive interactions were observed in V2/NP and this effect was even more pronounced in more anterior areas like V4 and TEO. In line with the biased competition theory, this suggests that the magnitude of the competitive interaction effect is scaled with RF size of an area.

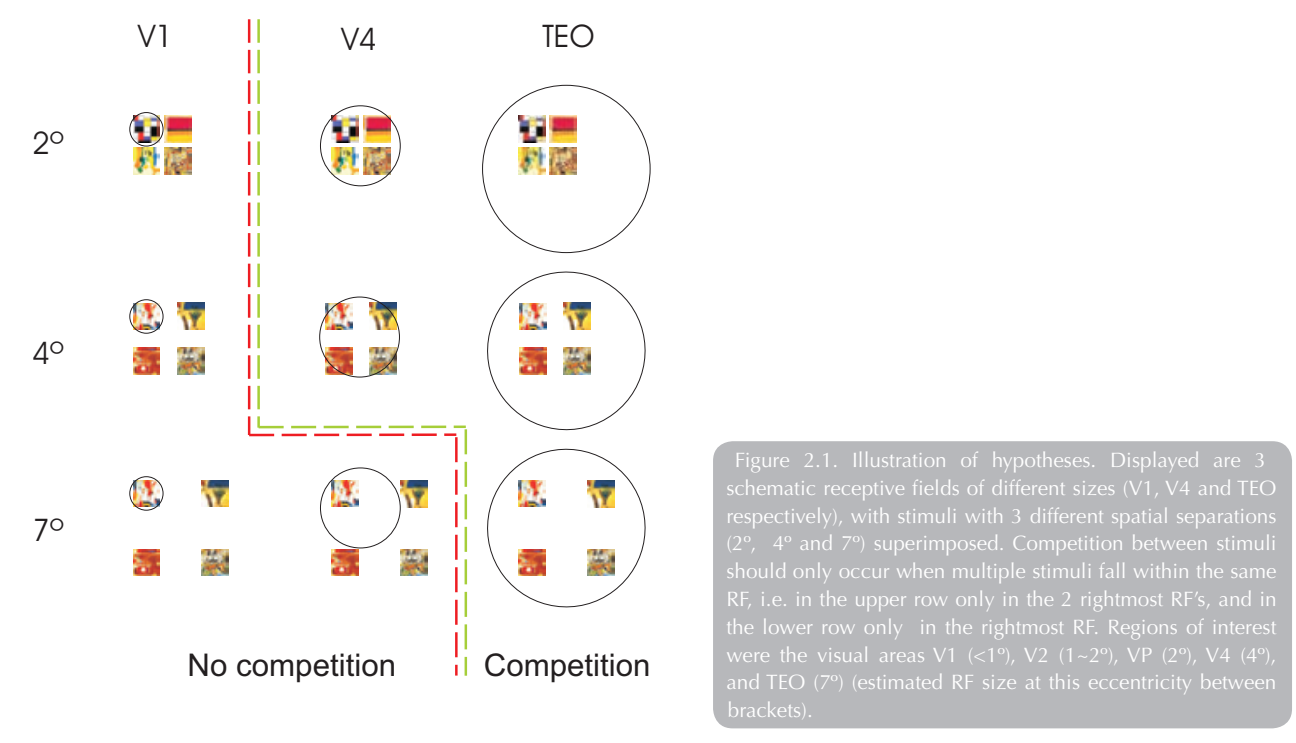


Further evidence that competitive interactions between stimuli are dependent on RF size was found in a follow-up study (Kastner et al., 2001a; Pinsk et al., 1999). In this study the spatial separation between the stimuli in the display was systematically varied. By placing stimuli further apart, less of them will fall within the same RF (see figure 2.1) and therefore weaker competitive interactions should be observed. Indeed, the magnitude of the suppressive interactions could be decreased within areas by increasing stimulus separation. The degree to which display spacing affected suppressive interactions decreased as areas were placed higher up the visual stream, where RF sizes increase.

A potential drawback of the method described above is that by comparing the activation in the sequential condition with that in the simultaneous condition, a higher amount of transient stimulus-onsets is present in sequential trials as opposed to simultaneous trials. Because the magnitude of the hemodynamic response is dependent on the number of transient stimulus onsets (Fox and Raichle, 1985; Price et al., 1996; Rees et al., 1997; Schneider et al., 1994), the higher BOLD-signal change in sequential conditions might be explained by the higher number of stimulus onsets, rather than a reduced amount of suppression (Kastner et al., 1998; Kastner et al., 2001a). There are, however, several arguments why this seems unlikely. First of all, in a control condition in which the stimulation rate was kept constant, distracter stimuli placed in the lower visual field were shown to suppress the response to a stimulus presented alone in the upper visual field, indicating that it was not presentation rate, but distracter presence which controlled the amount of suppression (see Kastner et al. (2001a) for more details). Furthermore, if the higher number of transient onsets would be the cause of the increased BOLD signal change in the sequential condition, then this effect should be relatively equal over visual areas and spatial separations (Rees et al., 1997; Schneider et al., 1994). Instead, Kastner et al. $(1998 ; 2001$ a) found that the size of the suppression effect was dependent on RF size of an area and the amount of separation between stimuli. Similarly, if the amount of transient onsets explained the difference between the simultaneous and the sequential condition, a similar attention effect should have been present in the simultaneous and the sequential conditions (Rees et al., 1997). Instead, when comparing simultaneously presented with sequentially presented items, attention effects in V4 were larger in the 20 simultaneous condition (Kastner et al., 1998).

So far, the studies by Kastner et al. are the only ones which demonstrate biased competition in humans. In these studies, attention effects for simultaneously presented stimuli were only investigated in V1 and V4 and only for one spatial separation (Kastner et al., 1998). The role of spatial separation was only investigated by looking at the difference between simultaneous and sequential conditions in the absence of attention (Kastner et al., 2001a). Our goal was to replicate and extend these findings. We chose a design which on the one hand investigates the role of attention in displays of various spatial separations more thoroughly, while on the other hand removing the need for the 
'sequential' condition. In the present study the factors attention, spatial separation and visual area were completely crossed, which enabled us to gain a more comprehensive look on the interactions between RF size, competition and the effects of selective attention. In a modified version of the block design used by Kastner et al. (1998; 1999; 2001a; Pinsk et al., 1999), participants viewed 4 abstract, colorful stimuli, that were always simultaneously presented in the upper right quadrant of the visual field. We looked at the difference between attended and unattended conditions in several visual areas (V1, V2, VP, V4v and TEO) and for 3 different spatial separations $\left(2^{\circ}, 4^{\circ}\right.$ and $\left.7^{\circ}\right)$.

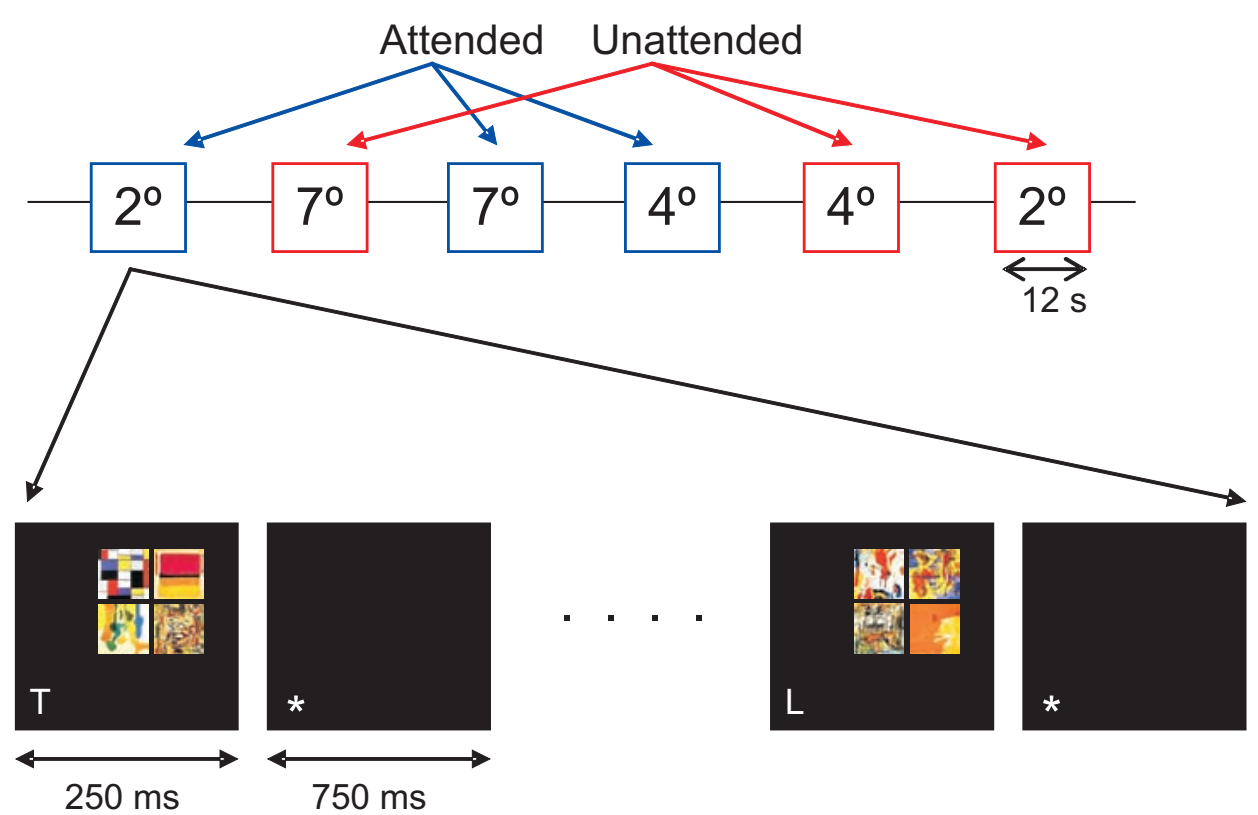

Figure 2.2: Experimental design. Each block lasted a total of 12 seconds and contained 12 picture presentations. Pictures were displayed on
screen for $250 \mathrm{~ms}$, followed by a blank screen with a fixation point for a duration of $750 \mathrm{~ms}$. Blocks were separated by a $18 \mathrm{~s}$ blank screen, a $3 \mathrm{~s}$
answer period (button-press), a $6 \mathrm{~s}$ instruction for the new block (target indication and location) and another $18 \mathrm{~s}$ blank screen.

The difference in BOLD-signal change between attended and unattended conditions was expected to be scaled with RF size and stimulus separation. We predicted the presence of this attention effect in an area on the basis of estimates of RF sizes for that area; the smallest spatial separation that leads to attention effects should match the size of the RF of that area at that eccentricity. Note that in the present study, stimuli were presented closer to the fovea (closest picture centered at $2.5^{\circ}$ eccentricity from the fixation point) than in the Kastner $\left(1998 ; 2001\right.$ a) studies (display of 4 pictures centered at $\sim 5.5^{\circ}$ eccentricity). Furthermore, we chose to present the attended stimulus always at the same position. Based on monkey electrophysiology (Boussaoud et al., 1991; Gattass et al., 1981; Gattass et 
al., 1988), and fMRI estimates of RF sizes in humans (Smith et al., 2001), RF size estimates at this eccentricity are smaller than $1^{\circ}$ for $\mathrm{V} 1,1-2^{\circ}$ for $\mathrm{V} 2, \sim 2^{\circ}$ for $\mathrm{VP}, 3-4^{\circ}$ for $\mathrm{V} 4 \mathrm{v}$ and 6- $7^{\circ}$ for TEO. This means that for the $2^{\circ}$ spatial separation condition in this study, the earliest area where to expect attention effects is $\mathrm{VP}$, for the $4^{\circ}$ condition it is $\mathrm{V} 4$, and for the $7^{\circ}$ condition it is TEO.

\section{Methods}

\section{Partic ipants}

Eight healthy volunteers (2 male, age 20-26 yrs) were paid for participation in this study, which was approved by an ethical committee. After explanation of the procedures, participants signed an informed consent form. None of the participants had any prior psychiatric or neurological history, were in good health and had normal or corrected to normal visual acuity.

\section{Visual task}

Display conditions were identical for attended and unattended conditions. When no pictures were displayed, there was a blank screen with a fixation cross. Trials consisted of displays of 4 pictures, which were randomly composed from a database of 135 complex, colorful pictures, each $1^{\circ}$ by $1^{\circ}$ in size. The displays were presented in the upper right quadrant of the visual field. During periods where pictures were present on the screen, an ' $L$ ' or a ' $T$ ' replaced the fixation cross. Participants performed one of two tasks. In the 'unattended' condition, participants had to fixate on the centre of the screen and had to count how often the fixation cross changed into a certain target letter, ignoring the pictures in the upper right quadrant. In the 'attend' condition, participants were instructed to fixate the centre of the screen but to covertly attend the picture that was presented closest to the fixation point and to count the occurrence of a target picture at that location. Target frequency randomly varied between 1 and 4 targets per block for both tasks. Picture displays covered an area of either $2^{\circ} \times 2^{\circ}, 4^{\circ} \times 4^{\circ}$ or $7^{\circ} \times 7^{\circ}$ of the visual field. The picture closest to the fixation point was always centered at $2.5^{\circ}$ from fixation point.

The order of the 6 blocks was randomized for each participant. Each block lasted 12 $\mathrm{s}$ and was preceded and followed by a blank screen for $16 \mathrm{~s}$ (see figure 2.2). In-between blocks, participants were asked to indicate their answer with a button press ( $3 \mathrm{~s}$ timeout) and received instructions for the next block (6 s). Stimuli had a duration of $250 \mathrm{~ms}$ and 
were presented at a rate of $1 \mathrm{~Hz}$. Each participant performed 3 runs, lasting about 7 minutes each, followed by an anatomical scan and the retinotopic mapping runs (see below for scan parameters).

\section{Data a cquisition}

Images were acquired on a 3T Siemens Trio Scanner (Siemens Medical Systems, Erlangen, Germany) using a standard head coil. Twenty-nine oblique axial slices (in-plane resolution: $3.5 \times 3.5 \mathrm{~mm}$, slice thickness: $4.5 \mathrm{~mm}$, interslice distance $0 \mathrm{~mm}$ ) covering the entire cortical volume were acquired using an echo planar imaging sequence (TR = $2000 \mathrm{~ms}$, TE $=29$ ms, matrix size: 64×64). There were 175 volumes per run, the first 4 of which were skipped due to the T1 saturation effect. Functional slices of each run were aligned to a high resolution anatomical dataset acquired after the functional runs (Magnetization-Prepared Rapid Acquisition Gradient Echo (MPRAGE); TR = 2300 ms, TE $=3.93 \mathrm{~ms}, 192$ slices).

The participants were placed comfortably in the scanner and their head was fixated with foam pads. Mounted on the head coil was a mirror through which they could see the stimuli projected on a screen placed outside the scanner. Stimulus presentation was synchronized with MR data acquisition by triggering the stimulus program with the first MR pulse.

\section{Retinotopic mapping}

For each participant retinotopic maps were created (Goebel et al., 2003; Sereno et al., 1995). Polar maps were acquired using a rotating, red-green blocked wedge of 33.75 degree polar angle covering eccentricities from 1 to 17 deg visual angle. Wedges were filled with a checkerboard pattern of red and green squares that reversed polarity 8 times per second and did a full rotation within 64 seconds. Thus each pixel in a circular field of view was activated every 64 seconds for a duration of 6 seconds. A functional run took 552 seconds, i.e. 8 cycles of rotating wedges plus 20 seconds lead in and lead out time. Scanning parameters: TR $=2000 \mathrm{~ms}$, TE $=30 \mathrm{~ms}, 30$ slices, voxel size $3.5 \times 3.5$ $\mathrm{mm}$, matrix size $64 \times 64$. Ventral visual areas V1, V2, VP and V4 were defined for each participant. Area TEO could not be reliably located for most participants by this method, but was defined as the area anterior to V4 that was activated reliably by the stimuli across participants, and was located at comparable Talairach coordinates as in the Kastner et al. (1998) study (see figure 2.3). Mean Talairach coordinates of each defined area are displayed in table 2.1. ROls were defined as those voxels of the mapped visual areas that showed significant activation in a contrast of all unattended conditions versus baseline (fixation), assuming that these activated areas include neurons with RF sensitive to the 


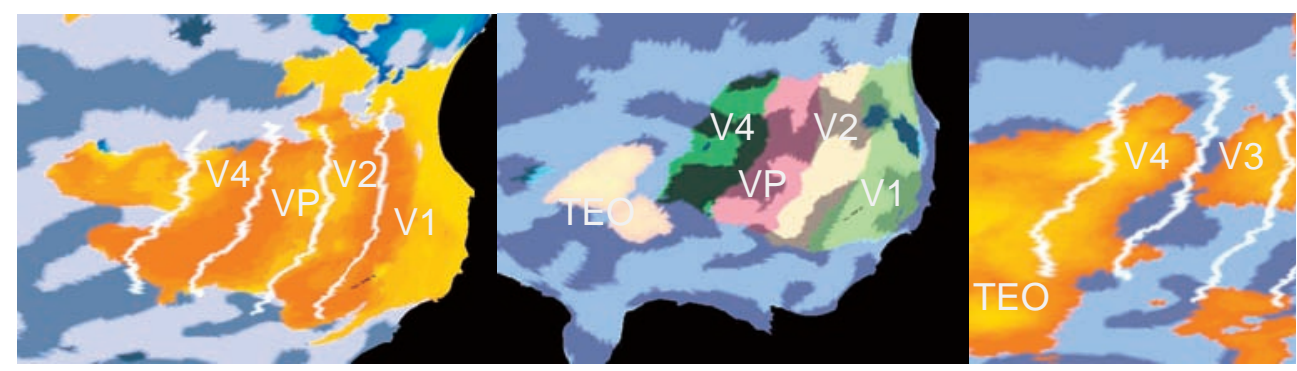

Figure 2.3: Regions of interest definition for one representative participant. The left graph shows the results of the polar mapping runs. The middle graph shows a flatmap of the visual areas defined by the polar mapping, including a projection of the activated part of area TEC. Shown in the right graph is the activation in 'unattended' conditions compared with baseline (fixation) to demonstrate which part of each visual area was taken as ROI across spatial separation conditions for this participant.

stimulated location. We thereby restricted data analysis to those voxels of the visual cortex in which the processing of our stimuli took place. The ROls within each area were identical for each spatial separation condition.

\begin{tabular}{rrrr}
\hline Area & $\mathbf{x}$ & $\mathbf{y}$ & $\mathbf{z}$ \\
\hline V1 & $-4 \pm 3$ & $-81 \pm 5$ & $-9 \pm 6$ \\
V2 & $-8 \pm 2$ & $-77 \pm 5$ & $-15 \pm 5$ \\
VP & $-16 \pm 2$ & $-75 \pm 5$ & $-16 \pm 4$ \\
V4 & $-22 \pm 2$ & $-72 \pm 6$ & $-15 \pm 5$ \\
TEO & $-26 \pm 2$ & $-53 \pm 9$ & $-12 \pm 4$ \\
\hline
\end{tabular}

Table 2.1: Mean talairach-coordinates of visual areas of interest $(\mathrm{mm} \pm \mathrm{SD}$

\section{Analysis}

Functional and anatomical images were analyzed with the BrainVoyager 2000 and BrainVoyager QX software packages (Brain Innovation, Maastricht, The Netherlands). Functional runs were corrected for slice scan time (using sinc interpolation) and for 3D head motion by using rigid body transformations. Linear trends and low-frequency nonlinear drifts $(<3 \mathrm{~Hz})$ were removed from the data. Functional data were then aligned to the anatomical scans and transformed into Talairach space. After definition of ROIs per participant (see "retinotopic mapping") , GLM beta weights were obtained per participant for all ROIs for each run, as an estimation of the contribution to the overall explained variance in the signal in the target area in a specific condition. These were then analyzed using a General Linear Model (repeated measures) with separate factors for spatial separation $\left(3 ; 2,4\right.$, and $7^{\circ}$ ), attention (2; attended and unattended) and visual area 
(5; V1, V2, VP, V4 and TEO ). Thereafter, paired samples T-tests were performed with both attention conditions as the paired variables, separately for each ROI and spatial separation. This enabled us to test the prediction that the smallest spatial separation that led to attention effects in an area was of the same magnitude as the estimated RF size for that area.

\section{Results}

The presentation of the stimuli activated areas V1, V2, VP, V4 and TEO across participants. Statistics are summarized in table 2.2. Figure 2.4 shows grouped beta weight differences between attended and unattended conditions for each area and for each spatial separation. The difference between attended and unattended conditions was significant $(F(1,22)=13.03, p<.01)$, with attended displays leading to higher beta weights, indicating that the BOLD-signal changes in this condition were higher than in unattended conditions. As can bee seen in figure 2.4, this difference increased as areas were located higher up the visual processing stream, which was reflected in a significant Attention $\times$ Area interaction $(F(4,88)=48.00, p<.001)$. Furthermore, there was a significant main effect of Spatial Separation $(F(2,44)=3.76, p<.05)$; overall activation was highest for the $4^{\circ}$ conditions, followed by the $7^{\circ}$ and then the $2^{\circ}$ conditions. We also observed a main effect of Area $(F(4,88)=59.95, p<.001)$; higher order areas tended to show higher beta weights than lower order areas. Finally, we observed a significant interaction between Spatial Separation and Area $(F(8,176)=5.01, p<.005)$.

Due to the main effect of Area and the interactions of this factor with Attention and Spatial Separation, we decided to look at each area separately by means of paired samples T-tests. These tests revealed that the difference in beta weights between attended

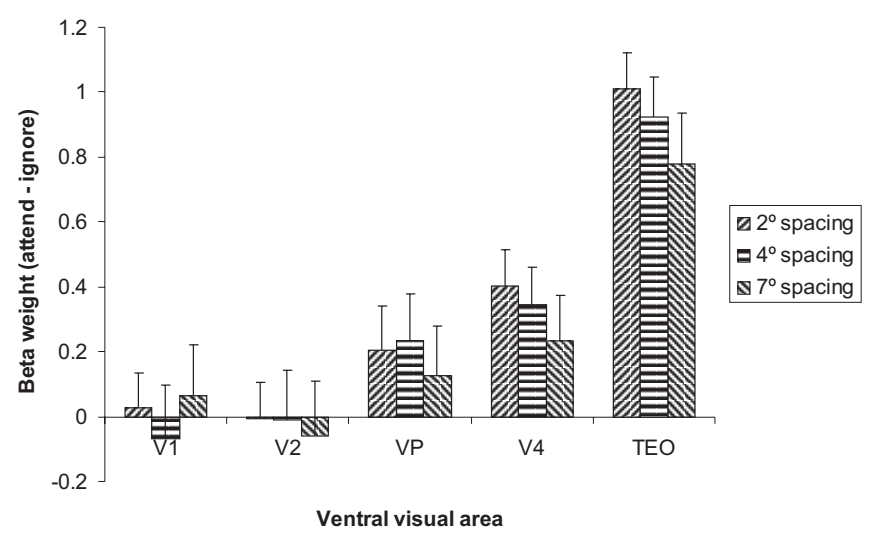


and unattended conditions did depend on RF size of an area. In area V4, this difference was significant for both $2^{\circ}$ and $4^{\circ}$ displays, (paired samples t-test, $t(22)>2.074, p<$ .005), but not for $7^{\circ}$ displays. Furthermore, GLM within-subjects difference contrasts show that the $7^{\circ}$ condition significantly differs from the $2^{\circ}$ and $4^{\circ}$ conditions: $F(1,22)=4.04$, $p<.05$ (one-tailed). In area TEO, all spatial separations led to a significant difference between attended and unattended conditions $(+(22)>2.074, p<.05)$. In none of the other areas the difference between attended and unattended conditions significantly deviated from zero.

\begin{tabular}{lrrl}
\hline Factor & F-value & dF & P - value \\
\hline Attention & 13.03 & 1,22 & $.002^{* *}$ \\
Spatial Separation & 3.76 & 2,44 & $.031^{*}$ \\
Area & 59.95 & 4,88 & $.000^{* * *}$ \\
Attention * Spatial Separation & .19 & 2,44 & .805 \\
Attention * Area & 48.00 & 4,88 & $.000^{* * *}$ \\
Spatial Separation *Area & 5.01 & 8,176 & $.000^{* * *}$ \\
Attention * Spatial Separation * Area & 1.16 & 8,176 & .333 \\
\hline
\end{tabular}

\section{Disc ussion}

In the current study, we aimed to support earlier findings by Kastner et al. (1998; 1999; 2001a), who found that BOLD signal change in the human brain during visual processing of pictures is decreased in conditions where competitive interactions take place among stimuli. Furthermore, we explicitly tested the more specific prediction of the biased competition theory that the difference between attended and unattended displays should be scaled to an area's RF size at a given eccentricity and the spacing between stimuli.

To investigate this, a direct comparison was made between attended and unattended presentations of stimuli with different spatial separations. BOLD signal changes were analyzed within participant's predefined visual areas of interest (contralateral ventral V1, V2, VP, V4, TEO). Our results support the biased competition model. The observed difference between attended and unattended displays was scaled to RF size of the reported areas; attention effects increased as RF size became larger. Furthermore, the smallest display that led to a difference between attended and unattended displays, matched the estimated size of the RF for that area. In lower order areas like V1 and V2, no attention effect was observed in any of the display spacings. Unexpectedly, we did not observe any significant attention effects in area VP. Possibly, our pictures were displayed at an 
eccentricity at which RF size was still too small to lead to competitive interactions for our smallest displays. Previously, attention effects have been observed in this area, but with stimuli presented at higher eccentricity (Kastner et al., 2001a), where RF sizes are larger. Further up the ventral stream, where RF size increases, attention effects were observed. Area $\mathrm{V} 4$ exhibited an effect for both $2^{\circ}$ and $4^{\circ}$ displays, which is in agreement with the estimated RF size of neurons in that area of about $4^{\circ}$ for that eccentricity (Boussaoud et al., 1991; Gattass et al., 1988; Smith et al., 2001). Similarly, in TEO all spatial separations led to the predicted attention effect, which is in agreement with the estimated RF size in TEO of $6 \sim 7^{\circ}$ (Boussaoud et al., 1991).

These data support the notion that the observed decrease in BOLD signal change in high competition conditions is a consequence of suppressive interactions between stimuli rather than a differential amount of transient stimulus onsets. Specifically, the data suggest that higher up the hierarchy of visual areas, competitive interactions between stimuli occur over larger distances, which is in agreement with the idea that these interactions are scaled to RF size. Correspondingly, the effect of attention on stimulus displays where competitive interactions take place also depends on the size of the RF of an area and the size of the stimulus display. The present findings further support the idea that biased competition, which has been studied predominantly with primate single-cell recordings, might also be at work in the human brain as a dominant stimulus selection mechanism.

\section{Acknowledgements}

This project was supported by NWO ASPASIA grant nr 015.001 .053 to B.M. Jansma (Schmitt) and by NWO grant nr 402-01-632 to R. Goebel .

The authors would like to thank Elia Formisano, Boukje Habets and Alard Roebroeck for valuable discussions in the preparation phase of this manuscript. Furthermore we would like to thank Henk Jansma for his help on segmenting our participants' brains, and Paul Gaalman for technical assistance during the fMRI measurements. 


\section{References}

Boussaoud, D., Desimone, R., and Ungerleider, L. G. (1991). Visual topography of area TEO in the macaque. J Comp Neurol 306, 554-575.

Broadbent, D. (1958). Perception and communication (London, Pergamon Press).

Chelazzi, L., Duncan, J., Miller, E. K., and Desimone, R. (1998). Responses of neurons in inferior temporal cortex during memory-guided visual search. Journal of Neurophysiology 80, 2918-2940.

Chelazzi, L., Miller, E. K., Duncan, J., and Desimone, R. (2001). Responses of Neurons in Macaque Area V4 During Memory-guided visual Search. Cerebral Cortex 11, $761-772$.

Desimone, R., and Duncan, J. (1995). Neural mechanisms of selective visual attention. Annu Rev Neurosci 18, 193-222.

Fox, P. T., and Raichle, M. E. (1985). Stimulus rate determines regional brain blood flow in striate cortex. Ann Neurol 17, 303-305.

Gattass, R., Gross, C. G., and Sandell, J. H. (1981). Visual topography of V2 in the macaque. J Comp Neurol 201, 519-539.

Gattass, R., Sousa, A. P., and Gross, C. G. (1988). Visuotopic organization and extent of V3 and V4 of the macaque. J Neurosci 8, 1831-1845.

Goebel, R., Muckli, L., and Kim, D.-S. (2003). The Visual System. In: G. Paxinos, and J. K. Mai, (Eds.), The Human Nervous System. New York, Academic Press.

Kastner, S., De Weerd, P., Desimone, R., and Ungerleider, L. (1998). Mechanisms of Directed Attention in the Human Extrastriate Cortex as Revealed by Functional MRI. Science 282, 108-111.

Kastner, S., Pinsk, M. A., De Weerd, P., Desimone, R., and Ungerleider, L. (1999). Increased Activity in Human Visual Cortex during Directed Attention in the Absence of Visual Stimulation. Neuron 22, 751-761.

Kastner, S., De Weerd, P., Pinsk, M. A., Elizondo, M. I., Desimone, R., and Ungerleider, L. (2001 a). Modulation of Sensory Suppression: Implications for Receptive Field Sizes in the Human Visual Cortex. Journal of Neurophysiology 86, 1398-1411.

Kastner, S., and Ungerleider, L. (2001 b). The neural basis of biased competition in human visual cortex. Neuropsychologia 39, 1263-1276.

Luck, S. J., Chelazzi, L., Hillyard, S. A., and Desimone, R. (1997). Neural mechanisms of spatial selective attention in areas $\mathrm{V} 1, \mathrm{~V} 2$, and $\mathrm{V} 4$ of macaque visual cortex. $J$ Neurophysiol 77, 24-42. 
Miller, E. K., Gochin, P. M., and Gross, C. G. (1993). Suppression of visual responses of neurons in inferior temporal cortex of the awake macaque by addition of a second stimulus. Brain Res 616, 25-29.

Moran, J., and Desimone, R. (1985). Selective attention gates visual processing in the extrastriate cortex. Science 229, 782-784.

Pinsk, M. A., Kastner, S., Desimone, R., and Ungerleider, L. (1999). An estimate of receptive field sizes in human visual cortex. Neurolmage 9, s885.

Price, C. J., Moore, C. J., and Frackowiak, R. S. (1996). The effect of varying stimulus rate and duration on brain activity during reading. Neuroimage 3, 40-52.

Recanzone, G. H., Wurtz, R. H., and Schwarz, U. (1997). Responses of MT and MST neurons to one and two moving objects in the receptive field. J Neurophysiol 78, 2904-2915.

Rees, G., Frackowiak, R., and Frith, C. (1997). Two modulatory effects of attention that mediate object categorization in human cortex. Science 275, 835-838.

Reynolds, J. H., Chelazzi, L., and Desimone, R. (1999). Competitive mechanisms subserve attention in macaque areas V2 and V4. J Neurosci 19, 1736-1753.

Schneider, W., Casey, B. J., and Noll, D. (1994). Functional MRI mapping of stimulus rate effects across visual processing stages. Human Brain Mapping 1, 117 - 133.

Sereno, M. I., Dale, A. M., Reppas, J. B., Kwong, K. K., Belliveau, J. W., Brady, T. J., Rosen, B. R., and Tootell, R. B. H. (1995). Borders of multiple visual areas in human revealed by functional magnetic resonance imaging. Science 268, 889-893.

Smith, A. T., Singh, K. D., Williams, A. L., and Greenlee, M. W. (2001). Estimating receptive field size from fMRI data in human striate and extrastriate visual cortex. Cereb Cortex 11, 1182-1190.

Treisman, A. M. (1969). Strategies and models of selective attention. Psychol Rev 76, 282-299. 


\section{○}

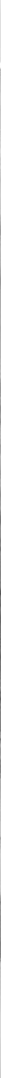




\section{The time course of competitive and biasing mechanisms in human visual cortex}

According to the influential 'biased competition' framework, stimuli compete for neural representation. This competition can be biased in favour of a stimulus through bottom-up and top-down mechanisms. Support for this framework comes from monkey neurophysiology and, more recently, human neuroimaging studies. The current study exploited the high temporal resolution of event-related potentials to explore whether a time window exists in which competitive stimulus interactions can be observed in the absence of an attentional bias. Four colourful, abstract pictures covering either $2^{\circ}, 4^{\circ}$ or $7^{\circ}$ of the visual field were presented simultaneously at peripheral locations while attention was directed at one of the pictures or at the fixation point (i.e. away from the stimuli). The posterior P1 component was found to be modulated by the spatial separation between the stimuli, but not by attention. The subsequent frontal $\mathrm{N} 1$ component was modulated by both spatial separation and attention, and was followed by a posterior $\mathrm{N} 1$ that revealed an interaction between spatial separation and attention. These results imply that simultaneously presented stimuli compete for neural representation, and that this competition is biased through top-down mechanisms only after a first rudimentary analysis of the scene has taken place. 


\section{Introduction}

An everyday visual scene is cluttered with objects. Since our brain cannot completely process all these objects at a given time (e.g. Broadbent, 1958; Treisman, 1969), stimuli are said to compete for neural representation. According to the 'biased competition' approach, this competition can be biased in favour of stimuli with salient features (bottomup mechanisms) or stimuli that are behaviourally relevant to us (top-down mechanisms) (for an overview, see Desimone \& Duncan, 1995; Kastner \& Ungerleider, 2001 b). The processing of these biased stimuli is enhanced compared to that of others, enabling them to influence behaviour and cognitive states. This influential approach has received support from neurophysiological studies in animals (e.g. Chelazzi et al., 1998; Luck et al., 1997; Moran \& Desimone, 1985; Reynolds et al., 1999) and, more recently, from human neuroimaging studies (Beck \& Kastner, 2005; Bles et al., 2006; Kastner et al., 1998; Kastner et al., 1999; Kastner et al., 2001 a; Pinsk et al., 1999). The aim of the present study was to apply a 'biased competition' paradigm in an event-related potential (ERP) study. Due to their high temporal resolution, ERPs can yield important insights in the temporal aspects of competitive processes between stimuli.

Results from single-cell recordings in extrastriate cortex indicate that whenever multiple stimuli are presented within the receptive field (RF) of a neuron in the absence of attention, these stimuli compete for control over that neuron's firing rate. When, for example, an effective and an ineffective stimulus are presented together within the RF of a macaque V2 or V4 neuron, the cell responds with a firing rate that is a weighted average of the firing rates evoked by the single stimuli (Chelazzi et al., 1998; Chelazzi et al., 2001 ; Luck et al., 1997; Miller et al., 1993; Moran \& Desimone, 1985; Recanzone et al., 1997; Reynolds et al., 1999). Thus, instead of being processed independently, multiple stimuli within the RF of a neuron can be seen as mutually suppressing each other's representation and competing for neural resources. Importantly, when one of the stimuli is attended, competition is biased in favour of this stimulus. The processing of this stimulus then gains precedence over that of others, and the neuron's firing rate will reflect the presence of the attended stimulus, but not that of the distractors. So if the effective stimulus is attended to, the neuron's firing rate increases to a level that is approximately the same as when the effective stimulus is presented alone. Likewise, if the ineffective stimulus is attended to, firing rate decreases to the level that can be observed when the ineffective stimulus is presented alone (Chelazzi et al., 1998; Chelazzi et al., 2001; Luck et al., 1997; Moran \& Desimone, 1985; Reynolds et al., 1999).

More recently, support for the biased competition account has been obtained in human participants where competitive interactions between stimuli were observed in extrastriate visual cortex with BOLD-imaging fMRI (Beck \& Kastner, 2005; Bles et al., 2006; Kastner et al., 1998; 1999; 2001a). For example, Kastner et al. (1998; 2001 a) showed 
participants displays of 4 complex, colourful, visual stimuli which were presented either simultaneously or sequentially. In the simultaneous condition stimuli could compete for neural representation since visual areas have to process the information of several stimuli at the same time. In the sequential condition there was only one stimulus presented at a time, preventing competition between stimuli. The BOLD response in participants' visual areas V2/VP, V4 and TEO was decreased when stimuli were presented simultaneously as compared to the signal obtained when stimuli were presented sequentially. The authors interpreted these findings as evidence that during simultaneous stimulation the neural representations of the different stimuli interact in a suppressive way and that no such competition takes place during sequential stimulation (Kastner et al., 1998; 2001a). Moreover, suppression effects were not observed when participants attended one of the stimuli, suggesting that attention biased the competition in favour of the attended item, relieving it from the suppressive effects of the surrounding stimuli (Kastner et al., 1998; 1999).

As visual areas are located further up the cortical visual processing stream, their average RF size increases. A consequence of this characteristic is that on average more stimuli will be present within the RFs of higher order areas compared to lower order areas, given an identical display. This leads to the prediction that stronger competitive interactions between stimuli should be observed in higher order areas, since neurons in these areas have larger RFs. Indeed, Kastner et al. $(1998$; 1999) observed that the competitive interactions were not of the same magnitude in all visual areas, but rather increased as areas were located higher up the visual stream. Furthermore, some studies explicitly tested the prediction that competitive interactions between stimuli are dependent on RF size (Bles et al., 2006; Kastner et al., 2001 a; Pinsk et al., 1999). In these studies the spatial separation between the stimuli in the display was systematically varied. By placing them further apart, less stimuli fell within the same RF (see figure 2.1) and therefore weaker competitive interactions were expected. Indeed, within a given area the magnitude of the suppressive interactions decreased with increasing stimulus separation. In addition, suppressive interactions increased from lower to higher visual areas. In line with the biased competition theory, this suggests that the magnitude of the competitive interaction effect scales with RF size of an area and the spatial separation between stimuli.

Although it is clear that attention improves processing of stimuli at attended locations and that it can resolve competition between simultaneously presented stimuli, a question that remains is exactly when attention influences competitive processes in the visual processing stream. In this respect, the high temporal resolution of the human ERP can yield important information.

In human psychophysiological studies, attention has been studied in the context of spatial and feature selection. In general, attention effects are observed at the early P1 and $\mathrm{Nl}$ components of the ERP, whereas the even earlier $\mathrm{Cl}$ component is notoriously 
unaffected by attention. In spatial attention paradigms, the amplitude of the $\mathrm{P} 1$ and $\mathrm{N} 1$ components is higher for stimuli at attended locations than at ignored ones, suggesting an overall larger neural responses to stimuli at attended locations (see Hillyard \& AnlloVento, 1998; Hillyard et al., 1998 for a review). Studies that measured attention effects with FMRI and ERP in the same participants, show that the origin of the P1 component lies in extrastriate cortex (Di Russo et al., 2003; Martinez et al., 1999; 2001; Noesselt et al., 2002). Interestingly, even though fMRI results revealed increased activation in striate cortex in these studies, the $\mathrm{Vl}$-generated $\mathrm{Cl}$ component was unaffected by the attention manipulations. As an explanation for this discrepancy between fMRI and ERP pattern, it was suggested that the primary visual cortex effects observed with FMRI reflect a later re-entrant feedback of information from extrastriate visual areas or a biasing signal from higher-order areas that leaves the initial, stimulus-driven feed-forward sweep of activation unaffected. Consistent with this idea, dipole localisation of a later negativity (around 160 - $230 \mathrm{~ms}$ ) that was modulated by attention, indicated the same neural generators in the primary visual cortex (Di Russo et al., 2003; Martinez et al., 2001; Noesselt et al., 2002). Although spatial attention paradigms have shed light on the ERP components that are influenced by spatial attention, they usually employ single stimuli at either the attended or the unattended location, which makes these designs not directly suitable to investigate the sensory competitive processes taking place when multiple stimuli are presented.

Only a few ERP studies have employed multiple stimulus displays, mainly in the context of a visual search paradigm (see Hopf et al. (2005) for a review). The primary research interest of these studies was to investigate how attention enhances stimulus processing or selects a target from a set of stimuli. They did not focus on sensory suppression directly. Typically, in these paradigms a target is presented at an unknown location and is flanked with distractor items. ERPs contralateral to the target location exhibit an increased N2pc component, which has been associated with attentional focusing on the target stimulus (Hopf et al., 2005; Luck \& Hillyard, 1994; Woodman \& Luck, 1999). The magnitude of the N2pc effect is enhanced when the amount of similarity between targets and distractors is increased, indicating that this component is involved in the suppression of distractors, which is needed more in case of similarity between target and distractor (Hopf et al., 34 2002).

Jacques \& Rossion $(2004$; 2006a; 2006b) investigated competitive effects between stimuli, in this case pictures of faces. They presented a face or a control stimulus (scrambled face) to which a face stimulus was added. The task of the participants was to detect orientation, location or luminance changes in the second face. The rationale behind this study was that if the initially presented stimulus was a face, the representation of this face is still present in face processing areas when the second face is displayed. Consequently, the neural representation of the two stimuli engage in competitive interactions for representation, leading to a reduced neural activity (i.e. ERP amplitude reduction).The 
ERP component of interest was the N170, which is consistently observed in response to the presentation of face stimuli (Bentin et al., 1996) and is thought to reflect the high-level face processing occurring throughout higher-order visual areas (Henson et al., 2003). Indeed, they observed that the N170 in response to a face stimulus was reduced when a face stimulus was already present in the display, compared to the control stimulus. These results were in compliance with models of visual processing that assume that stimuli compete for neural representation, such as the biased competition model. However, the biased competition account makes specific predictions about competition effects when attention is directed away from the competing stimuli. These studies did not include such a manipulation.

The goal of the current study was to directly address the effects of competition between simultaneously presented stimuli on the ERP, both when attention was directed towards one of these stimuli and when it was directed away from them. To achieve this, the design employed by Bles et al. (2006) was modified for ERP purposes. In this design, four colourful, abstract stimuli were always simultaneously presented, and the amount of competition was defined as the difference between attended and unattended displays. By systematically varying the spatial separation between stimuli $\left(2^{\circ}, 4^{\circ}\right.$, or $\left.7^{\circ}\right)$, the relative overlap of stimuli within RFs - and hence the amount of competition - was parametrically manipulated within visual areas. The four stimuli were always presented in the upper right corner of the visual field, while participants were either performing a line comparison task at fixation (unattended pictures condition), or carried out a match-to-sample task with one of the colourful stimuli (attended picture condition). Stimulus displays, arranged in a $2 \times 2$ array, covered a $2^{\circ} \times 2^{\circ}, 4^{\circ} \times 4^{\circ}$, or $7^{\circ} \times 7^{\circ}$ area of the visual field, the stimulus closest to the fixation point always being centred at $2.5^{\circ}$ degrees from the fixation point. As observed previously, in the unattended picture condition, stimuli were expected to interact in a mutually suppressive way. Attending one of the stimuli should bias competition in favour of this stimulus, and relieve it of the suppressive influences of the distractors. Hence, if we define the amount of competition as the difference between attended and unattended picture displays (see Bles et al., 2006), this difference should increase with smaller display spacing, or with increasing RF sizes along the visual pathway (approx. $<1^{\circ}$ in $\mathrm{V} 1,3^{\circ}$ $4^{\circ}$ in V4, and $6^{\circ}-7^{\circ}$ in TEO at this eccentricity, see Bles et al., 2006; Boussaoud et al., 1991; Gattass et al., 1981; 1988; Smith et al., 2001).

Due to the intrinsic low spatial resolution, ERPs measured at the scalp reflect the integrated firing of many cortical areas. Although we could not spatially disentangle the responses of extrastriate visual areas in this study, the ERP components reflect neural activity across these regions. Therefore, we expected the amplitude difference between attended and unattended displays to be largest for the $2^{\circ}$ displays (since these should lead to competition in most areas) followed by the $4^{\circ}$ and $7^{\circ}$ displays (since these should lead to competition only in areas beyond $\mathrm{V} 4$ ). These effects were expected on the early P1 and 
N1 components, and possibly later components like the P2, N2 and P3. In addition, we were interested whether these components of interest distinguished between bottom-up competitive stimulus interactions (reflected in the $\mathrm{Cl}$ and $\mathrm{Pl}$ comoponents) and top-down biasing signals (reflected in the P1, N1, N2pc and P3 components).

\section{Methods}

\section{Partic ipants}

Twenty-one healthy volunteers (four males, mean age 23.6 years, two left-handed) participated in this study. Participants had no prior psychiatric or neurological impairments and had normal or corrected-to-normal visual acuity. After explanation of the experimental procedures, participants gave informed consent. Participants were paid for participation and ethical approval was obtained from the ethical committee of the Faculty of Psychology.

\section{Material and Task}

Display conditions were identical for attended and unattended conditions. The Stimuli were randomly drawn from a database of 175 complex, colourful pictures, each $0.8^{\circ}$ by $0.8^{\circ}$ in size (see Bles et al. (2006)). When a trial started, 4 pictures were displayed in a $2 \times 2$ array, covering an area of either $2^{\circ} \times 2^{\circ}, 4^{\circ} \times 4^{\circ}$ or $7^{\circ} \times 7^{\circ}$ of the visual field. The picture closest to the fixation point was always centred at $2.5^{\circ}$ from the fixation point. At the moment of picture display, the fixation cross was replaced by two bars to allow for the control line-comparison task. The bars were $.2^{\circ}$ high, 1 to 4 pixels wide, and were presented $.15^{\circ}$ degrees above and below the centre of the screen. On target trials they were of the same width, on non-target trials they differed in width by one pixel. A trial started with a black screen with a fixation cross. After a period of $1400-1900 \mathrm{~ms}$, the 4 pictures were presented for $250 \mathrm{~ms}$ in the upper right quadrant of the visual field together with the 2 bars around the fixation cross.

Participants performed one of two tasks. In the 'unattended' condition, participants had to fixate on the centre of the screen and had to press a response button with their right index finger whenever the lines were of the same width. No response was required for different line width. Meanwhile, they ignored the pictures in the upper right quadrant. In the 'attended' condition, participants were instructed to fixate the centre of the screen but to covertly attend the picture that was presented closest to the fixation point and to press a response button with their right index finger when a target picture occurred at that location, no response was required for non-targets. Targets randomly occurred in 10\% 
of trials for both tasks. The six conditions ( 3 degree conditions: $2^{\circ}, 4^{\circ}, 7^{\circ}$ times 2 tasks) were presented in blocks, each repeated three times in a randomized order. Each block contained 60 trials, including six target trials, which were excluded from analysis.

\section{Procedure}

Participants were tested individually while seated in a electrically shielded, soundproof chamber in front of a computer screen. They were instructed to rest their arms and hands on the table and were instructed not to speak, blink or move their eyes while the pictures were on the screen. Furthermore, they rested their heads in a chin-rest to prevent head movements and to assure a fixed distance between the eyes and the monitors so that the size of each display on the participants' retina was controlled for. Before the experiment commenced, participants received task instructions and performed two practice blocks to check whether instructions were clear and to monitor eye movements.

\section{EEG recording}

The electroencephalogram (EEG) was measured using 29 tin electrodes mounted in an elastic cap (QuickCap, Neuroscan Inc. Tm). The electrodes were positioned according to the extended international 10-20 system (Nuwer, 1998). Electrodes Fz, F3, F4, F7, F8, FCz, FC3, FC4, FP1 , FP2, AFz, T3, T4, T5, T6, TP7, TP8, Cz, C3, C4, CPz, CP3, CP4, Pz, $\mathrm{P} 3, \mathrm{P} 4, \mathrm{Oz}, \mathrm{O} 1, \mathrm{O} 2$ were measured as active leads. Scalp sites were online referenced to an electrode placed at the left mastoid (A1). The signal of an additional electrode placed at the right mastoid (A2), referenced to $A 1$, was later used for offline re-referencing of the signal to the average of $A 1 / A 2$. Vertical eye movements and blinks were measured with a bipolar montage placed above and below the left upper and lower orbital ridge. Horizontal eye movements were recorded with a bipolar montage placed at the left and right canthi. All electrode impedances were kept below $5 \mathrm{KOhm}$. The EEG was amplified (Synamp, Neuroscan Inc.) with a $.05-40 \mathrm{~Hz}$ band pass (notch filter at $50 \mathrm{~Hz}$ ) and sampled at $4 \mathrm{~ms}$ intervals $(250 \mathrm{~Hz})$ and stored to disk. Epochs of $800 \mathrm{~ms}$ were created, time-locked to the presentation of the visual stimuli on the screen. Epochs included a $200 \mathrm{~ms}$ prestimulus baseline-correction interval and they were $30 \mathrm{~Hz}$ low pass filtered (Butterworth, zero phase, $24 \mathrm{~dB} / \mathrm{oct}$ ). After removal of eye blinks and other artefacts, the data of five participants were discarded from further analysis due to an artefact rejection rate higher than $25 \%$ in at least one of the conditions. ERPs were calculated by averaging the time courses of the artefact-free trials (on average $90 \%$ of the trials, no difference between conditions) separately for each participant and each condition. 


\section{Results}

The stimuli elicited a clear ERP in all participants. The grand average ERP (see figures 3.1 and 3.2) showed a clearly visible P1 component at posterior scalp sites, with a maximum around $130 \mathrm{~ms}$ post-stimulus. This was followed by an $\mathrm{N} 1$ component peaking around $150 \mathrm{~ms}$ at anterior sites and slightly later, at $180 \mathrm{~ms}$ at posterior sites. Subsequently, the scalp voltages deflected positively again towards a P2 component with a maximum around $220 \mathrm{~ms}$, followed by a late negativity throughout scalp regions. Mean area amplitudes were calculated in appropriate time windows around these components for each condition for each participant, and were entered in a repeated measures General Linear Model (GLM) analysis with spatial separation (3 levels: $2^{\circ}, 4^{\circ}$ and $7^{\circ}$ ), laterality (3 levels: left ( $\mathrm{F} 3$ to $\mathrm{O} 1)$, middle ( $\mathrm{Fz}$ to $\mathrm{Oz}$ ), right ( $\mathrm{F} 4$ to $\mathrm{O} 2)$ ) and anterior-posterior (6 levels: F, FC, C, CP, P and $O$ lines) as factors. All reported p-values of tests with more than one degree of freedom in the numerator were Greenhouse-Geisser corrected to adjust for violations of the sphericity assumption (Greenhouse \& Geisser, 1959).

\section{0 - 150 ms time window (posterior P1)}

The posterior P1 component had a maximum around 130 ms. The GLM with factors attention (2), spatial separation (3), anterior-posterior (6) and laterality (3) revealed that the $\mathrm{Pl}$ component had a clear posterior distribution (see figure 3.1 and 3.2), reflected in a significant main effect of the anterior-posterior factor $(F(5,75)=22.13, p<.001)$. Hence, statistics in this time-window are only reported for posterior electrode sites in the $P$ and $O$ lines.

No attention effects were observed in this time window at these sites $(F(1,15)<1.7$, $\mathrm{p}>$.2). A main effect of spatial separation was observed in this time-window at the $P$ line $(F(2,30)=3.75, p<.05)$ (see figure 3.2). Pairwise comparisons revealed a significant difference between the $2^{\circ}$ and $7^{\circ}$ conditions $(p=.041)$, and a marginally significant difference between the $2^{\circ}$ and $4^{\circ}$ conditions $(p=.08)$. Spatial separation interacted with the lateralisation factor $(F(4,60)>9.69, p=.001)$. The most positive amplitudes were observed in the $7^{\circ}$ condition, followed by the $4^{\circ}$ and $2^{\circ}$ conditions, but this effect was slightly larger at right-hemispheric scalp sites (see figure 3.2, top topographic plot). No effects of spatial separation were observed at the $\bigcirc$ line $(F(2,30)=1.02, p>.3)$. 
A) Attention
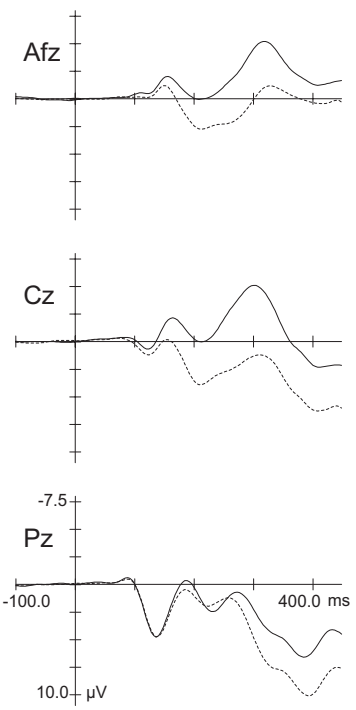

Attended

Unattended
B) Spatial separation
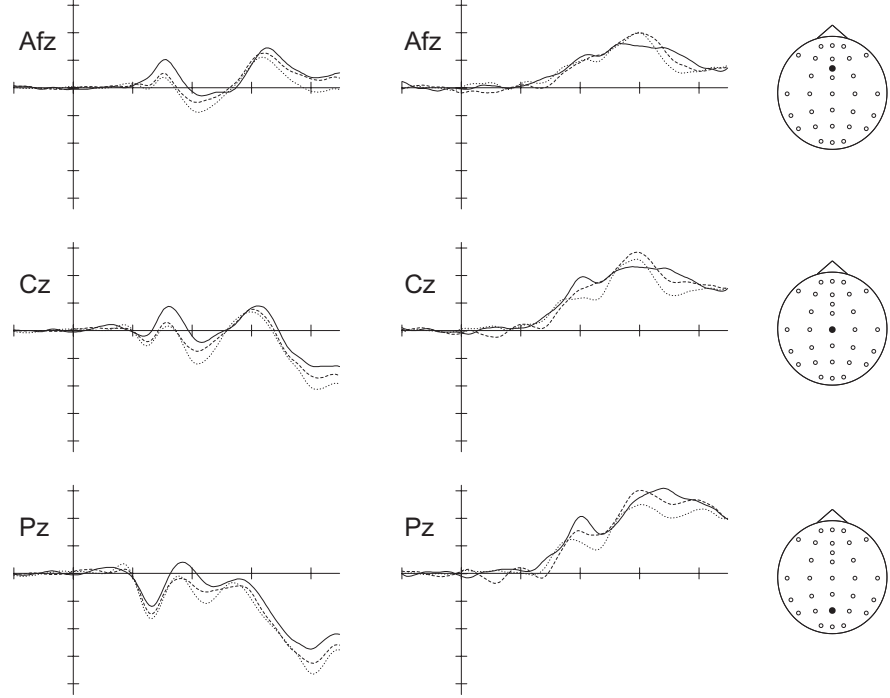

Attended $2^{\circ}$ - Unattended $2^{\circ}$

Attended $4^{\circ}$ - Unattended $4^{\circ}$

Attended $7^{\circ}$ - Unattended $7^{\circ}$

\section{0 - 180 ms time window (frontal N1)}

The anterior N1 component was maximal around $160 \mathrm{~ms}$. The GLM with factors attention (2), spatial separation (3), anterior-posterior (6) and laterality (3) revealed that it had a clear anterior distribution, reflected in a significant main effect of the anteriorposterior factor $(F(5,75)=10.48, p<.0054)$. Hence, subsequent statistics in this timewindow are only reported for anterior electrode sites in the F, FC and C lines.

Attention effects were observed in this time window at these sites $(F(1,15)>35.53$, $p$ $<.001$ ) (see figure 3.1). Attended conditions led to more negative amplitudes in this time window than unattended conditions. We also observed a significant main effect of spatial separation at each of these electrode lines $(F(2,30)>18.71, p<.001)$ (see figure 3.2). Pairwise comparisons revealed that each spatial separation significantly differed from the others (all p-values $<$.05). Most negative voltage amplitudes were observed in the $2^{\circ}$ condition, followed by the $4^{\circ}$ and $7^{\circ}$ conditions, respectively (see figure $3.2 \mathrm{a}$ ). 


\section{0 - 230 ms time wind ow (posterior N1)}

The posterior $\mathrm{N} 1$ component peaked around $185 \mathrm{~ms}$. Due to its salient posterior distribution, statistics in this time-window are only reported for posterior electrode sites in the $\mathrm{CP}, \mathrm{P}$ and $\mathrm{O}$ lines. Attention effects were observed in this time window at the $C P$ and $P$ lines $(F(1,15)<41.04, p .001)$. Attended conditions led to more negative amplitudes in this time window than unattended conditions. This effect was most apparent

A) Attention

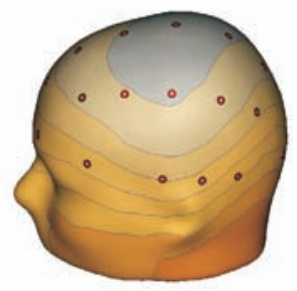

$144 \mathrm{~ms}$

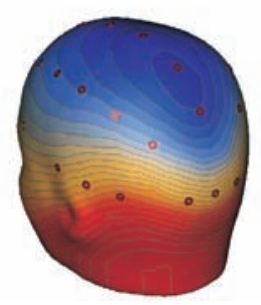

$196 \mathrm{~ms}$

40
B) Spatial separation

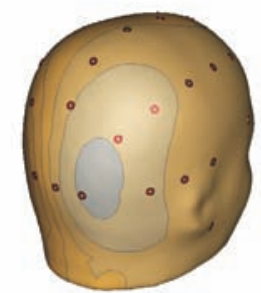

$120 \mathrm{~ms}$

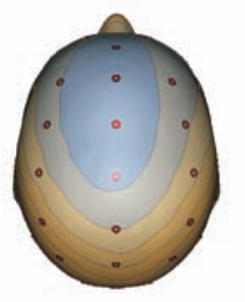

$160 \mathrm{~ms}$

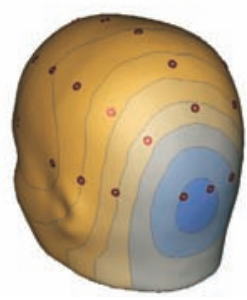

$292 \mathrm{~ms}$

$2^{\circ}-7^{\circ}$
C) Interaction

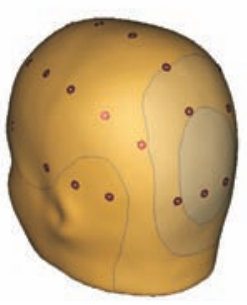

$120 \mathrm{~ms}$

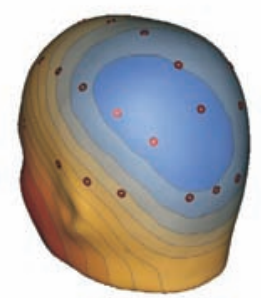

$196 \mathrm{~ms}$

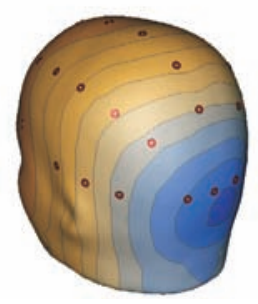

$292 \mathrm{~ms}$

(Attended $2^{\circ}$ - Unattended $2^{\circ}$ ) (Attended $7^{\circ}$ - Unattended $7^{\circ}$ )

Attended - Unattended

$2^{\circ}-7^{\circ}$ 
at midline electrodes (see figure 3.2a): at the CP and $\mathrm{P}$ lines, attention interacted with laterality $(F(2,30)>9.86, p<.001)$. The largest attention effect was observed at the midline electrodes, followed by the left and right electrodes, respectively. No main effect of attention was apparent at the $\bigcirc$ line $(F(1,15)=.12, p>.5)$

A main effect of spatial separation was observed at the $C P, P$ and $O$ lines $(F(2,30)$ $=8.18, \mathrm{p}<.005$ ) (see figure 3.2b). Pairwise comparisons revealed that each condition significantly differed from each other $(p<.05)$, except for the $4^{\circ}$ and $7^{\circ}$ conditions at the $\bigcirc$ line $(p>.1)$. Most negative amplitudes were observed for the $2^{\circ}$ conditions, followed by the $4^{\circ}$ and $7^{\circ}$ conditions, respectively. This effect was effect was most clear at midline electrodes: at all lines, there was a significant interaction between spatial separation and lateralization $(F(4,60)>3.2, p<.05)$ : in the $4^{\circ}$ and $7^{\circ}$ conditions. The highest amplitudes were observed at the central electrode, followed by the corresponding left and right electrode in each line, respectively.

At the $P$ and $O$ lines, there was a significant interaction between attention and spatial separation $(F(2,30)>3.85, p<.04)$ (see 3.2c): pairwise comparisons revealed that at both lines, the difference between attended and unattended displays was significant between the $2^{\circ}$ and $7^{\circ}$ conditions $(p<.05)$. At the $\bigcirc$-line, the difference between $2^{\circ}$ and $4^{\circ}$ was marginally significant $(p=.07)$. The largest difference was observed in the $2^{\circ}$ condition, followed by the $4^{\circ}$ and $7^{\circ}$ conditions, respectively.

\section{0 - 250 ms time window (P2)}

The $\mathrm{N} 1$ was followed by a P2 component, peaking around $220 \mathrm{~ms}$ at anterior sites and $230 \mathrm{~ms}$ at posterior sites. Since no differences in attention or spatial separation were observed through visual inspection between anterior and posterior sites, they are analysed as one time window. The GLM with factors attention (2), spatial separation (3), anterior-posterior (6) and laterality (3) revealed a main effect of both attention ( $F(1$, $15)=61.11, p<.001)$ and spatial separation $(F(2,30)=10.27, p<.001)$, but no interaction between the these factors $(F(2,30)=2.71, p>.05)$. Due to significant 3 -way interactions between attention, anterior-posterior and laterality $(F(10,150)=6.26$, $p<.005)$ and spatial separation, anterior-posterior and laterality $(F(20,300)=2.38$, $p$ $<.05$, we decided to look at each level of the anterior-posterior factor separately.

Significant effects of attention were observed at the F, FC, C, CP and P lines ( F (1, 15) $>29.79, p<.001)$ : unattended conditions led to more positive amplitudes than attended conditions. Similarly, a significant main effect of spatial separation was observed at the F, FC, C, CP and $P$ lines $(F(2,30)>7.94, p<.005)$ : most positive amplitudes were observed in the $7^{\circ}$ conditions, followed by the $4^{\circ}$ and $2^{\circ}$ conditions, respectively. Pairwise comparisons at each line revealed that the difference between the $2^{\circ}$ and $7^{\circ}$ conditions $(p<.01)$ and between the $4^{\circ}$ and $7^{\circ}$ conditions $(p<.05)$ was significant across the 
scalp. The difference between $2^{\circ}$ and $4^{\circ}$ approached significance at the P-line $(p=.08)$. No interactions between attention and spatial separation were observed at any of the electrode lines (all p-values $>$.08). In addition, attention effects were most apparent at midline electrode sites (see figure 3.2a): at the F, FC, C, CP and P lines, there was a significant interaction between attention and laterality $(F(2,30)>6.64, p<.02)$.

\section{0 - 310 ms time window (late negativity, early part)}

The P2 was followed by a negative-going component across the scalp that peaked around 310 ms but was present until the end of the epoch at $450 \mathrm{~ms}$. Since differences in spatial separation effects were observed through visual inspection of the waves between the early and the late part of this component, this component was split in two time windows for analysis. The GLM with factors attention (2), spatial separation (3), anterior-posterior (6) and laterality (3) revealed that it had a clear anterior distribution (see figure 3.1 a), reflected in a significant main effect of attention $(F(1,15)=80.45, p<.001)$. No main effect of spatial separation was observed in this time window $(F(2,30)=1.54, p>.2)$, but spatial separation strongly interacted with the anterior-posterior factor $(F(10,150)=$ $9.45, \mathrm{p}<.001)$.

Separate GLMs at each electrode line revealed that, whereas attended conditions led to more negative amplitudes than unattended conditions across the $\operatorname{scalp}(F(1,15)>5.25$, $p<.04)$, effects of spatial separation were only present at the $P(F(2,30)=5.61, p<$ $.015)$ and $O(F(2,30)=16.61, p<.001)$ lines: most negative amplitudes were observed in the $2^{\circ}$ conditions, followed by $4^{\circ}$ and $7^{\circ}$, respectively. Pairwise comparisons revealed that at both lines the $2^{\circ}$ conditions significantly differed from the $4^{\circ}$ and $7^{\circ}$ conditions ( $p$ $<.02)$, but there was no significant difference between the $4^{\circ}$ and $7^{\circ}$ conditions ( $p>$ .1). The attention effect in this time window was most apparent at midline electrodes (see figure 3.2a): a significant interaction between attention and laterality was observed at the $P$ line $(F(2,30)=15.56, p<.001)$. At the $O$ line, spatial separation interacted with attention $(F(2,30)=3.96, p<.04)$ : the difference between attended and unattended displays was largest for $2^{\circ}$ and $4^{\circ}$ conditions, followed by the $7^{\circ}$ condition.

\section{0-450 ms time window (late negativity, la te part)}

The GLM with factors attention (2), spatial separation (3), anterior-posterior (6) and laterality (3) revealed a significant main effect of attention $(F(1,15)=55.84, p<.001)$ : attended conditions led to more negative mean amplitudes than unattended conditions. In addition, a significant main effect of spatial separation was observed $(F(2,30)=10.18$, $p<.001)$ : most negative amplitudes were observed in the $2^{\circ}$ condition, followed by the $4^{\circ}$ and $7^{\circ}$ conditions, respectively. Pairwise comparisons revealed a significant difference 
between each of the spatial separations $(p<.05)$. No interaction between attention and spatial separation was observed, nor were there any 3-way or 4-way interactions involving both these factors (all p-values $>.2$ ).

Separate GLMs at each electrode line confirmed that attended conditions led to more negative amplitudes than unattended conditions across the $\operatorname{scalp}(F(1,15)>22.27, p<$ $.001)$. In addition, the spatial separation effect was present at each electrode line $(F(2$, 30) $>4.51, p<.03)$. No interactions between spatial separation and attention were observed at any of the electrode lines (all p-values > .4).

Attention effects were slightly larger at midline scalp sites than at lateralised sites (see figure 3.2a), apparent in a significant interaction between attention and lateralization was observed at the $F C, C, C P$ and $P$ lines $(F(2,30)>6.09, p<.01)$. Finally, the effect of spatial separation was left-lateralized: a significant interaction between spatial separation and lateralization was observed at the $\mathrm{CP}$ and $\mathrm{P}$ lines $(\mathrm{F}(4,60)>4.27, \mathrm{p}<.01)$.

\section{Disc ussion}

The goal of this study was to explore the time course of sensory suppression and attentional biasing mechanisms in human visual cortex in light of a biased competition framework. Participants viewed displays of four simultaneously presented stimuli in the periphery, which were spaced either $2^{\circ}, 4^{\circ}$ or $7^{\circ}$ apart. In addition, one of the pictures in the display was attended, or participants performed a task at the fixation point (pictures unattended). Pictures were expected to exhibit mutual suppression effects in unattended conditions, which should scale negatively with spatial separation (i.e. larger suppression for smaller displays). In addition, we expected to find neural correlates of a top-down biasing signal in attended conditions. This signal too should scale negatively with spatial separation (i.e. stronger bias effect in conditions with high amounts of competitions (small spatial separations)).

The earliest effects of spatial separation were observed at the posterior $\mathrm{Pl}$ component. In line with accounts that would predict that stimuli compete for neural resources, smallest $\mathrm{P} 1$ amplitudes were observed in the $2^{\circ}$ conditions, followed by the $4^{\circ}$ and $7^{\circ}$ conditions, respectively. These results support the notion that competitive effects between stimuli are reflected in lower ERP amplitudes, as has been observed with face stimuli (Jacques \& Rossion, 2004; 2006a; 2006b). However, participants in previous studies always attended one of the face stimuli. A competitive advantage for the unattended stimulus was introduced by presenting it 500-700 ms earlier than the attended face, allowing it to control neural resources before the latter was presented. To our knowledge, the current study is the first to show competitive effects in the ERP between simultaneously presented stimuli while visual attention is directed away. Critically, the size of these effects depended 
on the spatial separation between the stimuli, suggesting that they scale with RF size within an area (Bles et al., 2006; Kastner et al., 2001 a; Pinsk et al., 1999), as is suggested by biased competition accounts of visual attention (Desimone \& Duncan, 1995; Kastner \& Ungerleider, 2001b). Note that this is effect is not a straightforward prediction for e.g. spotlight theories of attention. Previous studies have found that P1 amplitude decreases when stimuli are displayed further away from the locus of attention (Mangun \& Hillyard, $1987 ;$ 1988). Since the average distance from the attend location is smaller in $2^{\circ}$ displays than in $4^{\circ}$ or $7^{\circ}$ displays (both when attention is focused at fixation and when it is focused on a picture), one would expect larger P1 amplitudes for $2^{\circ}$ displays. Instead, displaying stimuli simultaneously, we observed decreased P1 amplitudes when spatial separation was small. We suggest that these decreased amplitudes reflect competitive interactions between stimuli in close proximity of each other.

The P1 component has often been found to be the first in which attention effects can be observed in the ERP (Clark \& Hillyard, 1996; Di Russo et al., 2003; Hopfinger \& West, 2006; Luck, 1995; Mangun \& Hillyard, 1991; Martinez et al., 2001). Interestingly, no P1 attention effects were observed in the present study, nor did attention interact with spatial separation. The absence of any attentional effect at this component indicates that, in order for the biasing signal to affect competitive processes, a rudimentary analysis of the scene is first evaluated by higher-order cortical areas, which then send a top-down biasing signal back to visual processing areas to resolve stimulus competition where necessary. Support for this hypothesis comes from the fact that the first attention effects present in the ERPs were observed at the frontal N1 component. Spatial separation effects were also observed at the anterior $\mathrm{N} 1$ component. More negative amplitudes were observed for attended than unattended displays, as well as for smaller spatial separations compared to larger ones. The effects of spatial separation and attention at this anterior N1 component could arise from attentional orienting networks at frontal and parietal sites (Di Russo et al., 2003; Kastner et al., 2001 a; Kastner \& Ungerleider, 2001 b; Martinez et al., 1999; Martinez et al., 2001). This biasing signal is stronger for attended conditions than for unattended conditions (in which competition resolution is not strictly necessary), and for small spatial separations compared to high ones.

44 The influence of the biasing signal could be observed in the $\mathrm{N} 1$ component at posterior sites, which has reached its maximum about $30 \mathrm{~ms}$ after the anterior $\mathrm{N} 1$. In line with previous findings (Martinez et al., 2001), this suggests that this component is associated with a re-entrant sweep of activity through the visual cortex, which is modulated by top-down attention mechanisms. Here too, attended conditions led to more negative amplitudes, as did smaller spatial separations. Critically, attention and spatial separation displayed a significant interaction at parietal and occipital scalp sites: the effect of attention 
was larger in displays with small spatial separations, compared to larger ones. In line with a biased competition account of visual attention, this implies that the amount of competition that is resolved by attention depends on the spatial separation between stimuli.

Finally, attention effects were observed in the subsequent P2 and late negativity components. In addition, spatial separation effects were observed in the P2 and the late part of the late negativity (310-450 ms). Although these results are difficult to interpret in light of the exact underlying mechanisms, the persistence of these effects into these late time windows, indicates that higher-order stimulus evaluation takes place until well after stimulus offset (at $250 \mathrm{~ms}$ ). The difference between attended and unattended displays in these late time windows could reflect task-specific activations associated with bar-width discrimination on the one hand, and picture recognition in the other. The effect of spatial separation in this time window indicates that the influence of stimulus competition remains detectable long after stimulus onset, possibly by requiring different resource quantities under different competitive conditions.

In summary, in the present study, a paradigm that has been employed before in monkey neurophysiology and human neuroimaging studies was adapted for use in a human ERP setting. It enabled, for the first time, the disentangling of competitive and biasing effects within human visual cortex. This study revealed that, initially, only effects of stimulus competition can be observed in the ERP. After about $130 \mathrm{~ms}$, attention effects emerge, presumably from a frontal-parietal attention network. The top-down signal sent back from this network to the visual cortex then influences stimulus processing in posterior areas, visible in an attentional modulation of the posterior N1 component which varies with spatial separation and hence with the amount of competition between stimuli.

\section{Acknowledgements}

The authors wish to thank Olga Reneerkens, Stefanie Pfeiffer and Ruben Janssen for their help in testing the participants. This project was supported by NWO ASPASIA grant nr. 015.001 .053 to B.M. Jansma (Schmitt). 


\section{References}

Beck, D. M., \& Kastner, S. (2005). Stimulus context modulates competition in human extrastriate cortex. Nat Neurosci 8, $1110-1116$.

Bentin, S., McCarthy, G., Perez, E., Puce, A., \& Allison, T. (1996). Electrophysiological studies of face perception in humans. Journal of Cognitive Neuroscience 8, 551 565.

Bles, M., Schwarzbach, J., De Weerd, P., Goebel, R., \& Jansma, B. M. (2006). Receptive field size-dependent attention effects in simultaneously presented stimulus displays. Neuroimage 30, 506-511.

Boussaoud, D., Desimone, R., \& Ungerleider, L. G. (1991). Visual topography of area TEO in the macaque. J Comp Neurol 306, 554-575.

Broadbent, D. (1958). Perception and communication (London, Pergamon Press).

Chelazzi, L., Duncan, J., Miller, E. K., \& Desimone, R. (1998). Responses of neurons in inferior temporal cortex during memory-guided visual search. Journal of Neurophysiology 80, 2918-2940.

Chelazzi, L., Miller, E. K., Duncan, J., \& Desimone, R. (2001). Responses of Neurons in Macaque Area V4 During Memory-guided visual Search. Cerebral Cortex 11, 761-772.

Clark, V. P., \& Hillyard, S. A. (1996). Spatial selective attention affects early extrastriate but not striate components of the visual evoked potential. Journal of Cognitive Neuroscience 8, 387-402.

Desimone, R., \& Duncan, J. (1995). Neural mechanisms of selective visual attention. Annu Rev Neurosci 18, 193-222.

Di Russo, F., Martinez, A., \& Hillyard, S. A. (2003). Source analysis of event-related cortical activity during visuo-spatial attention. Cerebral Cortex 13, 486-499.

Gattass, R., Gross, C. G., \& Sandell, J. H. (1981). Visual topography of V2 in the macaque. J Comp Neurol 201, 519-539.

46 Gattass, R., Sousa, A. P., \& Gross, C. G. (1988). Visuotopic organization and extent of V3 and V4 of the macaque. J Neurosci 8, 1831-1845.

Greenhouse, S., \& Geisser, S. (1959). On methods in the analysis of profile data. Psychometrika 24, 95-112.

Henson, R. N., Goshen-Gottstein, Y., Ganel, T., Otten, L. J., Quayle, A., \& Rugg, M. D. (2003). Electrophysiological and haemodynamic correlates of face perception, recognition and priming. Cereb Cortex 13, 793-805.

Hillyard, S. A., \& Anllo-Vento, L. (1998). Event-related brain potentials in the study of visual selective attention. Proc Natl Acad Sci U S A 95, 781-787. 
Hillyard, S. A., Vogel, E. K., \& Luck, S. J. (1998). Sensory gain control (amplification) as a mechanism of selective attention: electrophysiological and neuroimaging evidence. Philos Trans R Soc Lond B Biol Sci 353, 1257-1270.

Hopf, J. M., Boelmans, K., Schoenfeld, A. M., Heinze, H. J., \& Luck, S. J. (2002). How does attention attenuate target-distractor interference in vision?. Evidence from magnetoencephalographic recordings. Brain Res Cogn Brain Res 15, 17-29.

Hopf, J. M., Schoenfeld, M. A., \& Heinze, H. J. (2005). The temporal flexibility of attentional selection in the visual cortex. Curr Opin Neurobiol 15, 183-187.

Hopfinger, J. B., \& West, V. M. (2006). Interactions between endogenous and exogenous attention on cortical visual processing. Neuroimage 31, 774-789.

Jacques, C., \& Rossion, B. (2004). Concurrent processing reveals competition between visual representations of faces. Neuroreport 15, 2417-2421.

Jacques, C., \& Rossion, B. (2006a). Electrophysiological Evidence for Temporal Dissociation between Spatial Attention and Sensory Competition during Human Face Processing. Cereb Cortex.

Jacques, C., \& Rossion, B. (2006b). The time course of visual competition to the presentation of centrally fixated faces. J Vis 6, 154-162.

Kastner, S., De Weerd, P., Desimone, R., \& Ungerleider, L. (1998). Mechanisms of Directed Attention in the Human Extrastriate Cortex as Revealed by Functional MRI. Science 282, 108-111.

Kastner, S., Pinsk, M. A., De Weerd, P., Desimone, R., \& Ungerleider, L. (1999). Increased Activity in Human Visual Cortex during Directed Attention in the Absence of Visual Stimulation. Neuron 22, 751-761.

Kastner, S., De Weerd, P., Pinsk, M. A., Elizondo, M. I., Desimone, R., \& Ungerleider, L. (2001a). Modulation of Sensory Suppression: Implications for Receptive Field Sizes in the Human Visual Cortex. Journal of Neurophysiology 86, 1398-1411.

Kastner, S., \& Ungerleider, L. (2001 b). The neural basis of biased competition in human visual cortex. Neuropsychologia 39, 1263-1276.

Luck, S. J., \& Hillyard, S. A. (1994). Electrophysiological correlates of feature analysis during visual search. Psychophysiology 31, 291-308.

Luck, S. J. (1995). Multiple mechanisms of visual-spatial attention: recent evidence from human electrophysiology. Behav Brain Res 71, 113-123.

Luck, S. J., Chelazzi, L., Hillyard, S. A., \& Desimone, R. (1997). Neural mechanisms of spatial selective attention in areas V1, V2, and V4 of macaque visual cortex. J Neurophysiol 77, 24-42.

Mangun, G. R., \& Hillyard, S. A. (1987). The spatial allocation of visual attention as indexed by event-related brain potentials. Hum Factors 29, 195-211. 
Mangun, G. R., \& Hillyard, S. A. (1988). Spatial gradients of visual attention: behavioral and electrophysiological evidence. Electroencephalogr Clin Neurophysiol 70, 417-428.

Mangun, G. R., \& Hillyard, S. A. (1991). Modulations of sensory-evoked brain potentials indicate changes in perceptual processing during visual-spatial priming. J Exp Psychol Hum Percept Perform 17, 1057-1074.

Martinez, A., Anllo-Vento, L., Sereno, M. I., Frank.L.R., Buxton, R. B., Dubowitz, D. J., Wong, E. C., Heinze, H. J., \& Hillyard, S. A. (1999). Involvement of striate and extrastriate visual cortical areas in spatial attention. Nature Neuroscience 2,364369.

Martinez, A., DiRusso, F., Anllo-Vento, L., Sereno, M. I., Buxton, R. B., \& Hillyard, S. A. (2001). Putting spatial attention on the map: timing and localization of stimulus selection processes in striate and extrastriate visual areas. Vision Res 41, 1437 1457.

Miller, E. K., Gochin, P. M., \& Gross, C. G. (1993). Suppression of visual responses of neurons in inferior temporal cortex of the awake macaque by addition of a second stimulus. Brain Res 616, 25-29.

Moran, J., \& Desimone, R. (1985). Selective attention gates visual processing in the extrastriate cortex. Science 229, 782-784.

Noesselt, T., Hillyard, S. A., Woldorff, M. G., Schoenfeld, A., Hagner, T., Jancke, L., Tempelmann, C., Hinrichs, H., \& Heinze, H. J. (2002). Delayed striate cortical activation during spatial attention. Neuron 35, 575-587.

Nuwer, M. R. Comi, C., Emerson, R., Fuglsang-Frederiksen, A., Guerit, J. M., Hinrichs, H., Ikeda, A., Lucca, F. J., Rappelsburger, P. (1998). IFCN standards for digital recording of clinical EEG. Electroencephalogr Clin Neurophysiol 106, 259-61.

Pinsk, M. A., Kastner, S., Desimone, R., \& Ungerleider, L. (1999). An estimate of receptive field sizes in human visual cortex. Neurolmage 9, s885.

Recanzone, G. H., Wurtz, R. H., \& Schwarz, U. (1997). Responses of MT and MST neurons to one and two moving objects in the receptive field. J Neurophysiol 78, 29042915.

Reynolds, J. H., Chelazzi, L., \& Desimone, R. (1999). Competitive mechanisms subserve attention in macaque areas V2 and V4. J Neurosci 19, 1736-1753.

Smith, A. T., Singh, K. D., Williams, A. L., \& Greenlee, M. W. (2001). Estimating receptive field size from fMRI data in human striate and extrastriate visual cortex. Cereb Cortex 11, 1182-1190.

Treisman, A. M. (1969). Strategies and models of selective attention. Psychol Rev 76, 282-299. 
Woodman, G. F., \& Luck, S. J. (1999). Electrophysiological measurement of rapid shifts of attention during visual search. Nature 400, 867-869. 
O
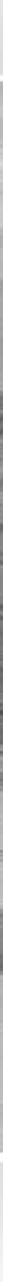


\section{Phonological processing of ignored distractor pictures, an fMRI investigation}

Neuroimaging studies of attention often focus on interactions between stimulus representations and top-down selection mechanisms in visual cortex. Less is known about the neural representation of distractor stimuli beyond visual areas, and the interactions between stimuli in linguistic processing areas. In this study, participants viewed simultaneously presented line drawings at peripheral locations. The names of the objects depicted in these pictures were either phonologically related (i.e. share the same consonant-vowel onset construction), or unrelated. Attention was directed either at the linguistic properties of one of these pictures, or at the fixation point (i.e. away from the pictures). Results showed that phonological representations of unattended pictures could be detected in the posterior superior temporal gyrus, the inferior frontal gyrus, and the insula. Hence, selective attention to a specific location does not completely filter out the representations of distractor stimuli, but rather attenuates them. 


\section{Introduction}

A typical property of human perception is its selective nature. Only a limited amount of information from our environment reaches our awareness, indicating that our brain selects some objects for further processing, while ignoring others. It is generally assumed that the purpose of this selection is the more efficient processing of selected stimuli than would be the case if all stimuli were processed simultaneously. Most attention research has therefore focused on the enhanced processing of attended stimuli, showing that attended stimuli are easier to detect and identify. Considerably less is known, however, about the fate of ignored stimuli. The main target of this study was to investigate whether information about ignored distractor stimuli becomes available in brain areas beyond typical object processing areas. Specifically, we investigated whether phonological areas activate the phonological code of ignored distractors. Accordingly, we gain insight in selection mechanisms both at a visual and a phonological level.

It is generally assumed that the necessity for selection depends on the limited processing capacity of our sensory systems, which prevents each stimulus to be represented to the full extent. According to the influential 'biased competition' approach, simultaneously presented stimuli compete for neural representation and behavioural control, and engage in suppressive interactions (for an overview, see Chelazzi, 1999; Desimone, 1998; Duncan, 2006; Kastner \& Ungerleider, 2001 b). For example, Kastner et al. (1998; 1999; 2001 a; Pinsk et al., 1999) have shown that when participants viewed simultaneously presented stimuli, BOLD-signal change was reduced compared to sequential viewing of the same stimuli (where no suppressive interactions could take place). These results support the view that simultaneously presented stimuli suppress each other's neural representation, and thereby reduce the magnitude of the BOLD response. However, competition can be biased in favour of a stimulus through bottom-up or top-down mechanisms. In such a case, this stimulus will be more successful in suppressing the representation of the others, and its neural representation will take precedence over that of the others. Indeed, neural suppression effects diminished when one of the stimuli was very salient (Beck \& Kastner, 2005) or when one of the stimuli was attended (Bles et al., 2006; Kastner et al., 1998; Kastner et al., 1999; Pinsk et al., 1999), showing that these stimuli had succeeded in overcoming the suppressive effects of the distractor stimuli.

An issue that has occupied researchers for decades is what happens to the neural representation of the distractor stimuli. Is the representation of these stimuli abolished, or do (attenuated) representations exist beyond the areas in which competition takes place? In the former case, attention is believed to select certain stimuli from the environment at the perceptual level, while ignoring irrelevant distractor stimuli. Since these distractor stimuli are filtered out at a perceptual level, the brain is unaware of their identity, and participants are typically unable to report many characteristics about these stimuli. An example of early 
selection is given by the dichotic shadowing paradigm (e.g. Broadbent, 1958; Cherry, 1953), in which participants are presented with a separate auditory stream in each ear and are told to repeat the words heard in one channel, and ignore the words presented to the other. Typically, participants are unable to report any of the words presented in the ignored channel. Most participants are even unaware if the language in the unattended ear suddenly changes, although a change of gender of the speaker is generally noted, as is the appearance of a sine-wave tone (Cherry, 1953). Further evidence for early selection theories comes from visual search paradigms, where participants are generally able to locate targets on the basis of physical properties such as location or size, but not semantic properties like alphanumerical category (Sperling, 1960). This indicates that, although some physical characteristics of (unattended) perceptual channels are extracted automatically, none of the semantic properties of the unattended channels are activated.

Proponents of late selection theories, instead, argue that all stimuli are perceptually processed and identified, but that selection takes place at a higher-order level like working memory representation or response selection. In this case, attention serves as a filter for determining which perceived objects reach awareness. Traditionally, evidence for late selection theories comes from investigations that show that some features of ignored stimuli influence overt behaviour. In order for ignored distractors to be able to interfere with overt responses, knowledge about their identity must be known by the brain, indicating that these stimuli have received at least some processing resources. A classical example of late selection is the famous 'cocktail party effect'. As reported earlier, people taking part in shadowing tasks generally cannot report any of the semantic characteristics of an unattended auditory stream. However, about one third of the participants does notice when his or her name is presented to the unattended channel, indicating that some semantic processing of this stream must take place (Moray, 1959; Wood \& Cowan, 1995). Similarly, when a judgment needs to be made about the identity of a target letter at fixation, responses are generally faster when ignored flanker items are congruent with the target letter than when they are incongruent, indicating that the flankers are identified (e.g. Bradshaw, 1974; Eriksen \& Eriksen, 1974).

Recently, some authors have tried to resolve discrepancies between these views and integrate early and late selection mechanisms (e.g. Lavie, 1995; Lavie et al., 2004; Vogel et al., 2005). These frameworks postulate that attention can filter distractor information either at an early or a late level, depending on the available resources and task requirements. Typically, attention acts at the perceptual level in cases where the perceptual system is overloaded and a selection needs to be made which information is further processed. When such selections are not necessary, however, irrelevant stimuli will also be processed by sensory areas, but attention prevents them from being represented in working memory, thereby blocking awareness of these stimuli. According to these theories, identification of irrelevant stimuli occurs in cases in which resource demands are low. 
Neuroimaging techniques provide a good method of choice to trace the processing of ignored distractors within the brain, since they enable the mapping of influences of stimuli throughout several cortical areas (with fMRI) without the need for a participant's overt response. Some investigators have indeed detected activation traces associated with ignored distractors beyond early visual areas. For example, some studies have shown that the decreased BOLD response typically associated with stimulus repetition also occurs with repetition of unattended stimuli (albeit smaller) (Bentley et al., 2003; Murray \& Woiciulik, 2004; Yi et al., 2004). In line with load theory (Lavie et al., 2004), these effects disappeared when task demands were high (Eger et al., 2004; Yi et al., 2004; Yi et al., 2006), suggesting that ignored stimuli are involuntarily processed in case task demands do not require all available resources. Ruz et al. (2005) observed that ERPs differentiated between words and non-words even though attention was highly engaged by another task (but see Rees et al., 1999). According to the authors, these results indicate that, at least for highly learned stimuli such as letter strings, some processing resources are automatically dedicated even though participants are engaged in an attentionally demanding task.

The goal of the present study is to investigate whether cortical areas beyond visual areas engage in the processing of ignored stimuli. Specifically, we investigated whether phonological relatedness between names of different pictures influences processing in regions that process meaning and phonology, and whether this effect was dependent on the location of attention. To this end, a design that has proven successful in visual attention research (Bles et al., 2006) was modified. Sets of pictures were presented simultaneously at peripheral locations of the visual field, while participants performed one of three linguistic tasks in which they attended the picture, or a central task at fixation (i.e. ignore the picture). Of the three attended tasks, two focussed on phonological processing (onset monitoring, offset monitoring), and one focused on meaning (semantic category judgement). The fixation task aimed to withdraw attention from the pictures and involved a visual line discrimination. Whereas this design was previously employed to measure suppression effects between abstract, colourful stimuli in visual cortex, the present adaptation entails the use of concrete black-and-white line drawings. By assigning meaning to pictures, it becomes possible to measure hemodynamic responses

54 to the pictures in cortical areas associated with semantic and phonological processing. Moreover, we manipulated the amount of phonological overlap between the names of the simultaneously presented pictures. In the 'related' condition, the names of the pictures consisted of at least an identical first consonant-vowel construction, e.g. a picture of a "VOS" (fox) and a picture of a "VORK" (fork), see figure 4.1). In the 'unrelated' condition, there was no such phonological overlap (a picture of a "VOS" (fox) and a picture of a "HARK" (rake)). The underlying rationale is that if phonological processing areas respond differently to related sets than to unrelated sets, the phonological properties of both target and distractor pictures must have been identified. This would imply that, at least under 


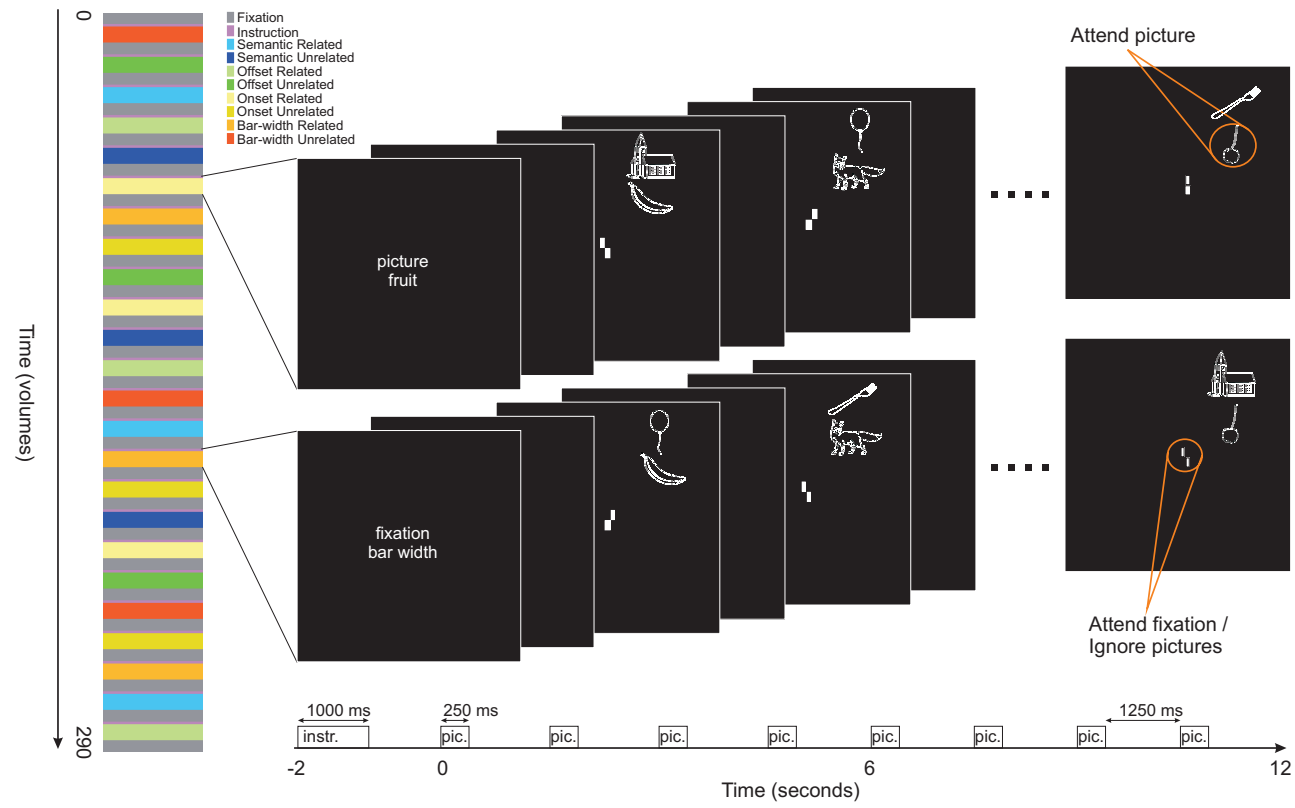

Figure 4.1 Experimental design. Each participant performed four functional runs, including 24 task blocks each. These included three linguistic tasks (onset monitoring, offset monitoring, semantic categorisation) in which the lower picture was attended. In the perceptual bar-width discrimination task, the pictures were ignored. Blocks were either phonologically related (e.g. kers - kerk ('cherry' - 'church') or unrelated (e.g. kers - vork ('cherry' - 'fork')), leading to a 4 (task) * 2 (relatedness) design, which was repeated 3 times per run in a semirandomised order.

these circumstances, even though visual attention is highly focused on a single object or location, attention attenuates the processing of unattended objects, rather than filter them out completely.

We expected to observe effects of phonological relatedness mainly in cortical areas involved with generating the pictures' names. Specifically, we were interested in the left posterior superior temporal gyrus (STG) and the left anterior insula, which are presumably involved in phonological code retrieval (Indefrey \& Levelt, 2004; Noesselt et al., 2003), and in the left inferior frontal gyrus (IFG), believed to be involved in syllabification and phonological processing (Bookheimer, 2002; Fiez, 1997; Hagoort, 2005; Indefrey \& Levelt, 2004). The left posterior STG (often referred to as Wernicke's area), has been observed to show decreased activation in a picture-word interference paradigm when phonologically pictures were presented with related distractor words, compared to unrelated words (de Zubicaray et al., 2002). Assuming that the lower BOLD response is linked to a reduced amount of neural processing, this observation is in line with the observation that response times are generally faster in phonologically related trials (Jescheniak \& Schriefers, 2001; Peterson \& Savoy, 1998; Starreveld \& La Heii, 1996).

In addition to the question whether relatedness effects would be observed in phonological processing areas, we were interested in the interaction of this effect with the task a participant is performing. In particular, whereas it is quite straightforward to 
anticipate relatedness effects in the two phonological tasks, the question was whether phonological relatedness also affects processing during non-phonological semantic categorisation, or a visual line comparison task. In the onset monitoring task, participants were asked to judge whether the name of the picture closest to the fixation point started with a certain letter. In order to carry out this task, participants had to internally generate at least the first part of the name of the picture. This task most directly aimed to test effects of distractor relatedness, as the experimental relatedness between target and distractor was realised at this onset-letter position. The offset monitoring task was similar, but involved the decision whether the picture name ended with a certain letter. In order to perform this task, participants had to generate the entire name of the target, including the onset of the name, in which overlap exists with the onset of the distractor picture's name in related trials. The semantic task involved a category judgment in which participants responded to pictures belonging to a certain category (e.g. mammals, musical instruments). Participants were not required to generate the pictures' names for successful performance in this task. The control task (ignore the picture) involved an attentionally demanding line comparison task at fixation. This condition served as a visual control, with exactly the same physical stimulation parameters as the other tasks, but without participants attending one of the pictures.

In this design, areas that are involved in phonological encoding, such as the STG and the posterior IFG, were expected to be activated stronger in the phonological tasks than in the semantic or control task. The question was whether within those areas effects of phonological relatedness could be observed, indicating that the names of ignored pictures are activated. Furthermore, we investigated whether those effects were also visible in the same areas in non-phonological (i.e. semantic category judgment and line comparison) tasks, indicating that name generation occurs even when phonological processing is not required. Finally, we were interested whether phonological relatedness effects would be observed in areas involved in semantic processing, such as the left prefrontal cortex and the left middle temporal gyrus (see Cabeza \& Nyberg (2000) for a review), and visual processing, (see Goebel et al. (2003)). This requires exact localisation of early visual areas and the Lateral Occipital Complex, involved in object recognition (Grill-Spector et 56 al., 1998; Grill-Spector et al., 2001). Therefore, standard retinotopy and LOC localiser runs were included in the experiment'. Finding effects of phonological relatedness in these areas would imply that, presumably through top-down biasing signals, phonological features can influence picture processing in non-phonological areas.

\footnotetext{
1 An fMRI pilot study involving twelve participants and only the onset and the control task indicated that, in the onset monitoring task, effects of relatedness could be observed in areas along the calcarine sulcus and fusiform gyrus. Since no localisers for visual areas were included in this experiment, effects were hard to locate precisely in a functional manner. The offset monitoring and categorisation task were added to test under which task conditions relatedness effects were observed. Finally, since effects of relatedness were unexpectedly found in visual areas, the number of pictures in a display was reduced from four to two, in order to be able to better control physical stimulation parameters.
} 


\section{Methods}

\section{Partic ipants}

Fourteen volunteers (6 Females, age 20-31 years) participated in this study. All were right-handed, neurologically healthy and had normal or corrected-to-normal visual acuity and were paid for their time. This research project was approved by the local ethics committee.

\section{Stimuli}

Twenty-four pairs of white line drawings of common objects on a black background were used as stimuli in this experiment. The names of the pictures within a pair were phonologically related, i.e. they shared at least the same consonant-vowel onset structure with each other, e.g. "VOS" (fox), "VORK" (fork) (average word length 5 letters, average onset overlap 2.2 letters, see appendix 4.1). The stimuli were divided into three sets of eight pairs, encompassing the stimuli for the 'related' blocks. The same sets were recombined into 'unrelated' sets, so that the members were phonologically and semantically unrelated and did not have offset overlap. One member of each pair was designated as an attended picture (i.e. occurring at the location closest to the fixation point), and one as a distractor picture (occurring at the location furthest away from the fixation point). Within each set of eight attended pictures, two targets were designated per task (i.e. required an overt response). E.g. a set of eight attended pictures might have two pictures starting with a ' $r$ ' (onset task), two ending with a ' $\mathrm{g}$ ' (offset task), and two buildings (categorisation task). Target trials in the fourth task (bar-width discrimination) had the same picture pairs in the display as the three other tasks but were defined based on the properties of the bars at the centre of the screen. Each set of 'attended' pictures was presented eight times: once for each of the four tasks both in phonologically related and in unrelated conditions. Hence, visual stimulation and amount of targets per block across these tasks was identical; the only parameter that differed was task instruction.

In related blocks, an 'attended' picture was presented with the distractor picture that was phonologically related to it. Unrelated blocks were created by pairing 'attended' pictures of one set with the distractor pictures of another set. This insured that these new combinations were phonologically unrelated. Care was taken to avoid any semantic relationship or offset overlap within unrelated pairs. Each picture that appeared at a distractor position in a related block, appeared at the same position, but paired with a different 'attended' picture in an unrelated block. The same holds for 'attended' pictures. Each of these sets was presented 4 times per fMRI run: once per task (onset monitoring, 
offset monitoring, semantic judgement, bar-width discrimination). This way, it was assured that across tasks visual stimulation was identical. In addition, the number of targets across blocks was kept constant and targets never occurred at distractor locations.

Distractor pictures never matched target criteria for a certain condition, were always semantically unrelated to the 'attended' picture, and had a different name offset. In related conditions, they shared the initial consonant vowel structure with the target. In unrelated conditions, they did not share onset phonemes with the target.

\section{Procedure}

Trials were presented in a block-design. An fMRI run started with the presentation of a blank screen for 18 seconds, followed by an instruction centred on the screen for 1 second, consisting of two keywords indicating the target location and the target. Subsequently, another blank screen followed for 1 second, after which a block started. Blocks consisted of the presentation of eight trials of two stimuli simultaneously presented above each other in the upper right quadrant of the screen for $250 \mathrm{~ms}$, followed by a blank screen for 1250 ms, after which a new pair of stimuli was presented. The 'attended' picture was centred at $2.5^{\circ}$ from the fixation cross, the distractor stimulus $2^{\circ}$ degrees above it. Each picture subtended approximately $1.5^{\circ}$ degrees of visual angle.

During stimulus presentation, participants performed one of four tasks, indicated to them prior to the start of the block. In the onset monitoring task, participants were asked to judge whether the name of the picture closest to the fixation point started with a certain letter. The offset monitoring task was similar, but involved the decision whether the picture name ended with a certain letter. The semantic task involved a category judgment in which participants responded to pictures belonging to a certain category (e.g. mammals, musical instruments). The control task involved a bar-width discrimination task: participants were asked to attend the two bars (. $2^{\circ}$ in height) that replaced the fixation cross. These bars were presented $.15^{\circ}$ degrees above and below the fixation cross. The width of the bars varied between 1 and 5 pixels, and the difference between the bars was never more than 1 pixel. A button press was required in case the bars were of the same width (2 targets per block).

58 Participants were instructed to fixate the centre of the screen (fixation cross) throughout the experiment and to minimise head and eye-movements while in the scanner. A previous ERP pilot experiment involving a similar design monitored eye movements, and revealed that participants were able to maintain fixation across conditions. They were not made aware of the phonological relationship between the stimuli and were asked to ignore the distractor stimuli.

The order of the blocks was randomised for each run and each participant. Random permutations of the eight conditions were repeated three times per run, with the restriction that the same task never appeared twice in succession. 


\section{Scanning parameters}

Images were acquired on a 3T Siemens Allegra head scanner (Siemens Medical Systems, Erlangen, Germany) using a standard head coil. Thirty-two oblique axial slices (in-plane resolution: $3.5 \times 3.5 \mathrm{~mm}$, slice thickness: $3.5 \mathrm{~mm}$, interslice distance $0 \mathrm{~mm}$ ) covering the entire cortical volume were acquired using an echo planar imaging sequence (TR $=2000 \mathrm{~ms}, \mathrm{TE}=29 \mathrm{~ms}$, matrix size: $64 \times 64$ ). There were 268 volumes per run, the first 2 of which were skipped due to the T1 saturation effect. Functional slices of each run were aligned to a high resolution (voxel size $1 \times 1 \times 1 \mathrm{~mm}^{3}$ ) anatomical dataset acquired using a $\mathrm{Tl}$-weighted 3D MP-RAGE (magnetization-prepared rapid acquisition gradient echo) sequence (192 sagittal slices, $T R=2.3 \mathrm{~s}, \mathrm{TE}=3.93 \mathrm{~ms}$ ).

The participants were placed comfortably in the scanner and their head was fixated with foam pads. Mounted on the head coil was a mirror through which they could see the stimuli projected on a screen. Stimulus presentation was synchronised with MR data acquisition by triggering the stimulus program with the first MR pulse.

\section{Region of interest localisation}

Polar maps were acquired using a rotating, red-green blocked wedge of 33.75 degree polar angle covering eccentricities from $1^{\circ}$ to $17^{\circ}$ of visual angle. Wedges were filled with a checkerboard pattern of red and green squares that reversed polarity 8 times per second and did a full rotation within 64 seconds. Thus each pixel in a circular field of view was activated every 64 seconds for a duration of 6 seconds. A functional run took 552 seconds, i.e. 8 cycles of rotating wedges plus 20 seconds lead in and lead out time. Borders between areas were defined based on the alternating vertical and horizontal meridians that demarcate the borders of these areas (Goebel et al., 2003; Sereno et al., 1995). Ventral visual areas V1, V2, VP and V4 were defined for each participant. Area TEO could not be reliably located for most participants by this method, but was defined as the area anterior to V4 that was activated reliably by the stimuli across participants, and was located at comparable Talairach coordinates as in previous studies (Bles et al., 2006; Kastner et al., 1998).

The LOC was localised by contrasting blocks of objects with blocks of scrambled objects (FDR-corrected, $q=.05$ ). Each participant viewed 2 runs of this localiser which contained 3 blocks of objects and 3 blocks of scrambled objects, lasting $20 \mathrm{~s}$ each, separated by $15 \mathrm{~s}$ baseline intervals. Scrambled objects were created by dividing each object picture in a 20 by 20 square grid and randomly rearranging the grid elements.

Scanning parameters were the same for the localiser runs and the experimental runs. Preprocessing procedures were also identical, except that no spatial smoothing was applied. 


\section{Analysis}

Data were analysed using the BrainVoyager QX package (Brain Innovation, Maastricht, The Netherlands). Functional data were corrected for motion in three dimensions, slice scan-time corrected and spatially smoothed with a $4 \mathrm{~mm}$ FWHM Gaussian kernel. Subsequently, linear drifts were removed from the signal and data were high-pass filtered to remove slow frequency drifts up to 3 cycles per time course. After preprocessing, functional data were aligned to the high-resolution anatomical images, morphed to fit Talairach dimensions (Talairach \& Tournoux, 1988) and combined into a 4-dimensional dataset for each run and each participant.

In order to improve anatomical correspondence between participants, all individual brains were segmented at the gray/white matter boundary (using a semi-automatic procedure based on intensity values) and the reconstructed cortices were aligned using curvature information reflecting the gyral/sulcal folding pattern. The reconstructed folded cortical representations of each participant and hemisphere were morphed into a spherical representation, each vertex on the sphere (spherical coordinate system) corresponding to a vertex of the folded cortex (Cartesian coordinate system) and vice versa. The curvature information computed in the folded representation was preserved as a curvature map on the spherical representation, driving intercortex alignment and minimizing the mean squared differences between the curvature of a source sphere and the average of all spheres. Visual inspection and a measure of the averaged mean squared curvature difference revealed that the alignment of major gyri and sulci was achieved reliably by this method.

The established correspondence mapping between vertices of the cortices was used to align the time courses for multi-subject General Linear Model data analysis. The GLM included eight predictors: onset related, onset unrelated, offset related, offset unrelated, category related, category unrelated, control related, and control unrelated. Fixed effects (FFX) statistical maps were FDR-corrected (Genovese et al., 2002) at an FDR level of .05. Second level analysis used the average of the estimated beta weight values of each participant and condition from the FFX analysis, which were then included in a random effects (RFX) ANCOVA model with factors task (4 levels) and relatedness (2 levels). Reported RFX ANCOVA maps were uncorrected for multiple comparison. For each participant, functionally defined regions of interest (ROI) were specified based on the localiser runs. The activation in these areas was analysed with an RFX ROI approach, using functional data from each individually defined ROI. 


\section{Results}

\section{Behavioural data}

A repeated measures GLM with the factors task and relatedness revealed a significant main effect of task on reaction time $(F(3,39)=41,71, p<.001)$. Post-hoc analysis revealed that reaction times were fastest in response to the semantic task (615 ms), followed by the control task $(651 \mathrm{~ms})$, the onset $(717 \mathrm{~ms})$ and offset monitoring task (773 $\mathrm{ms}$ ), respectively. The differences between each of the tasks was significant (all p-values $<.04$, Bonferroni corrected). No effect of relatedness was observed $(F(1,13)=.05, p$ $=.82)$, nor was there any interaction between relatedness and task $(F(3,39)=.47, p=$ $.67)^{2}$. Error rates were generally low $(<2 \%)$ and did not differ across conditions.

\section{Fixed effects a na lysis}

Compared to baseline periods, the tasks reliably activated an extensive bilateral network of areas including parietal areas, occipital areas including early visual areas, inferior temporal areas including large parts of the fusiform gyrus, the posterior parts of the STS and the superior temporal gyrus (STG), the insulae, bilateral IFG and middle frontal gyri (MFG), and the medial frontal gyri. Within these areas, an extensive lateralisation of tasks was observed (see figure 4.2). The linguistic tasks (onset and offset monitoring, and the categorisation task) mainly activated left IFG and MFG, the posterior parts of the STS/STG, parietal and inferior temporal areas, and early visual areas, compared to the control task. This control task, on the other hand, mainly activated right IFG and MFG, parietal and inferior temporal areas and the right insula. Phonological tasks activated the left inferior and superior parietal lobule, left insula, left MFG and IFG (BA9 / BA44 / BA45) and the medial frontal gyrus more strongly than the semantic task. The reverse was true for the anterior IFG (BA47) and large portions of the middle temporal gyrus.

The (Unrelated > Related) contrast revealed some scattered areas throughout bilateral inferior temporal areas, the posterior parts of the STS/STG and parietal areas. However, inspection of the time courses within these areas revealed that most of these areas

\footnotetext{
2 In three pilot behavioural studies, participants were asked to respond as fast and as accurate as possible to picture names starting with a certain onset letter, to a simple symbol discrimination task (circle vs. hyphen, easy control task), or to bars of equal width (difficult control task). No effects of relatedness were observed in these studies, neither in the onset monitoring task, nor in the control tasks. One of these studies included a dual task condition in which participants were asked to respond to targets both at the target picture location and at the fixation spot. The observation that performing a dual task with the bar discrimination task led to a much stronger increase in reaction times than the symbol discrimination task indicated that the bar width discrimination task was considerably more attentionally demanding and this task was therefore used in subsequent versions of this paradigm.
} 
were only slightly activated during these tasks and that differences between relatedness conditions seemed mainly caused by baseline differences between conditions. This was confirmed by a conjunction contrast (Unrelated $>$ Related) $\cap$ (Task $>$ Baseline) which revealed a main effect of relatedness in right inferior temporal areas and a posterior area of the right MTG.
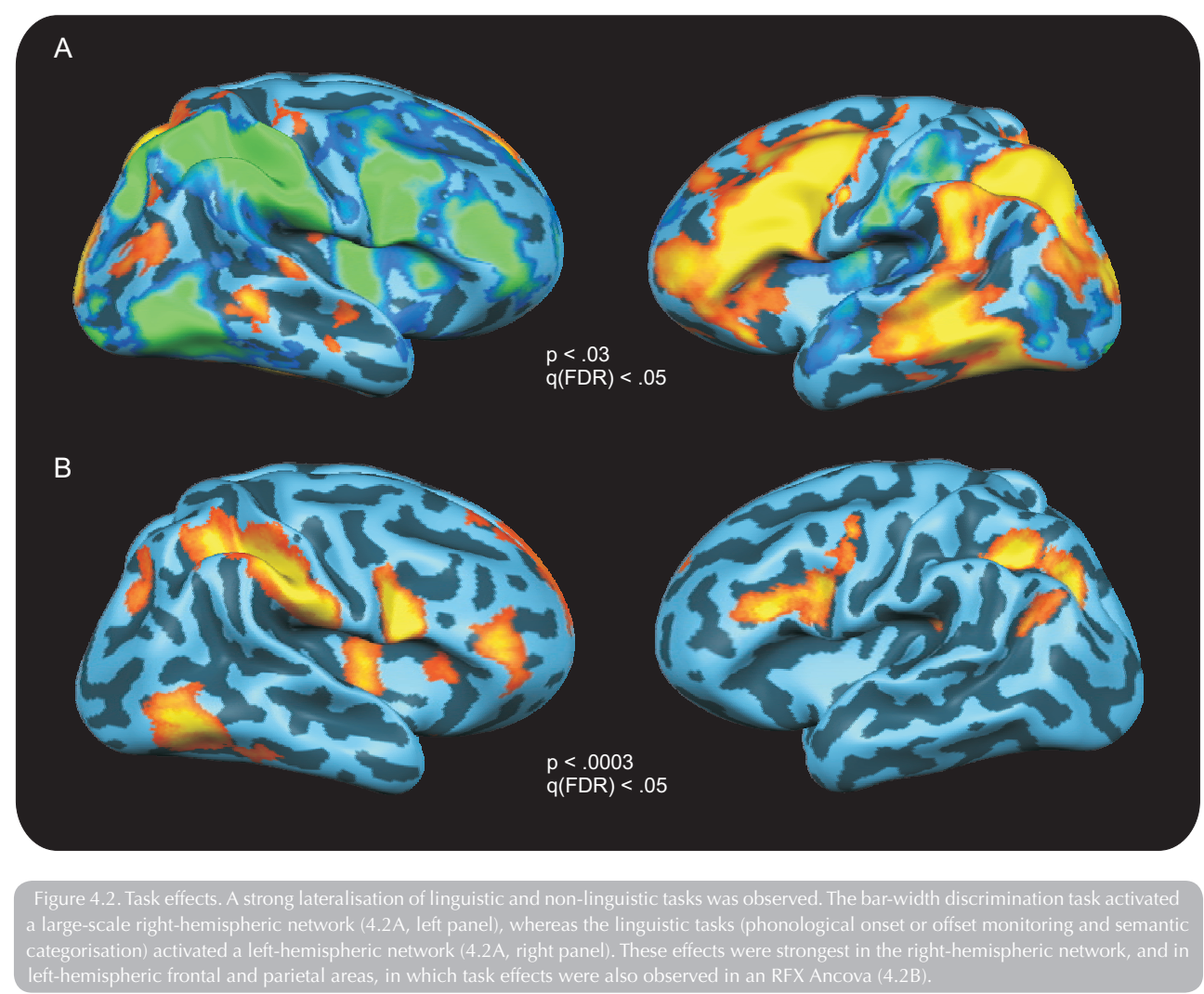

62 Due to the expected interaction between relatedness and task, we investigated relatedness effects in each task separately. In the onset task, the (Unrelated Onset $>$ Related Onset) $\cap$ (Onset $>$ Baseline) contrast revealed only the middle part of the right central sulcus.

For the offset task, the contrast (Unrelated Offset $>$ Related Offset) $\cap$ (Offset $>$ Baseline) revealed the left IFG (BA44) and an inferior part of the MFG (BA9), the posterior part of the STS/STG, bilateral precunei and bilateral inferior temporal cortices, the right cingulated gyrus and the inferior part of the left post-central gyrus (see figure 4.3a). 
No effects of relatedness were observed in the categorisation task. Both the (Unrelated Categorisation $>$ Related Categorisation) $\cap$ (Categorisation $>$ Baseline) contrast, and the reverse (Related Categorisation $>$ Unrelated Categorisation) $\cap$ (Categorisation $>$ Baseline) contrast did not reveal any areas, nor did any areas show up in the simple contrast (Unrelated Categorisation $>$ Related Categorisation) or the reverse (Related Categorisation > Unrelated Categorisation).

For the control task, the (Unrelated Central > Related Central) $\cap$ (Central $>$ Baseline) revealed bilateral anterior superior frontal gyri (SFG), as well as a the posterior part of the left middle occipital gyrus. The reverse contrast, (Related Central $>$ Unrelated Central) $\cap$ (Central > Baseline) activated the bilateral anterior parts of the insulae, bilateral MFG, bilateral medial frontal gyri, and some parts of bilateral inferior temporal cortex (see figure 4.3c).

\section{Random effects a nalysis}

The random effects ANCOVA with factors task (4) and relatedness (2) revealed a significant effect of task in bilateral inferior parietal lobules, the bilateral inferior temporal gyri, the left posterior MTG, the bilateral insulae, bilateral MFG and IFG, bilateral superior frontal cortex, and the left medial frontal gyrus $(p<.0005)$. Post-hoc analyses showed that for the left-hemispheric areas, the linguistic tasks led to a higher activation than the control task, and for right-hemispheric areas the control task led to a higher activation than the linguistic tasks.

A main effect of relatedness was observed in the right inferior parietal lobule and at the most anterior tip of the right STG $(p<.02)$. No other areas showed a significant relatedness effect in the RFX ANCOVA. An interaction between task and relatedness was observed in the left insula, the medial part of the right STG, and the right orbitofrontal cortex $(p<.05)$.

ANCOVA contrast maps of the relatedness effects within each task revealed that for the control task the left anterior insula was more active in related than unrelated conditions $(p<.005)$. For the offset task, the medial part of the right middle and superior temporal gyrus was more active in unrelated than related conditions. No relatedness effects were observed in the onset and semantic task.

Since we observed a relatedness effect in the FFX GLM in the expected left posterior middle and STS/STG and the left IFG, but did not find these areas in the RFX ANCOVA, we decided to investigate these areas with a post-hoc RFX ROI analysis. This revealed that the relatedness effect in left IFG in the offset task was consistent across participants ( $t(13)$ $=2.20, p=.046$ ). None of the other relatedness contrasts approached significance (all 
p-values $>$.3). Similarly, in the area encompassing the posterior STS/STG, a significant relatedness effect was observed for the offset task $(t(13)=2.62, p=.021)$. None of the other relatedness contrasts approached significance (all p-values $>.3$ ).

\section{Visual a reas}

Ventral visual areas V1, V2, VP and V4 were localised for each of the participants by means of polar mapping runs. In addition, the Lateral Occipital Complex (LOC) was localised with a separate run in which objects were contrasted with scrambled objects. Particpant-specific Region Of Interest (ROI) analyses (random effects model) revealed that, compared to baseline, areas V2, VP, V4 and LOC were consistently activated by all the tasks across participants (all p-values $<.01, t(13)$ ). Area $\mathrm{V} 1$ did not exhibit a significant activation increase compared to baseline ( $p>.05$ for each task). A significant effect of task was detected in $\operatorname{LOC}(F(3,39)=3.82, p=.017)$, where the linguistic tasks led to a higher activation than the control task $(t(13)=2.45, p=.029)$. Significant task effects were not observed in any of the other visual areas (all $p$-values $>.4$ ). In addition, the RFX ROI ANCOVAs did not reveal any effects of relatedness in any of the mapped visual areas, nor were any significant interactions observed between relatedness and task (all p-values $>$.3).

Possibly, any effects of our experimental manipulations in visual areas were obscured by the procedure of looking at the area as a whole, whereas only a subset of vertices reflected stimulated areas in the visual field. We therefore repeated the analyses with the subset of vertices of an area that was activated in the linguistic tasks (compared to baseline). Again, ROls were defined separately for each participant. This analysis revealed that for each participant, part of each of the ventral visual areas was activated by the stimuli in the linguistic task. Task effects were observed in areas V2, VP, V4 and LOC (all $p$-values $<.02)$, but not in $V 1(p>.1)$. Post-hoc contrasts revealed that in the areas in which task effects were observed, the linguistic tasks led to a higher activation than the control task (all p-values $<.02, t(13)$ ). No relatedness effects were observed in any of these areas, nor were there any interactions between relatedness and task (all p-values $>$

64 .3). A similar analysis based on the subset of vertices that was activated by the control task did not reveal any significant main effects or interactions in any of the areas. 


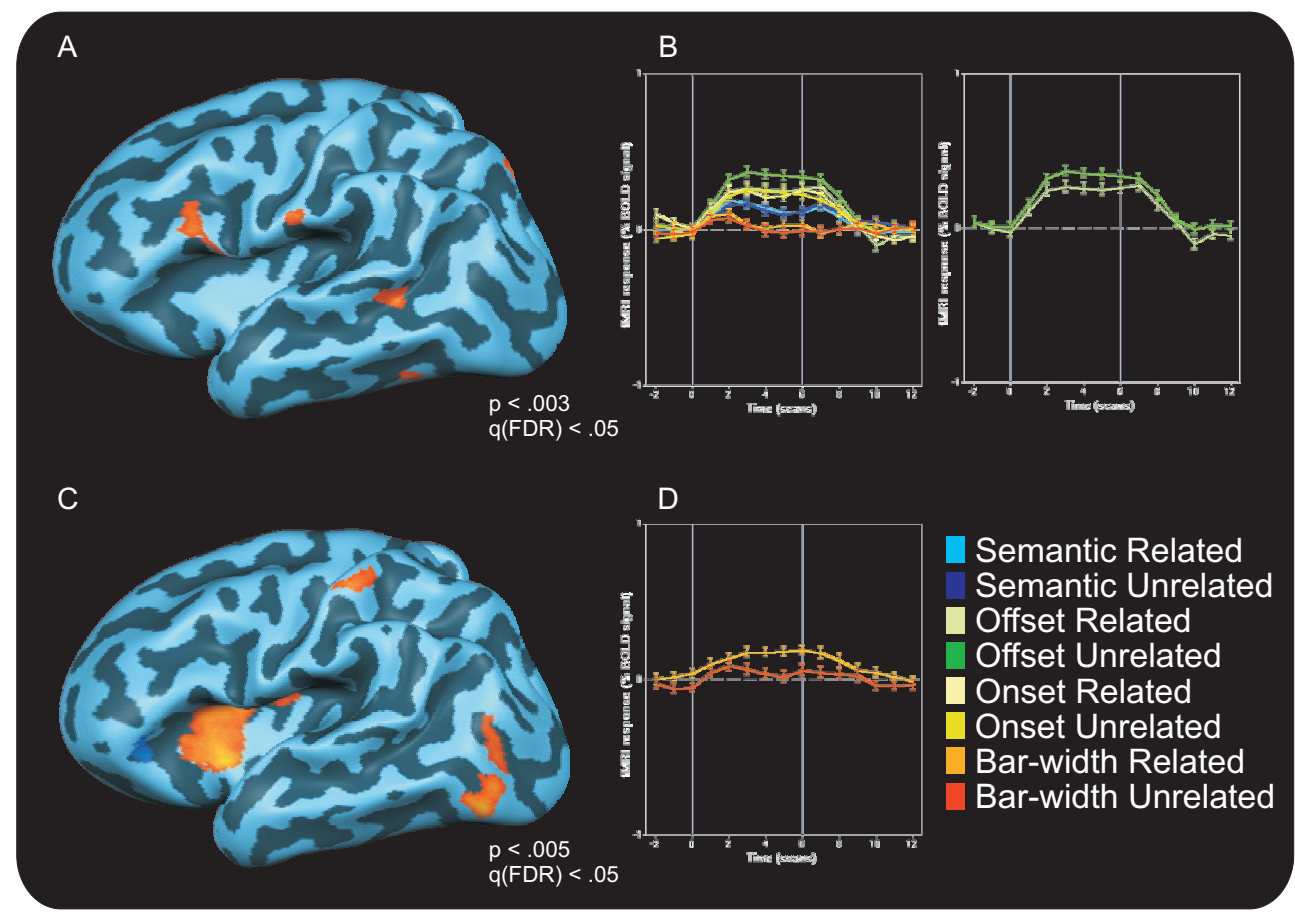

Figure 4.3. Relatedness effects. A fixed effects GLM revealed that in the offset task, related blocks led to decreased activity compared to unrelated blocks in the left IFG and STS/STG (4.3a), which was also visible in the time courses in, for example, the IFG ( $4.3 \mathrm{~b})$. In the bar-
width discrimination task, related blocks led to increased activity compared to unrelated blocks, which was most apparent in the left insula (4.3c and $4.3 d)$.

\section{Disc ussion}

The main goal of the present study was to investigate whether cortical areas beyond the visual cortex have active representations of the properties of stimuli that are to be ignored by participants. While lying in the scanner, they viewed two simultaneously presented stimuli at peripheral locations. Meanwhile, a task was performed either at fixation (ignore pictures), or concerning one of the pictures. In half of the blocks, the names of the two pictures were phonologically related to each other, in the other half they were unrelated. Whereas indications of mental representations of ignored stimuli have previously been observed in higher-order visual areas such as LOC (Murray \& Wojciulik, 2004), inferior temporal cortex (Bentley et al., 2003) and PPA (Yi et al., 2004), the present study is amongst the first to show effects of distractor processing in cortical areas involved in phonological processing, such as STS/STG, posterior IFG and the insula. 


\section{Relatedness effects}

We expected to observe relatedness effects during phonological tasks in areas that are involved in phonological processing, such as the left posterior IFG, left posterior STS/STG, and the left insula. In line with these expectations, unrelated conditions led to higher activations than related conditions in left IFG and STS/STG, when participants were performing the offset monitoring task. These effects were significant in the FFX analysis, and confirmed by an ROI RFX analysis. These results corroborate and extend earlier findings by de Zubicaray et al. (2002), who observed similar effects in STG in a pictureword interference paradigm. They too, observed higher BOLD-signal changes when participants named pictures while ignoring superimposed unrelated words, compared to related words. In addition, we observed such effects in posterior IFG, which has also been implicated in phonological processing. Although it is often assumed that words are automatically processed or that they capture attention briefly even if they are supposed to be ignored (Stroop, 1935), the current findings, show that phonological processing can also take place for ignored picture stimuli.

Behavioural indications of ignored picture processing have previously been found in a picture-picture interference paradigm, in which participants name one of two superimposed pictures, while ignoring the other. Faster reaction times were observed when the names of the pictures are phonologically related compared to unrelated trials (Morsella \& Miozzo, 2002; Navarrete \& Costa, 2005) ${ }^{3}$. The present study is the first to show effects of ignored picture processing in an fMRI paradigm, implying that under these circumstances, a distractor picture cannot totally be ignored. In fact, distractor processing does not stop at visual processing areas, but proceeds well into the language system. In addition, the systematic variation of phonological relatedness and the observed effects clearly showed that the phonological codes of the distractor picture names were activated.

Our initial expectation was that the phonological relatedness effect should be stronger in the onset task than in the offset task, as the relatedness manipulation in the material concerned the onset phonemes only. But contrary to our predictions, we did not observe any significant relatedness effects in the onset but a robust effect in the offset monitoring task. Possibly, the absence of the onset effect can be explained by the fact that in order to perform this task, only the first letter of a picture's name needed to be generated. The production of a word's phonological code is believed to occur in an incremental manner, that is, from left to right (Meyer, 1990; 1991). If name generation stops or goes unmonitored after generation of the first letter, any overlap between the name of the target picture and that of the distractor may go unnoticed, or at least does not lead to reliable differences compared to the unrelated condition. The offset monitoring task, on the other hand, requires the entire name of a picture to be activated and monitored in order to

3 In two studies trying to reproduce these behavioural effects with Dutch stimuli, we failed to observe phonological facilitation effects with the exact same experimental parameters. 
identify the last phoneme. In this case, the name of the picture, including all phonemes in which overlap with the distractor name exists, are more thoroughly processed, which may lead to an increased detection rate of the relatedness manipulation by phonological processing areas. This interpretation implies that the phonological properties of distractor items are only activated, or at least their overlap with that of the target item only detected, when the task involves scrutinous monitoring of those properties. This implication is supported by the fact that no phonological relatedness effects were observed in IFG the semantic judgment task, in which it is not necessary to produce the name of the target picture.

Unexpectedly, however, we also observed a phonological relatedness effect in the control task (bar-width comparison). This effect was primarily observed in the anterior parts of the left insula. Interestingly, the relatedness effect in this area is reversed compared to the one observed in the offset task. That is, blocks with phonological related picture pairs led to higher activation than unrelated blocks. These differences cannot be explained in terms of stimulus differences between related and unrelated conditions for two reasons. First, each picture was repeated equally often in related and unrelated conditions and at the same position. Second, since the exact same picture combinations were used across the tasks, any effects of the pairing of the stimuli should also have appeared in the linguistic tasks. We will discuss the finding of a relatedness effect in light of insular functions in the section below.

\section{Hemispheric lateralisation and task-specific activations}

In line with previous observations that in right-handed people, language processing is lateralised mainly towards the left hemisphere (Frost et al., 1999; Indefrey \& Levelt, 2004), the linguistic tasks activated an extensive, left-hemispheric network compared to the control task. This network included the left MTG (semantic processing) and STS/STG (phonological processing), and the left insula, IFG and MFG (semantic/phonological processing).

It has been suggested in the literature that the IFG plays a role in both phonological and semantic processing, but that the anterior part (BA 45/47) is specialised in semantic processing, whereas the posterior part (BA 44/45) plays an important role in phonological processing (e.g. Bookheimer, 2002; Fiez, 1997; Hagoort, 2005; Indefrey \& Levelt, 2004; Noesselt et al., 2003; Poldrack et al., 1999). Corroborating previous studies, we observed that phoneme monitoring activated the posterior part of IFG stronger than semantic categorisation, whereas the reverse was true for the anterior part of the IFG. Noesselt et al. (2003) proposed that the posterior part of IFG may be specialised for phonological processing, and that this is automatically activated in semantic tasks. In line with this proposition, we observed significant activation increase in this area in the semantic task 
compared to the control task, indicating that phonological processing was taking place during semantic categorisation. However, the fact that this activation is consistently lower than that in the phonological task, indicates that perhaps phonological processing is not as thorough in a semantic categorisation task, which could explain the absence of a relatedness effect in this condition. In addition, the semantic task significantly activated a large portion of the MTG stronger than the phonological task. MTG has been associated with the storage of long-term semantic memory, whereas the anterior IFG is believed to involve selection and control from that memory (Cabeza \& Nyberg, 2000; Fiez, 1997). Therefore, although these areas are activated in phonological tasks (semantic retrieval takes place before the phonological code is activated (Schmitt et al., 2000; van Turennout et al., 1997), the semantic task requires an active judgement of the category membership of the picture. This requires stronger selective processes, leading to the higher activation of these areas.

The role of the insula in language processing is less clear. Insular regions have been associated with several kinds of aphasia, including Broca's and Wernicke's aphasia, indicating it's role in phonological processing. The anterior part of the insula was recently found to be susceptible to sublexical spelling-to-sound processing (Borowsky et al., 2006), which would explain the higher activation in the phonological tasks compared to the semantic task. However, the insula was just as active in the control task as in the phonological tasks. In addition, a phonological relatedness effect was observed in this area. This implies that the names of both pictures were phonologically processed in the control task. Perhaps the control task (bar-width discrimination) was not attentionally demanding enough to fully engage participants' attention. Residual resources may have been employed to process the pictures, while the insula monitored the results of this attentional spill-over. Data from a pilot behavioural experiment indicated that reaction times severely increased when participants performed the control task and the onset monitoring task simultaneously compared to the single tasks, but that performing both tasks was possible (see footnote 2). Thus, although the control task was attentionally demanding, we cannot exclude the possibility of attentional resources spilling over to the processing of the pictures.

68 The absence of phonological relatedness effects in the insula in linguistic tasks implies that this monitoring only took place in non-linguistic tasks (perhaps scanning the environment for stimuli with linguistic content) or when task demands allowed additional resources to be diverted to distractor processing. In the insula, related picture pairs were associated with higher activation levels than unrelated ones (i.e. the reverse pattern from that observed in the IFG). This implies that perhaps one feature of this monitoring process is the detection of salient features of linguistic stimuli, in this case the phonological relatedness between two peripherally presented stimuli. In this respect, it would be interesting to observe whether the insula is also involved in detecting salient stimuli in an 
unattended auditory stream, as occurs the cocktail party effect (Moray, 1959; Wood \& Cowan, 1995). However, due to the unexpected observation of relatedness effects in the insula, the absence of such effects in the linguistic tasks, and the poorly understood role of the insula in language processing, additional research is required to elucidate its role in the processing and monitoring of ignored stimuli.

In summary, this study is the first to show that phonological properties of ignored pictures can be processed by phonological processing areas such as Wernicke's area and the posterior IFG. These results implicate that ignored pictures can be identified beyond their physical properties. Since effects of phonological relatedness were not observed in all task conditions, we suggest that the depth of processing and the properties of a picture that are identified depend on task demands. Whereas we addressed the identification of phonological properties of ignored pictures, future studies may address relatedness effects with other stimulus features, such as semantics.

\section{Acknowledgements}

The authors wish to thank Alard Roebroeck and Jochen Seitz for valuable discussion and Sven Gijssen and Armin Heinecke for technical support. This project was supported by NWO ASPASIA grant nr. 015.001.053 to B.M. Jansma (Schmitt). 


\section{References}

Beck, D. M., \& Kastner, S. (2005). Stimulus context modulates competition in human extrastriate cortex. Nat Neurosci 8, $1110-1116$.

Bentley, P., Vuilleumier, P., Thiel, C. M., Driver, J., \& Dolan, R. J. (2003). Effects of attention and emotion on repetition priming and their modulation by cholinergic enhancement. J Neurophysiol 90, 1171-1181.

Bles, M., Schwarzbach, J., De Weerd, P., Goebel, R., \& Jansma, B. M. (2006). Receptive field size-dependent attention effects in simultaneously presented stimulus displays. Neuroimage 30, 506-511.

Bookheimer, S. (2002). Functional MRI of language: new approaches to understanding the cortical organization of semantic processing. Annu Rev Neurosci 25, 151 188.

Borowsky, R., Cummine, J., Owen, W. J., Friesen, C. K., Shih, F., \& Sarty, G. E. (2006). FMRI of ventral and dorsal processing streams in basic reading processes: insular sensitivity to phonology. Brain Topogr 18, 233-239.

Bradshaw, J. L. (1974). Peripherall presented and unreported words may bias the perceived meaning of a centrally fixated homograph. Journal of Experimental Psychology 103.

Broadbent, D. (1958). Perception and communication (London, Pergamon Press).

Cabeza, R., \& Nyberg, L. (2000). Neural bases of learning and memory: functional neuroimaging evidence. Curr Opin Neurol 13, 415-421.

Chelazzi, L. (1999). Serial attention mechanisms in visual search: a critical look at the evidence. Psychol Res 62, 195-219.

Cherry, E. C. (1953). Some experiments on the recognition of speech, with one and with two ears. The Journal of the Acoustical Society of America 25, 975-979.

de Zubicaray, G. I., McMahon, K. L., Eastburn, M. M., \&Wilson, S. J. (2002). Orthographic/ phonological facilitation of naming responses in the picture-word task: an eventrelated fMRI study using overt vocal responding. Neuroimage 16, 1084-1093.

Desimone, R. (1998). Visual attention mediated by biased competition in extrastriate visual cortex. Philos Trans R Soc Lond B Biol Sci 353, 1245-1255.

Duncan, J. (2006). EPS Mid-Career Award 2004: brain mechanisms of attention. Q J Exp Psychol (Colchester) 59, 2-27.

Eger, E., Henson, R. N., Driver, J., \& Dolan, R. J. (2004). BOLD repetition decreases in object-responsive ventral visual areas depend on spatial attention. J Neurophysiol 92, 1241-1247. 
Eriksen, B. A., \& Eriksen, C. W. (1974). Effects of noise letters upon the identification of a target letter in a nonsearch task. Perception \& Psychophysics 16, 143-149.

Fiez, J. A. (1997). Phonology, semantics, and the role of the left inferior prefrontal cortex. Hum Brain Mapp 5, 79-83.

Frost, J. A., Binder, J. R., Springer, J. A., Hammeke, T. A., Bellgowan, P. S., Rao, S. M., \& Cox, R. W. (1999). Language processing is strongly left lateralized in both sexes. Evidence from functional MRI. Brain 122 ( Pt 2), 199-208.

Genovese, C. R., Lazar, N. A., \& Nichols, T. (2002). Thresholding of statistical maps in functional neuroimaging using the false discovery rate. Neuroimage 15, 870 878.

Goebel, R., Muckli, L., \& Kim, D.-S. (2003). The Visual System. In: G. Paxinos, and J. K. Mai, (Eds.), The Human Nervous System. New York, Academic Press.

Grill-Spector, K., Kushnir, T., Edelman, S., Itzchak, Y., \& Malach, R. (1998). Cue-invariant activation in object-related areas of the human occipital lobe. Neuron 21, 191 202.

Grill-Spector, K., Kourtzi, Z., \& Kanwisher, N. (2001). The lateral occipital complex and its role in object recognition. Vision Res 41, 1409-1422.

Hagoort, P. (2005). On Broca, brain, and binding: a new framework. Trends Cogn Sci 9, 416-423.

Indefrey, P., \& Levelt, W. J. (2004). The spatial and temporal signatures of word production components. Cognition 92, 101-144.

Jescheniak, J. D., \& Schriefers, H. (2001). Priming effects from phonologically related distractors in picture-word interference. Q J Exp Psychol A 54, 371-382.

Kastner, S., De Weerd, P., Desimone, R., \& Ungerleider, L. (1998). Mechanisms of Directed Attention in the Human Extrastriate Cortex as Revealed by Functional MRI. Science 282, 108-111.

Kastner, S., Pinsk, M. A., De Weerd, P., Desimone, R., \& Ungerleider, L. (1999). Increased Activity in Human Visual Cortex during Directed Attention in the Absence of Visual Stimulation. Neuron 22, 751-761.

Kastner, S., De Weerd, P., Pinsk, M. A., Elizondo, M. I., Desimone, R., \& Ungerleider, L. (2001a). Modulation of Sensory Suppression: Implications for Receptive Field Sizes in the Human Visual Cortex. Journal of Neurophysiology 86, 1398-1411.

Kastner, S., \& Ungerleider, L. (2001 b). The neural basis of biased competition in human visual cortex. Neuropsychologia 39, 1263-1276.

Lavie, N. (1995). Perceptual load as a necessary condition for selective attention. J Exp Psychol Hum Percept Perform 21, 451 -468. 
Lavie, N., Hirst, A., de Fockert, J. W., \& Viding, E. (2004). Load theory of selective attention and cognitive control. J Exp Psychol Gen 133, 339-354.

Meyer, A. S. (1990). The time course of phonological encoding in language production: The encoding of successive syllables. Journal of Memory and Language 29, 524 545.

Meyer, A. S. (1991). The time course of phonological encoding in language production: Phonological encoding inside a syllable. Journal of Memory and Language 30, 69-89.

Moray, N. (1959). Attention in dichotic listening: Affective cues and the influence of instructions. Quarterly Journal of Experimental Psychology 11.

Morsella, E., \& Miozzo, M. (2002). Evidence for a cascade model of lexical access in speech production. J Exp Psychol Learn Mem Cogn 28, 555-563.

Murray, S. O., \& Woiciulik, E. (2004). Attention increases neural selectivity in the human lateral occipital complex. Nat Neurosci 7, 70-74.

Navarrete, E., \& Costa, A. (2005). Phonological activation of ignored pictures: Further evidence for a cascade model of lexical access. Journal of Memory and Language 53, 359-377.

Noesselt, T., Shah, N. J., \& Jancke, L. (2003). Top-down and bottom-up modulation of language related areas--an fMRI study. BMC Neurosci 4, 13.

Peterson, R. R., \& Savoy, P. (1998). Lexical selection and phonological encoding during language production: evidence for cascaded processing. Journal of Experimental Psychology: Learning, Memory, and Cognition 24, 539-557.

Pinsk, M. A., Kastner, S., Desimone, R., \& Ungerleider, L. (1999). An estimate of receptive field sizes in human visual cortex. Neurolmage 9, s885.

Poldrack, R. A., Wagner, A. D., Prull, M. W., Desmond, J. E., Glover, G. H., \& Gabrieli, J. D. (1999). Functional specialization for semantic and phonological processing in the left inferior prefrontal cortex. Neuroimage 10, 15-35.

Rees, G., Russell, C., Frith, C. D., \& Driver, J. (1999). Inattentional blindness versus inattentional amnesia for fixated but ignored words. Science 286, 2504-2507.

Ruz, M., Worden, M. S., Tudela, P., \& McCandliss, B. D. (2005). Inattentional amnesia to words in a high attentional load task. J Cogn Neurosci 17, 768-776.

Schmitt, B. M., Munte, T. F., \& Kutas, M. (2000). Electrophysiological estimates of the time course of semantic and phonological encoding during implicit picture naming. Psychophysiology 37, 473-484.

Sereno, M. I., Dale, A. M., Reppas, J. B., Kwong, K. K., Belliveau, J. W., Brady, T. J., Rosen, B. R., \& Tootell, R. B. H. (1995). Borders of multiple visual areas in human revealed by functional magnetic resonance imaging. Science 268, 889-893. 
Sperling, G. (1960). The information available in brief visual presentations. Psychological Monographs: General and Applied 74, 1-28.

Starreveld, P. A., \& La Heii, W. (1996). Time-course analysis of semantic and orthographiccontext effects in picture naming. Journal of Experimental Psychology: Learning, Memory, and Cognition 22, 896-918.

Stroop, J. R. (1935). Studies of interference in serial verbal reactions. Journal of Experimental Psychology 18, 643-662.

Talairach, J., \& Tournoux, P. (1988). Co-planar stereotaxic atlas of the human brain (New York (NY), Thieme).

van Turennout, M., Hagoort, P., \& Brown, C. M. (1997). Electrophysiological evidence on the time course of semantic and phonological processes in speech production. J Exp Psychol Learn Mem Cogn 23, 787-806.

Vogel, E. K., Woodman, G. F., \& Luck, S. J. (2005). Pushing around the locus of selection: evidence for the flexible-selection hypothesis. J Cogn Neurosci 17, 1907-1922.

Wood, N., \& Cowan, N. (1995). The cocktail party phenomenon revisited: how frequent are attention shifts to one's name in an irrelevant auditory channel? J Exp Psychol Learn Mem Cogn 21, 255-260.

Yi, D. J., Woodman, G. F., Widders, D., Marois, R., \& Chun, M. M. (2004). Neural fate of ignored stimuli: dissociable effects of perceptual and working memory load. Nat Neurosci 7, 992-996.

Yi, D. J., Kelley, T. A., Marois, R., \& Chun, M. M. (2006). Attentional modulation of repetition attenuation is anatomically dissociable for scenes and faces. Brain Res $1080,53-62$. 


\section{Appendix 4.1}

1. banaan - ballon

2. bord - bom

3. fabriek - fagot

4. fazant - fakkel

5. galg - gans

6. gitar - gieter

7. hamster - harpoen

8. hand - hark

9. kaas - kaart

10. kerk - kers

11. ladder - lasso

12. liniaal - libel

13. map - maïs

14. matras - masker

15. pion - pistool

16. paraplu - paprika

17. raket - radio

18. rok - rolstoel

19. tong - tor

20. trap - tram

21. wieg - wiel

22. worst - wolk

23. vos - vork

24. vulkaan - vulpen 


\section{$\bigcirc$}

O

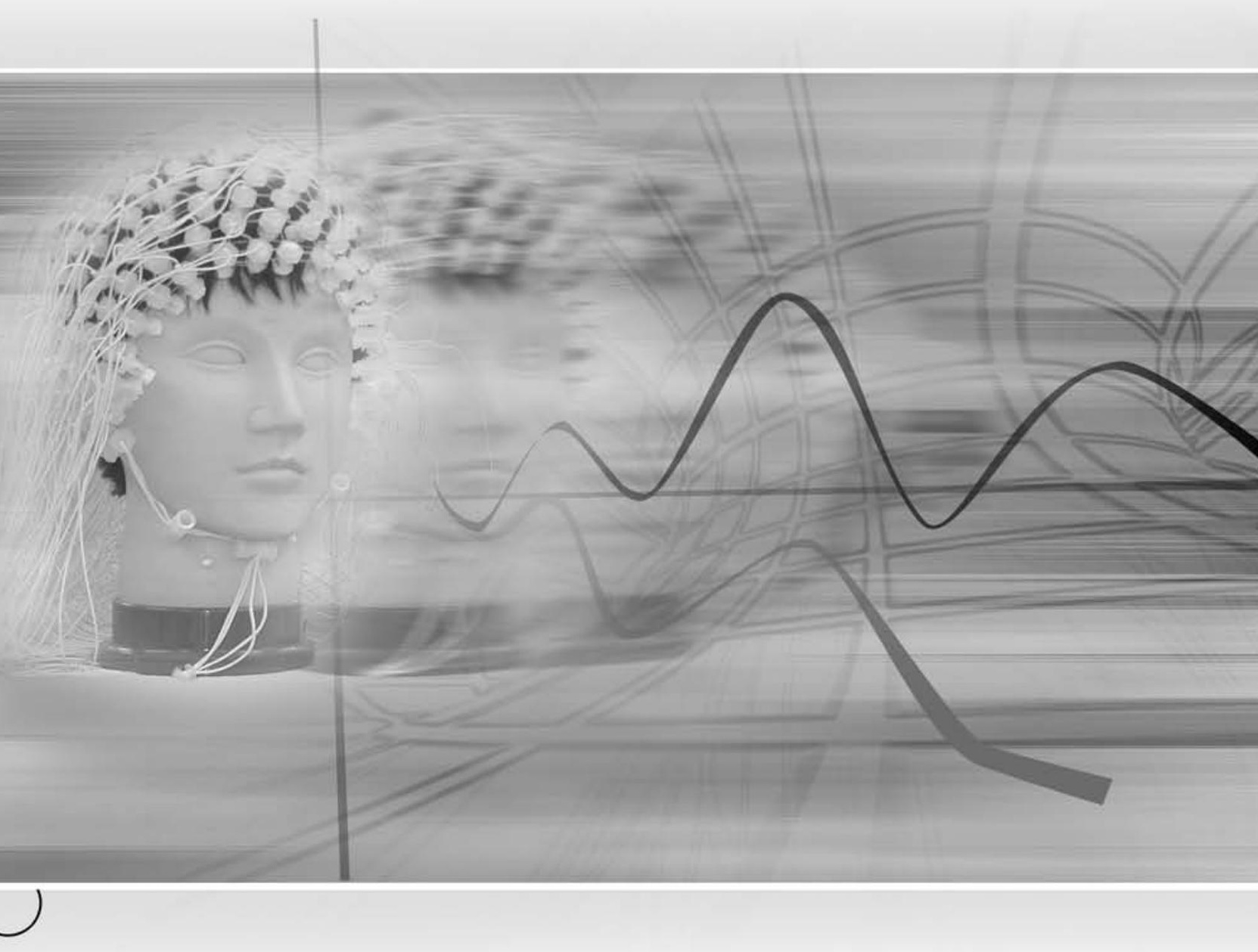

○ 


\section{Neural aspects of cohort-size reduction during visual gating}

In psycholinguistics, the coactivation of lexical candidates by partial input has received a lot of attention. More recently, psychophysiological studies have shed more light on this issue and identified two possible time windows in which effects of coactivation of lexical candidates can be observed in event-related potentials (ERPs). However, these studies cannot be used to disentangle effects of coactivation effects from the inhibition of candidates that do not match input. A new visual gating paradigm is presented, in which stimuli were visually presented letter by letter, decreasing the size of the cohort of lexical candidates as more letters are presented. Stimuli were created such that at the letter position of interest, the amount of co-activated candidates was kept constant while manipulating the size of the reduction of the cohort. The resulting ERPs indicate two components (frontal P2, and a left temporal late negativity) that scaled with cohort-size reduction. These results show that a visual gating paradigm can be used to disentangle coactivation of lexical candidates from inhibition of non-matching items, and that these processes are closely related to each other in time.

Based on: Bles, M., Alink, A., \& Jansma, B.M. (2007). Neural aspects of cohort-size reduction during visual gating. Brain Research 1150, 143-154. 


\section{Introduction}

It is generally assumed that, in language comprehension, partial auditory or visual input activates cohorts of lexical candidates that match this input. With more incoming information, the initial set of candidates is reduced such that only matching items remain in the cohort. The reduction process terminates when one candidate remains, at which point word recognition takes place. Cognitive models of speech and written language comprehension deal with acoustic, graphic, phonetic and phonological encoding, accompanied by semantic and syntactic activation and discourse integration (e.g. Ans et al., 1998; Coltheart et al., 1991; Cutler \& Clifton, 1999; Gaskell \& Marslen-Wilson, 1997; Goldinger et al., 1992; Johnson \& Pugh, 1994; Marslen-Wilson, 1987; Masson, 1995; McClelland \& Elman, 1986; Norris et al., 1995; Perfetti, 1999). Such models have elaborated ideas about how the activation of the initial set and the selection of the winner actually takes place. These ideas differ between models, and there is still ongoing discussion about the details of word recognition. A consistent idea across the literature is that partial linguistic input can coactivate a set of several lexical items (hereafter called a 'cohort'). Coactivation of lexical items has received a lot of attention in psycholinguistics and more recently in the psychophysiological literature. To our knowledge, less work has been reported on the reduction of the cohort of activated items and the nature of the neural selection of the target word from this cohort. The goal of this paper is to focus on processes related to neural aspects of "cohort-size reduction" during word recognition.

Evidence for the coactivation of lexical candidates can be found in a variety of studies with different psycholinguistic paradigms. In cross-modal semantic priming studies, gated fragments of words such as captain are followed by a visually presented target word for lexical decision. The target word can be semantically related either to the spoken word itself (e.g. ship, semantically related to captain) or to a cohort competitor sharing the same initial sounds (e.g. guard, semantically related to captive). Typically, gated fragments like /kæpt/, that are consistent with both the actual word and its cohort competitor facilitate the processing of both the actual target and the cohort competitor compared to unrelated control targets (Zwitserlood, 1989). These results are consistent with the idea that initial form-based access activates lexical candidates and their semantic properties, allowing facilitation of a response to a semantically related word (Marslen-Wilson, 1987).

More recently, high temporal resolution methods such as eye- and hand-movement studies and event related potentials (ERP) have been used to gain more insight in the time course of competition between coactivated words. For example, a general finding in eye movement studies is that when participants view arrays of pictures while hearing a target word (in a neutral context), more fixations are made to cohort competitors of the target word than to phonologically unrelated distractors (e.g. Allopenna et al., 1998; Dahan et al., 2001; Dahan \& Tanenhaus, 2004). More precisely, the amount of fixations to cohort 
competitors starts to diverge from fixations to unrelated distractor words as early as 200 ms after word onset. Taking into account that eye movements need $150-180 \mathrm{~ms}$ of preparation time, these data indicate that lexical activation influences eye movements in the first 100 ms after word onset. Importantly, the number of fixations made to targets and to cohort competitors is equal until approximately 200 - 300 ms after enough information is present in the signal to disambiguate targets from cohort competitors, indicating that the lexical representations of both the targets and the cohort competitors are activated and considered by participants.

Similarly, in a study in which participants were required to make a PC mouse movement towards a picture depicting a spoken word, the trajectory of the mouse movement was found to be influenced by the presence of a cohort competitor (Spivey et al., 2005). The trajectory of the mouse followed an equidistant path to both pictures for a longer time when the distractor picture was a cohort competitor. This was interpreted as being consistent with "a description of two nearly equibiased attractors initially pulling the system toward their shared midpoint" (p 10394). Only when enough information was present in the speech signal to disambiguate the two stimuli, the target became a stronger attractor, pulling the movement of the mouse towards it.

Psychophysiological evidence that the meaning of several potential candidates is activated comes from a study by van Petten et al. (1999). In an ERP study, participants listened to sentences that ended with contextually congruous words, incongruous words that shared the same initial phonemes (cohort incongruous words), or words that rhymed with the congruous words (rhyme incongruous words). The target ERP component in this study was the N400, which is usually observed when participants are presented with semantically incongruous words in a sentence, indicating that it reflects semantic integration (Kutas \& Hillyard, 1980; Kutas \& Hillyard, 1984). The critical finding in this study was that the N400 amplitude for rhyme incongruous words deviated from that of congruous words and cohort incongruous words as early as $200 \mathrm{~ms}$ before the isolation point (i.e. the moment when enough information was present to have one lexical candidate left). Moreover, the N400 amplitudes of the congruous words and the cohort incongruous words diverged from each other at a later time point, namely after the isolation point. These data clearly indicated that the meaning of alternative lexical candidates was at least partially activated and that the moment of selection depended on the amount of onset-phonemes that were shared between target and competitor. These data have been replicated in a subsequent study by van den Brink et al. (2006), who also showed that the onset of the N400 effect occurs before the isolation point. Furthermore, the onset latency of the N400 was not influenced by the exact timing of the isolation point, which implies that semantic integration takes place before a unique word candidate has been selected.

In a cross-modal priming task, Friedrich (2005) showed that visual targets that are fully congruent with previously presented auditory primes lead to most negative ERP 
amplitudes at centro-temporal electrodes, followed by targets that were only partially congruent, and targets that were fully incongruent, respectively. The amplitude differences caused by prime-target congruency were reflected in a P350 component which peaked at left-hemispheric scalp sites at around $350 \mathrm{~ms}$. Based on these results and those of a previous study in which the P350 was also observed in a unimodal priming task (Friedrich et al., 2004), the P350 was interpreted as an index for the activation of matching words in a modality-independent mental lexicon. In this respect, the P350 shares similarities with the M350 component observed in MEG studies (e.g. Pylkkanen et al., 2002; Pylkkanen et al., 2004; Pylkkanen et al., 2006; Stockall et al., 2004). The latency of the M350 has been found to vary with phonotactic probability of written words (Pylkkanen et al., 2002; Stockall et al., 2004). Words with high phonotactic probabilities lead to lower M350 latencies than words with low probabilities. Similar to the previously mentioned P350, the M350 was interpreted to reflect lexical activation, and the decreased latency for highprobability words was a result of facilitated lexical activation.

Further evidence for the activation of multiple lexical candidates in written words came from an ERP study by Holcomb et al. (2002). Participants read words and pseudowords that belonged to small or large lexical neighbourhoods while performing either a lexical decision task or a semantic categorization task. The authors found an enhanced N400 component for both words and pseudowords with a high amount of orthographic neighbours. This N400 pattern was interpreted as supportive for the view that printed strings belonging to large neighbourhoods lead to increased levels of activation at either the form representation level, or the semantic representation level. Interestingly, in the semantic categorization task, effects of neighbourhood size could already be observed in the 150 - 350 ms time window. Along similar lines, Barber et al. (2004) and Hutzler et

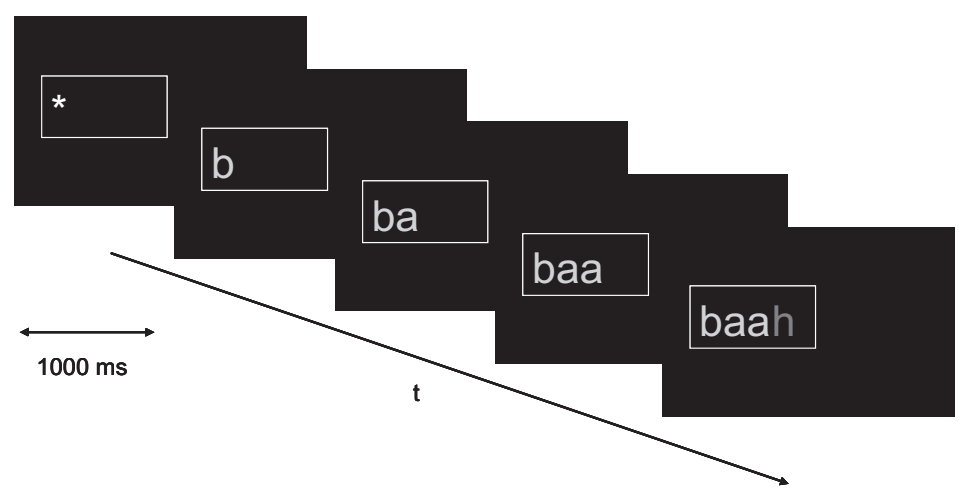


al. (2004) reported an N400 component varying with syllable frequency (higher N400 for high syllable frequency stimuli), supporting the idea that high frequency syllables trigger the activity of many lexical candidates since they are shared by many words. In addition to the $\mathrm{N} 400$ effect, and similar to Holcomb et al. (2002), an early negativity for high frequency syllables was observed 150 ms (Barber et al., 2004) or 190 ms (Hutzler et al., 2004) after presentation of the stimulus. The interpretation of these results was that early first-syllable frequency effects may be associated with pre-lexical processes like phonological stimulus processing and activation of lexical candidates. In addition, Barber et al. (2004) hypothesize that the subsequent N400 effect might not just reflect differences in the activation of lexical candidates, but might also reflect differences in inhibition between lexical candidates, associated with selecting the proper candidates from many activated items.

In summary, there is general empirical evidence and consensus that speech or written input activates several initial lexical candidates matching input. For recognition to take
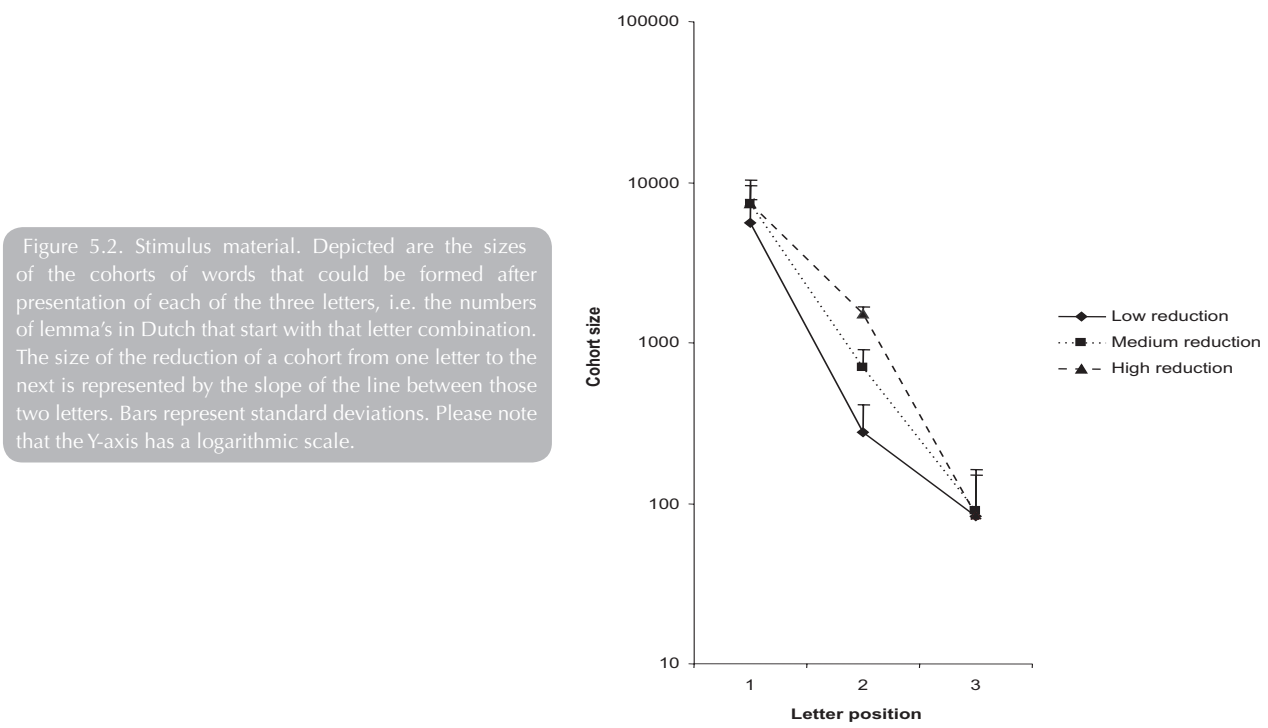

place, it is necessary to narrow this initial set of candidates down to a single, best-fitting match. Contrary to support for coactivation of lexical candidates, the processes involved in selecting appropriate candidates has not been investigated so far. In the present study, we applied a paradigm that enables us to look at the selection process itself, rather than the activation of candidates. In a visual gating paradigm we presented sets of three letter combinations, letter by letter (see Figure 5.1).

For each set, we calculated beforehand how many lemma's in the Dutch language start with the first, the first two or all three letters of the set (CELEX database, Baayen et 
al., 1995). As displayed in Figure 5.2, after the first letter, the cohort size (i.e. the amount of words that start with that letter) was large, the second letter reduced that size and differentiated stimuli into three types of cohorts (relatively large, medium, small), the third letter reduced the cohort size of all three conditions to the same size.

After presentation of the third letter, the amount of lexical candidates that matches current input was equal for all three groups of stimuli. However, the reduction of the cohort size (i.e. the slope that can be observed in figure 5.2) from the second to the third letter position differs between conditions. The rationale behind this design is that any ERP difference in response to the third letter presentation cannot be explained by the size of currently active cohorts, as they are similar across conditions from the onset of the third letter onwards. Rather, a variation in the ERP should reflect the selection processes that are involved in the reduction of the size of the active cohort.

The letter positions were marked in the EEG signal and the ERP signal in response to the third letter was analyzed in order to observe the reduction process at a millisecond scale. Assuming that inhibition of non-matching lexical candidates occurs at approximately the same time as the activation of the candidate set, we expected to observe effects of cohort-size reduction in similar time windows as reported for (pre-)lexical coactivation (Barber et al., 2004; Carreiras et al., 2005; Holcomb et al., 2002; Hutzler et al., 2004). Specifically, the P2 and N400 components were expected to display effects of cohort-size reduction.

\section{Methods}

\section{Partic ipants}

Twenty-two right-handed native speakers of Dutch participated in this study. They were paid for their time. All were neurologically healthy and had normal or corrected to normal vision. Data of six participants had to be discarded due to excessive amounts of

82 eye movement artefacts (more than $25 \%$ of trials), or technical problems. The data of the remaining 16 participants ( 5 females, mean age 22.8 years) were analysed. This research project was approved by the ethical committee of the Faculty of Psychology, Maastricht University. 


\section{Stimuli}

In order to keep frequency and cohort size constant, while manipulating cohortsize reduction, three groups of 36 three-letter combinations were created. For each of these three-letter combinations, the amount of lexical candidates (lemma's) in the Dutch language that, start with the first, the first two, or all three letters were calculated (Celex database for lemma frequencies Baayen et al., 1995). For example, Dutch has over 5300 lemmas that start with the letter ' $h$ ', about 1500 lemmas starting with 'ho', and 200 candidates for 'hon'. Based on these stimulus properties, three groups of stimuli were created that differed in the size of the cohort of possible alternatives after presentation of a new letter (see figure 5.2). Groups were created in such a way that the average cohort size after presentation of the first letter was 5593 (standard deviation 2961), 7347 (3017) and 7356 (2153), for the three groups. More importantly, after presentation of the second letter, these cohorts were reduced to 276 (97), 704 (208) and 1526 (139) words for the three groups, labelled Low, Medium and High, respectively. The critical factor in the design was the equal size of the cohorts after the presentation of the third letter; 83 (60), 90 (73) and 93 (65), respectively. Based on this material, the size of the reduction of cohort size (i.e. cohort size after the second letter minus cohort size after the third letter) differentiates the three groups. I.e. the size of the reduction was 193 for the Low condition, 614 for the Medium condition, and 1442 for the High condition. To control for frequency effects, the average frequency of each of the members of the cohorts was matched for two-letter combinations (122 (84), 130 (63), 131 (90), respectively) and for three-letter combinations (103 (84), 98 (68), 102 (88), respectively).

Letters were presented in green (RGB $0 ; 255 ; 0$ ) or in red (RGB 255; 0 ; 0 , target for the reaction task of the participant, see below). Font type was courier, letter size was $0.5^{\circ}$ of visual angle. Letters were sequentially presented on a black background. The first letter appeared at the location of the fixation cross, subsequent letters were presented at $0.6^{\circ}$ of visual angle to the right of the previous letter, with a maximum of seven letters. The letters on the screen never formed a real word, and the red letter never appeared in the first three letters of an experimental trial. In addition to potential word segments, filler letter sequences were included that were presented in the same manner as the relevant strings, except that they were created by randomly generating strings of letters. These filler trials were included to distract participants from the fact that many of the stimuli formed parts of words, in order to reduce anticipation effects for word meaning in incoming strings. Furthermore, in these filler trials targets could also appear at the first 3 letter positions, which insured participants' attention to all letter positions. 


\section{Procedure}

Participants were told that they participated in a simple reaction time experiment. After explanation of the task, and application of the electrodes, participants were comfortably seated in an electrically shielded, sound-attenuated room in front of a computer monitor. They were told to keep the amount of movement as low as possible, and to be as fast and accurate as possible on the reaction task. On the monitor, a black screen with a white box was projected. In this box, a fixation cross appeared for a variable amount of time (1900$2500 \mathrm{~ms}$ ), to which participants were asked to fixate. A trial sequence reflecting the letterby-letter gating paradigm is displayed in figure 5.1. A trial started with the disappearance of the fixation cross, which was substituted by a green letter. At a rate of $1 \mathrm{~Hz}$, letters were added to the right of this letter until a red letter appeared. The participants' task was to monitor the colour of the newly presented letters, and press a response button on a response box with their right index finger whenever a red letter appeared, which ended the trial. A fixation cross reappeared until a new trial started. The 108 experimental stimuli (36 trigram trials $\times 3$ conditions) were randomly alternated with 54 filler stimuli. This block of stimuli was repeated three times per participant, leading to a total of 108 trials per condition.

\section{EEG recording}

The electroencephalogram (EEG) was measured using 29 tin electrodes mounted in an elastic cap (QuickCap, Neuroscan Inc. Tm). The electrodes were positioned according to the international 10-20 system (Jasper, 1958). Electrodes Fz, F3, F4, F7, F8, FCz, FC3, FC4, FP1 , FP2, AFz, T3, T4, T5, T6, TP7, TP8, Cz, C3, C4, CPz, CP3, CP4, Pz, P3, $\mathrm{P} 4, \mathrm{Oz}, \mathrm{O} 1, \mathrm{O} 2$ were measured as active leads. Scalp sites were online referenced to an electrode placed at the left mastoid (A1). The signal of an additional electrode placed at the right mastoid (A2), referenced to $A 1$, was later used for offline re-referencing of the signal to the average of $A 1 / A 2$. Vertical eye movements and blinks were measured with a bipolar montage placed above and below the left upper and lower orbital ridge. Horizontal eye movements were recorded with a bipolar montage placed at the left and right canthi. All electrode impedances were kept below $5 \mathrm{KOhm}$. The EEG was amplified (Synamp, Neuroscan Inc.) with a $.05-30 \mathrm{~Hz}$ bandpass and sampled at $2.5 \mathrm{~ms}$ intervals $(400 \mathrm{~Hz})$ and stored to disk. Epochs of $800 \mathrm{~ms}$ were created, time-locked to the onset of the third letter in each trial. Epochs included a $200 \mathrm{~ms}$ pre-stimulus baseline-correction interval and they were 1-30 Hz bandpass filtered (Butterworth, zero phase, 24dB/oct). After removal of eye blinks and other artifacts (on average $10 \%$ of the data, no difference between conditions), ERPs were calculated separately for each participant and each condition. 


\section{Results}

\section{Behavioural findings}

Data reported here belong to the 16 participants that were used in the ERP analysis. Behavioural data of 2 participants had to be discarded due to technical problems. Inspection showed that reaction times linearly decreased with letter position. Reaction times to filler trials with the target at position 1 were $584 \mathrm{~ms}$, whereas responses to targets at position 7 took only $369 \mathrm{~ms}$. This effect of target position in filler trials was highly significant $(F(6,78)=77.76, p<.001)$. A linear within-subjects contrast confirmed the strong linear decrease observed of reaction times as the letter position progressed $(F(1$, $13)=117.8, p<.001)$. Since target letters never appeared on letter positions 1 to 3 in experimental trials, a comparison between experimental and filler conditions is only possible at positions 4 to 7 . An ANOVA with letter position (4, 5, 6 and 7) and condition (filler vs. experimental) revealed no differences between targets that were preceded by experimental trials and those preceded by filler trials $(F(1,13)=2.15, p>.1)$. Again, the effect of target position was highly significant $(F(3,39)=45.62, p<.001)$. There was no interaction between target position and condition $(F(3,39)=.50, p>.5)$.

Due to the simple nature of the task, little errors were made. The amount average amount of missed targets was $5 \%$ over conditions. An ANOVA on the amount of missed targets in the filler condition revealed no effect of target position $(F(6,78)=1.41, p>$ .1). Analysis of the last 4 letter positions revealed no difference between the amount of missed targets that were preceded by either experimental or filler trials $(F(1,13)=.173, p$ $>$.5). No effect of letter position was observed $(F(3,39)=.53, p>.5)$, nor was there an interaction between letter position and condition $(F(3,39)=.52, p>.5)$. The amount of false alarms was extremely small (on average $.5 \%$ ). There were no differences between experimental and filler conditions $(F(1,13)=1.53, p>.1)$ or letter position $(F(5,65)$ $=3.23, p>.05)$, nor was there a significant interaction between position and condition $(F(5,65)=.29, p>.5)$.

\section{Elec trophysiological findings}

\section{Third letter presentation}

The grand average waveforms of ERPs elicited by the third letter are presented in figure 5.3. All conditions showed an N1-P2 complex which was most prominent at anterior sites, followed by a large frontal negativity occurring between 300 and 500 ms after onset of the third letter. At posterior sites, the morphology of the ERPs was slightly different, 
probably due to the nature of our stimulus material (letter-by-letter gating). The posterior $\mathrm{N} 1$ component was slightly delayed, compared to the anterior N1 component, and was especially large at electrodes $\mathrm{T} 5$ and $\mathrm{O} 1$ (i.e. contra-lateral to the location of the newly presented letters). The subsequent P2 component was less pronounced than at anterior sites. Instead of the frontally visible negative component between 300 and $500 \mathrm{~ms}$, a few small negative and positive going deflections are observed at posterior sites.

For a reliable determination of time windows of interest, root mean quare (RMS) transformations (i.e. the square root of the mean of the squared potentials from each common-referenced electrode) were calculated (see figure 5.4). Note that these RMS plots were only used for visual selection of the time windows of interest. The subsequent statistical analysis was based on the mean amplitudes of the ERPs. Visual inspection of the RMS plots clearly indicated three time windows, especially at fronto-central sites, in which the parametric cohort-size manipulation resulted in systematic variations of the RMSand ERP amplitudes. The first of these time windows was related to the $\mathrm{N} 1$ component,
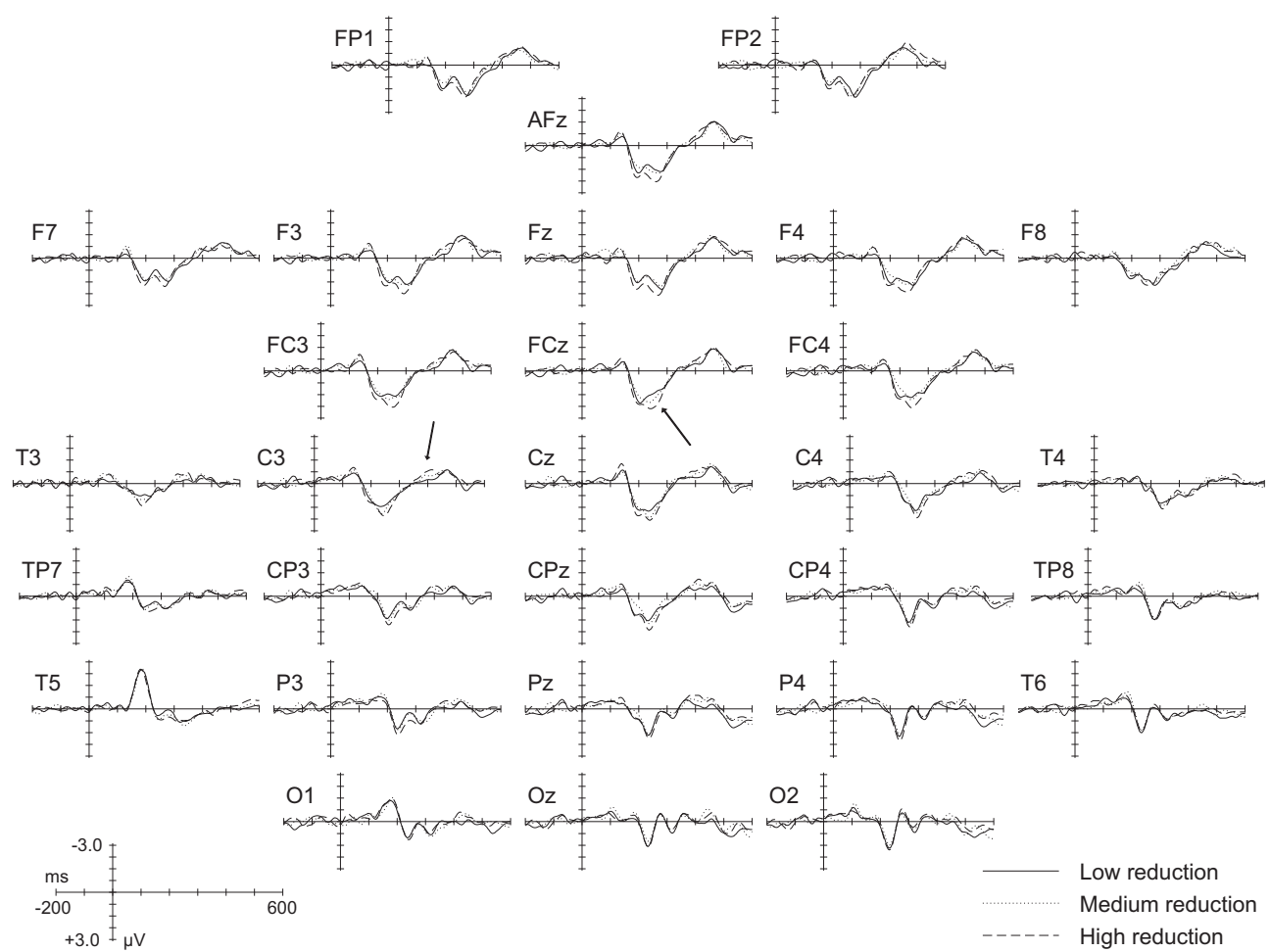

Low reduction Medium reduction High reduction 
between 124 and $164 \mathrm{~ms}$. The second time window of interest included the P2 component, between 212 and $280 \mathrm{~ms}$. Finally the window from 360 to $440 \mathrm{~ms}$ was selected for further analysis as it showed variation in the late negativity across conditions at central sites. Mean area amplitudes for each of these time windows were calculated for each condition for each participant, and were entered in a repeated measures General Linear Model (GLM) analysis with cohort-size reduction (3 levels: low, medium, high), laterality (3 levels: left ( $\mathrm{F} 3$ to $\mathrm{O} 1)$, middle ( $\mathrm{Fz}$ to $\mathrm{Oz}$ ), right ( $\mathrm{F} 4$ to $\mathrm{O} 2)$ ) and anterior-posterior (6 levels: $\mathrm{F}, \mathrm{FC}$, $\mathrm{C}, \mathrm{CP}, \mathrm{P}$ and $\mathrm{O}$ lines) as factors. All reported $\mathrm{p}$-values of tests with more than one degree of freedom in the numerator were Greenhouse-Geisser corrected to adjust for violations of the sphericity assumption (Greenhouse \& Geisser, 1959).

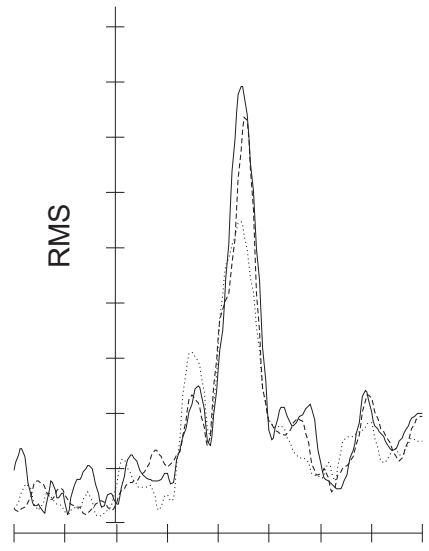

Position 1

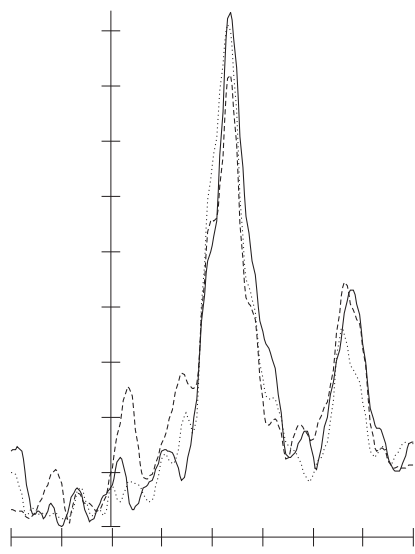

Position 2

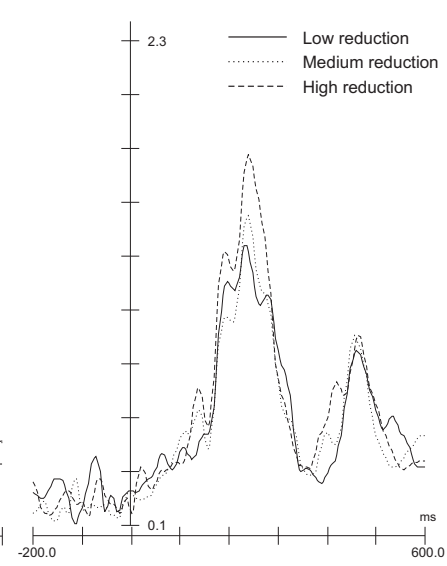

Position 3

Figure 5.4. RMS plots of each letter position. RMS plots were calculated by taking the square root of the average of the squared values of each of the 29 common-referenced electrodes. RMS values at the third letter position (rightmost panel) were used to define three time windows for further analysis: the first one ranging from $124-164 \mathrm{~ms}$, a second one from $212-280 \mathrm{~ms}$, and a third one from $360-400 \mathrm{~ms}$ All subsequent analyses were performed on the ERP values per participant per condition.

Analysis of the average ERPs in the $124-164$ ms time window showed no significant effects of cohort-size reduction $[F(2,30)=.71, p=.47]$. The effects of laterality $[F(2,30)$ $=2.06, p=.15]$ and anterior-posterior $[F(5,75)=.21, p=.70]$ were not significant either, nor were any of the interactions (all p-values $>.1$ ). Inspection of the single electrodes where the effect was most apparent visually (see figures 5.3 and 5.5) did not reveal any effects of cohort-size reduction in this time window (all p-values $>.1$ ).

For the 212 - $280 \mathrm{~ms}$ time window, the cohort-size reduction (3) * laterality (3) * anterior-posterior (6) analysis revealed a significant main effect of cohort-size reduction $[F(2,30)=3.66, p=.045]$. The most positive mean amplitudes were observed in the high reduction condition $(1.67 \mu \mathrm{V})$, followed by the medium $(1.39 \mu \mathrm{V})$ and low reduction conditions $(1.23 \mu \mathrm{V})$, respectively. The increasing mean area amplitude with increasing 

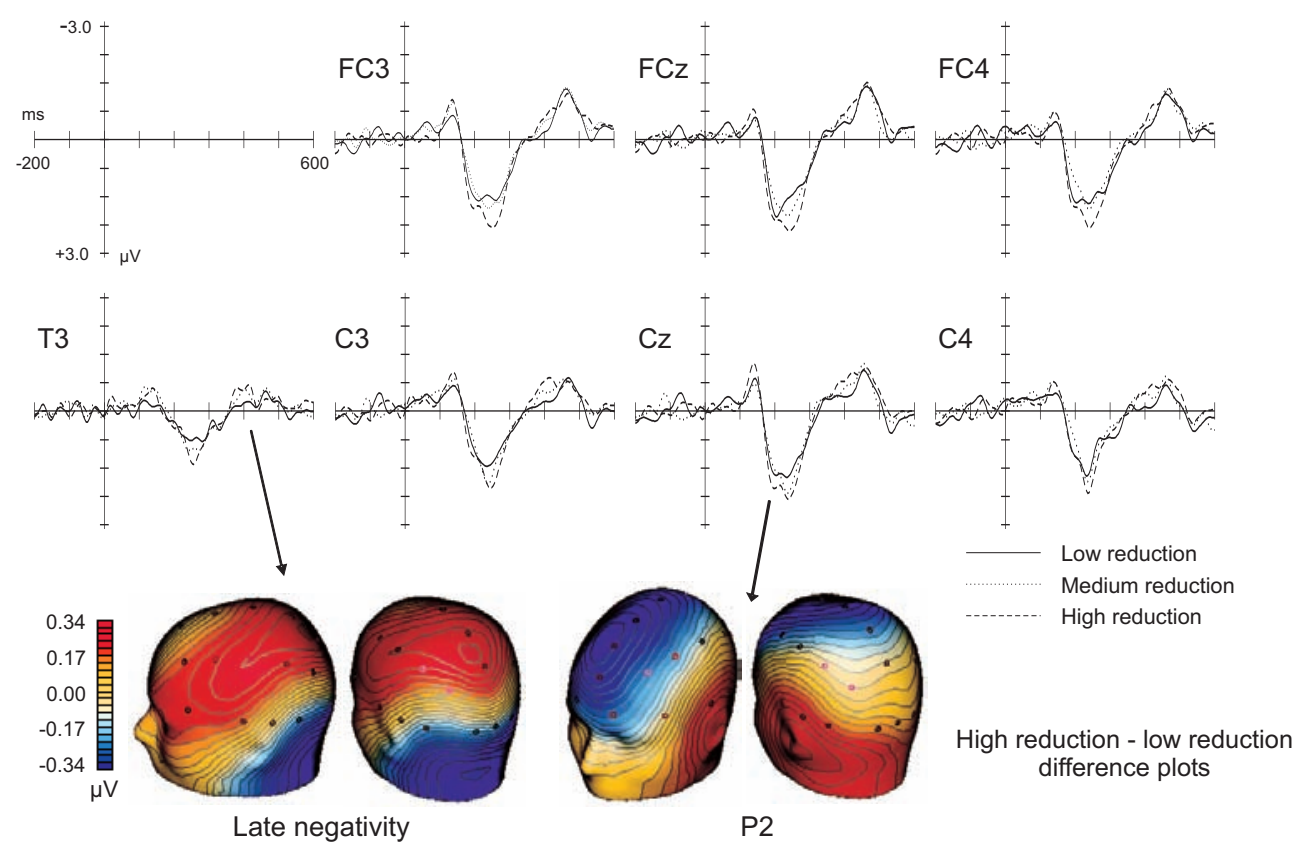

Figure 5.5. ERP waveforms and topographical difference maps in response to the presentation of the third letter. Waveforms are magnified versions of electrodes shown in figure 5.3 at which cohort-size reduction effects could be observed. Topographic maps represent the difference between the high reduction and low reduction conditions in the N400 time window (late negativity, left side) and the P2 time window (right side).

cohort-size reduction was confirmed by a significant linear within-subjects contrast for the reduction factor $[F(1,15)=5.24, p=.037]$, supporting the observation of a linear relationship between the experimental manipulation and ERP amplitude in this time window. A significant main effect of the anterior-posterior factor was observed $[F(4,60)=$ $21.84, p<.001]$. Mean amplitudes were largest over central sites and declined towards more anterior and posterior sites. The main effect of laterality was not significant $[F(2,30)$ $=1.93, p=.18]$. There was a statistical trend towards an interaction between cohort-size reduction $[F(10,150)=2.53, p=.064]$. Post-hoc analysis of the cohort-size reduction effect at the different anterior-posterior levels revealed that the effect was strongest at $F$ and FC lines, slowly declining towards more posterior sites. None of the other interactions were significant (all p-values $>.1$ ).

For the time window $360-440 \mathrm{~ms}$, a cohort-size reduction (3) x laterality (3) x anterior-posterior (6) GLM revealed a significant main effect of cohort-size reduction $[F(2,30)=3.89, p=.047]$. The most negative mean amplitudes were observed in the high reduction condition $(-.54 \mu \mathrm{V})$, followed by the medium $(-.35 \mu \mathrm{V})$ and low $(-.15 \mu \mathrm{V})$ conditions. Furthermore, a significant laterality effect was observed $[F(2,30)=6.65, p=$ 
.005), as well as a significant effect of the anterior-posterior factor $[F(4,60)=3.74, p=$ .038). Mean amplitudes were higher at midline electrodes than at more lateral electrodes and increased at more anterior sites. None of the interaction terms were significant (all $\mathrm{p}$-values $>$.05). The topography of the difference between the high and low reduction group in this time window (see figure 5.5) indicated a left central-temporal focus of the effect. The linear within-subjects contrast was significant $[F(1,15)=5.19, p=.038]$, confirming the linear relationship between cohort-size reduction and ERP amplitude in this time window at these scalp sites.

\section{First letter presentation}

In the 124 - 164 ms time window, no effects of stimulus condition were observed in response to the presentation of the first letter $[F(2,30)=2.79, p>.1]$. There was a significant effect of the anterior-posterior factor $[F(5,75)=6.61, p=.01]$; highest amplitudes were observed at FC and $C$ lines. The laterality effect was not significant, nor were any of the interactions (all p-values $>.1$ ).

In the 218-280 ms time window, a significant effect of condition was observed $[F(2,30)=5.20, p=.022]$. Most positive mean amplitudes were observed in the 'low reduction' condition $(2.02 \mu \mathrm{V})$, followed by the 'high' $(1.55 \mu \mathrm{V})$ and 'medium' (1.22 $\mu \mathrm{V})$ conditions. There was also a significant main effect of laterality $[F(2,30)=8.29, p=$ .002], with less positive mean amplitudes at right-hemisphere leads. No other main effects or interactions were observed in this time window (all $p$-values $>.1$ ).

In the $360-440$ ms time window, a significant laterality effect $[F(2,30)=11.19$, $p<.001]$ and an interaction of this factor with the anterior-posterior factor $[F(10,150)$ $=4.04, \mathrm{p}=.003 \mathrm{]}$ were observed. None of the other main factors or interaction terms approached significance (all p-values $>$.2).

\section{Second letter presentation}

In the 124 - 164 ms time window, there was a marginally significant effect of stimulus condition in response to the presentation of the second letter $[F(2,30)=3.44, p=.062]$. Amplitudes in the group of stimuli that had the highest reduction at letter position 3 were slightly higher than those of the other conditions. There was a main effect of laterality $[F(2,30)=4.08, p=.046]$, with higher amplitudes for left hemisphere electrodes. The unexpected effect of condition in this time window seemed mainly due to a problem in the baseline at left-lateralized electrodes for this condition, which is reflected by a significant condition * laterality interaction $[\mathrm{F}(4,60)=3.13, \mathrm{p}=.039]$. More detailed analysis confirmed that the effect of condition was only significant over left hemisphere leads $[F(2$, 
$30)=6.43, p=.008]$. At these leads, the 'high reduction' group differs from the other two conditions $(p<.05)$. No other significant interactions were observed in this time window.

In the $212-280$ ms time window, a significant effect of laterality $[F(2,30)=7.98$, $p<.002]$ and of the anterior-posterior factor $[F(5,75)=7.50, p=.012]$ were observed. There was no main effect of condition $[F(2,30)=.96, p>.25]$, nor were there any significant interactions (all $p$-values $>.25$ ).

In the $360-440 \mathrm{~ms}$ time window, a significant effect of condition was observed [F $(2,30)=3.66, p=.05]$. Most negative mean amplitudes were observed for the 'high reduction' $(-.35 \mu \mathrm{V})$ condition, followed by the 'medium' ( $-.07 \mu \mathrm{V})$ and 'low' $(.05 \mu \mathrm{V})$ conditions. The linear within-subjects contrast for this factor was significant as well $[F(1$, $15)=5.49, p=.033]$. Furthermore, there was a significant effect of laterality $[F(2,30)$ $=9.04, p=.001]$, with more negative mean amplitudes for central electrodes. There was also a significant interaction between laterality and the anterior-posterior factor $[F(10$, $150)=2.96, p=.02]$; the effect of laterality was slightly stronger at fronto-central electrode locations. None of the other factors or interactions approached significance (all p-values $>$.25).

\section{Disc ussion}

In this study, a new paradigm was applied to investigate neural processes of cohortsize reduction during word recognition. By visually presenting parts of words letter by letter at a slow rate during ERP recording, we were able to investigate parametrically manipulated cohort reduction online. The cohort-size reduction was manipulated by creating groups of stimuli in which the amount of lexical candidates that did not match the input after the presentation of the third letter differed between conditions. The aim of the study was to relate ERP effects to cohort reduction or selection processes proper. The potentially confounding factor of cohort size was controlled for by keeping it constant at the letter

90 position of interest (i.e. position three). Previous studies on cohort effects in language comprehension found evidence for a coactivation of lexical candidates both behaviourally (e.g. Allopenna et al., 1998; Dahan et al., 2001; Dahan \& Tanenhaus, 2004; MarslenWilson, 1987; Spivey et al., 2005; Zwitserlood, 1989) and electrophysiologically (Barber et al., 2004; Holcomb et al., 2002; Hutzler et al., 2004). The latter identified two ERP components of interest (the P2 and N400 components) whose amplitudes systematically varied with the amount of activated lexical items. Granted the assumption that coactivation of lexical candidates is closely related to the processes involved in inhibiting non-matching candidates we hypothesized that these ERP components are possible target components 
for detecting effects of cohort-size reduction as well. In fact, in the present study ERPs in response to the third letter scaled with cohort-size reduction in the time windows of the P2 and N400 components. In these time windows, higher ERP amplitudes were observed in conditions where cohort-size reduction was high and vice versa. Since higher ERP amplitudes are usually associated with higher neural processing loads (e.g. Hagen et al., 2006; Handy \& Mangun, 2000; Swaab et al., 2004; van Petten \& Luka, 2006) these data support the view that stronger inhibitive processes are required when larger groups of lexical candidates are discarded.

\section{P2 time wind ow}

Interestingly, effects of cohort-size reduction were observed as early as the frontal P2 component (212-280 ms), a time window in which orthographic neighbourhood size effects (Holcomb et al., 2002) and syllable frequency effects (Barber et al., 2004; Hutzler et al., 2004) have been reported. Our results confirm that the ERP in this window is also sensitive to the specific process of cohort reduction. The P2 effect observed in the preceding studies was interpreted as a precursor for the subsequent $\mathrm{N} 400$ effect, caused by a different amount of activated candidates. In our design, we dissociated the reduction of candidates from the amount of active candidates, and kept the amount of active lexical candidates identical across conditions after presentation of the third letter. Since we too observed a variation in P2 amplitude, we suggest that it reflects the amount of inhibition of words that mismatch phonological/orthographic input, rather than the amount of activated lexical candidates. The observed positive correlation between P2 amplitude and size of reduction implies that inhibition is stronger in situations where the cohort of activated lexical candidates has to be reduced to a larger degree.

This interpretation is supported by a variety of studies. In several different paradigms, frontal positive components have been identified that were involved in inhibitory and selective processes. For example, in the visual domain, a component variously named Frontal Selection Positivity (FSP), anterior P2 (P2a) or Frontal Positivity (FP) has been linked to stimulus evaluation and target selection (Kenemans et al., 1993; Potts, 2004; Smid et al., 1999), or to the suppression of irrelevant features (Guillem et al., 2001). In the auditory domain, the P2 component has been interpreted as being involved in the inhibition of distractors (Melara et al., 2002) and in target detection (Potts et al., 1998) . Although the exact nature of this P2 component is still under debate (see Potts, 2004), most authors stress its involvement in the evaluation of task relevance or in the inhibition of responses to distractor stimuli. The P2 component can be seen as reflecting inhibition and/or selection in a more general, modality independent manner. From the cited literature, the frontal positivity was reported as being sensitive to the selection of visual features like orientation, colour, shape, location and even faces. In addition, it has also been related to the selection 
of auditory stimuli, or - as reported here - to the linguistic reduction of a lexical cohort. Alternatively, a family of $\mathrm{P} 2 \mathrm{~s}$ could reflect very modality specific inhibition/selection. Depending on the type of information (visual, auditory, linguistic, or non-linguistic) one would expect slight variations in the P2 across tasks. The present study cannot give an answer on the functional specificity of the P2. Future studies are needed to systematically compare the P2 topography and the underlying neural generator/s across tasks. Most importantly, whether general or specific, the observed P2 in the present paradigm speaks to the time course of cohort reduction, which takes place in the time window $212-280$ ms after letter onset.

Recent studies have found evidence that lexical access is well underway between 100 and 200 ms after presentation of visual words (Hauk et al., 2006a; Sereno \& Rayner, 2003). In this time window, effects of lexical frequency (Hauk \& Pulvermuller, 2004; Hauk et al., 2006a; Sereno et al., 1998; Sereno et al., 2003), syllable frequency (Barber et al., 2004; Hutzler et al., 2004), bi- and trigram frequency (Hauk et al., 2006b) and neighbourhood size (Holcomb et al., 2002) have been observed, indicating that lexical access must have already taken place. The effects of cohort-size reduction observed in the present study occurred slightly later than in these studies, i.e. slightly after 200 ms, even though most of the word has already been processed when the target letter appears. A possible explanation is that, in the current study, the target letter did not complete an actual word: each three-letter string was still only a partial word, congruent with many candidates. The fact that no single candidate is selected, may have caused a delay in the lexical access process. Alternatively, the effects presently observed take place slightly after the initial lexical access stage. That is, although candidates receive activation in an earlier time window, the actual process of inhibiting non-matching candidates does not start until shortly after the initial activation. Future research should address this issue, for example by manipulating reduction and frequency effects simultaneously in order to investigate the time courses of both effects.

It is also important to note that the direction of the cohort-size reduction effect we observed in the P2 time window is reversed in comparison with the effects of syllable frequency and neighbourhood size reported in previous studies (Barber et al., 2004;

92 Holcomb et al., 2002; Hutzler et al., 2004). I.e. we observed more positive amplitudes in conditions where lots of inhibition takes place, whereas previous results showed less positive amplitudes for conditions where many items were activated. In this respect, it can be informative to have a look at the results at letter positions one and two. The results at the first letter presentation replicated previously reported results. That is, P2 amplitudes in response to the presentation of the first letter were less positive for letters with which many words can be formed, which is in line with previous results where high frequency first syllables and stimuli with many orthographic neighbours lead to less positive P2 amplitudes. Comparing results at letter position one with those at position three confirms 
that two effects with opposing directions are taking place in the P2 time window: initial activation of candidates decreases P2 amplitude, whereas inhibition of candidates that do not match input anymore increases it. Extra support for this observation comes from the results at position two. At this position, the condition with the lowest amount of possible candidates was also the condition with the lowest amount of cohort-size reduction compared to position one. In other words: at letter position two, the counteracting effects of candidate activation and inhibition should lead to a null-effect on P2 amplitude. Indeed, no significant difference between conditions could be observed in the P2 amplitude, nor did the effect approach significance. The opposing direction of the effects of activation and inhibition raises the important question what the strength of each of these effects is. It also implies that previously observed P2 effects are actually smaller than they would have been if the inhibition factor would have kept constant. The design of the current study is not suitable for estimating the magnitude of each effect. A design including a condition in which cohort-size reduction is kept constant but cohort activation is parametrically varied would yield valuable additional information.

\section{N400 time window}

The second selection-related effect was observed in the later negative component, at left temporal sites. This negativity scaled with cohort-size reduction in a similar manner as the P2 effect. The distribution of the effect in this time window (left temporal focus) and the duration of the effect (360 - 440 ms after onset of the third letter) imply that the observed component is not a "standard" N400, which is usually characterized as having a centro-parietal distribution slightly biased towards the right hemisphere $300-600 \mathrm{~ms}$ after onset of the critical stimulus (Kutas \& van Petten, 1994). The function of the N400 is related to semantic integration within sentences (Kutas \& Hillyard, 1984) and across sentences and discourse processing (van Berkum et al., 1999). The N400 has also been reported in the auditory domain as being sensitive to both context-based (top-down) and acoustic phonetic (bottom-up) information (van den Brink et al., 2006; van Petten et al., 1999), indicating its involvement in lexical access. There are several reasons why we did not observe a "standard" N400. For example, in our visual gating paradigm, we looked into the integration at a letter-by-letter manner and it could well be that a letter-by-letter integration is reflected in a relatively "smaller" N400. Another reason for the lack of a standard N400 could be that we manipulated neither the amount of active lexical items nor the difficulty of integrating the current input into a context. The amount of lexical candidates was equal between conditions after presentation of the third letter, and letter strings were presented without a semantic context. Furthermore, our participants were never asked to zoom into single lexical items because the cohort size of the three-letter word fragments was always bigger than one. 
Nevertheless, the effect observed in the present study temporally and morphologically overlaps with the early window of the "standard" N400 effect. As reported above, these early stages of the $\mathrm{N} 400$ are likely to be involved in phonological stimulus analysis (van den Brink et al., 2006; van Petten et al., 1999). We suggest that our observed N400like effect reflects integration processes in the mental lexicon. More specifically, given the participants' task to process pseudowords on a letter-by-letter basis, we suggest that the effect reflects the updating of the cohort of active candidates in the mental lexicon. This update involves the adjustment of the cohort from second to third letter input, which entails the inhibition of non-matching items that were activated by the preceding partial input. The relatively late time window in which the update occurs (N400, $360-440 \mathrm{~ms}$ after onset) suggests that it is a consequence of the relatively early inhibitory process (P2, 212-280 ms after third letter onset).

As with the P2 time window, it might be informative to look at effects in the N4 time window at letter positions one and two. In contrast to the P2, effects of cohort-size reduction in the current study are in the same direction as effects of syllable frequency and neighbourhood frequency in previous studies in this time window. Thus we should observe effects of cohort activation and inhibition at all three letter positions. Interestingly, no effects of condition were observed at letter position one. An explanation for this is that no update of the cohort of active candidates is required after presentation of the first letter. If the early N400 time window indeed reflects the update of the activated lexical candidates as more information becomes available, then effects of cohort size should only be expected after an initial cohort of candidates has been activated by previously presented information. This was the case only after presentation of letters two and further, hence the absence of any differences in this time window at letter position one. In line with this interpretation, effects of condition were observed in this time window after presentation of the second letter, indicating that lexical processing is taking place. Observed amplitudes were lowest for the 'low' condition where cohort size and cohort reduction (from position one to two) were smallest, followed by the 'medium' and the 'high' group, respectively. Since the 'medium' group had the highest amount of reduction from position one to two, but the 'high' group had the highest cohort size at position two, this implicates that the

94 effect of cohort size in this time window has a bigger effect on ERP amplitude than does cohort reduction. However, since our study was designed to test for inhibition effects at position three, not activation effects at other positions, better controlled studies should be employed in the future to investigate the strength of both effects.

Stimuli were presented in an unnatural manner during this experiment. With a few exceptions such as letter-by-letter message displays at animated advertisement boards, at car radio fronts, or with text messaging at mobile phones, people do not often encounter written stimuli that are presented to them in an incremental fashion in daily life. Generally, 
words are fixated once before the next word is processed. Although a single fixation is enough to extract the visual information about a written word, fixation times are vulnerable to factors such as word length, frequency and familiarity (Sereno \& Rayner, 2003), and some models of written language comprehension suggest that lexical access in written words takes place in a left-to-right fashion (e.g. Coltheart et al., 2001; Johnson \& Pugh, 1994). In line with this idea, word recognition experiments showed that words with an early Orthograpic Uniqueness Point (OUP, the visual analog of the Uniqueness Point that is used in the auditory gating paradigm) are recognized faster than those with a late OUP (Coltheart \& Rastle, 1994; Kwantes \& Mewhort, 1999; Lindell et al., 2005; Rastle \& Coltheart, 1999). Thus, it seems reasonable to assume that at least in some situations lexical access in response to written words proceeds in a left-to-right manner. Of course we cannot claim that the effects we observed with the current paradigm reflect lexical mechanisms involved in every real-world situation. But by putting time constraints on the availability of orthographic information at the visual input level, as in the current study, rather than within the language processing system, as is generally the case, a time window was created that allowed investigation of the processes involved in cohort-size reduction, and specifically the inhibitory processes associated with this.

In summary, this study has revealed two ERP components related to cohort-size reduction, a factor that has received little attention in the research literature before. We observed a relatively early component (P2) that, we suggest, reflects the neural correlate of the inhibition of candidates based on phonological/orthographic information, followed by a component that mirrors the neural processes involved in updating the active candidates in the lexicon (N400 time window). Given the similarity of the time windows of the present study (cohort-size reduction) and other studies (activation of matching candidates) our data support the plausible assumption that activation of lexical candidates and inhibition of non-matching candidates are closely related in time. In line with others (Barber et al., 2004; Holcomb et al., 2002; Hutzler et al., 2004), our results show that these two phases in the word recognition process can be manipulated separately, and that they independently affect different ERP components.

\section{Acknowledgements}

This project was supported by NWO ASPASIA grant 015.001.053 to B.M. Jansma (Schmitt). The authors would like to thank Boukje Habets, Dirk Köster and Ann-Kathrin Bröckelmann for valuable discussion. 


\section{References}

Allopenna, P. D., Magnuson, J. S., \& Tanenhaus, M. K. (1998). Tracking the time course of spoken word recognition usning eye movements: evidence for continuous mapping models. Journal of Memory and Language 38, 419-439.

Ans, B., Carbonnel, S., \& Valdois, S. (1998). A connectionist multiple-trace memory model for polysyllabic word reading. Psychologocial Review 105, 678-723.

Baayen, R. H., Piepenbrock, R., \& Gulikers, L. (1995). The CELEX Lexical Database (Release 2) [CD-ROM] (Philadelphi, PA, Linguistic Data Consortium, University of Pennsylvania).

Barber, H., Vergara, M., \& Carreiras, M. (2004). Syllable-frequency effects in visual word recognition: evidence from ERPs. Neuroreport 15, 545-548.

Carreiras, M., Vergara, M., \& Barber, H. (2005). Early Event-related Potential Effects of Syllabic Processing during Visual Word Recognition. Journal of Cognitive Neuroscience 17, 1803-1817.

Coltheart, V., Avons, S. E., Masterson, J., \& Laxon, V. J. (1991). The role of assembled phonology in reading comprehension. Memory and Cognition 19, 387-400.

Cutler, A., \& Clifton, C. E. (1999). Comprehending spoken language: A blueprint of the listener. In: C. M. Brown, and P. Hagoort, (Eds.), The neurocognition of language processing. Oxford, Oxford University Press, pp. 123-166.

Coltheart, M., \& Rastle, K. (1994). Serial processing in reading aloud: Evidence for dualroute models of reading. Journal of Experimental Psychology: Human Perception and Performance 20, 1197-1211.

Coltheart, M., Rastle, K., Perry, C., Langdon, R., \& Ziegler, J. (2001). DRC: a dual route cascaded model of visual word recognition and reading aloud. Psychol Rev 108, 204-256.

Dahan, D., Magnuson, J. S., \& Tanenhaus, M. K. (2001). Time course of frequency effects in spoken-word recognition: evidence from eye movements. Cognitive Psychology 42, 317-367.

Dahan, D., \& Tanenhaus, M. K. (2004). Continuous mapping from sound to meaning in spoken-language comprehension: immediate effects of verb-based thematic constraints. Journal of Experimental Psychology: Learning, Memory, and Cognition 30, 498-513.

Friedrich, C. K., Kotz, S. A., Friederici, A. D., \& Gunter, T. C. (2004). ERPs reflect lexical identification in word fragment priming. J Cogn Neurosci 16, 541-552.

Friedrich, C. K. (2005). Neurophysiological correlates of mismatch in lexical access. BMC Neurosci 6, 64. 
Gaskell, M. G., \& Marslen-Wilson, W. D. (1997). Integrating form and meaning: A distributed model of speech perception. Language and Cognitive Processes 12, 613-656.

Goldinger, S. D., Luce, P. A., Pisoni, D. B., \& Marcario, J. K. (1992). Form-based priming in spoken word recognition: the roles of competition and bias. Journal of Experimental Psychology: Learning, Memory, and Cognition 18, 121 1-1238.

Greenhouse, S., \& Geisser, S. (1959). On methods in the analysis of profile data. Psychometrika 24, 95-112.

Guillem, F., Bicu, M., \& Debruille, J. B. (2001). Dissociating memory processes involved in direct and indirect tests with ERPs to unfamiliar faces. Cognitive Brain Research 11, 113-125.

Hagen, G. F., Gatherwright, J. R., Lopez, B. A., \& Polich, J. (2006). P3a from visual stimuli: task difficulty effects. International Journal of Psychophysiology 59, 8-14.

Handy, T. C., \& Mangun, G. R. (2000). Attention and spatial selection: electrophysiological evidence for modulation by perceptual load. Perception Psychophysiology 62, 175-186.

Hauk, O., \& Pulvermuller, F. (2004). Effects of word length and frequency on the human event-related potential. Clin Neurophysiol 115, 1090-1 103.

Hauk, O., Davis, M. H., Ford, M., Pulvermuller, F., \& Marslen-Wilson, W. D. (2006a). The time course of visual word recognition as revealed by linear regression analysis of ERP data. Neuroimage 30, 1383-1400.

Hauk, O., Patterson, K., Woollams, A., Watling, L., Pulvermuller, F., \& Rogers, T. T. (2006b). [Q:] When would you prefer a SOSSAGE to a SAUSAGE? [A:] At about $100 \mathrm{msec}$. ERP correlates of orthographic typicality and lexicality in written word recognition. J Cogn Neurosci 18, 818-832.

Holcomb, P. J., Grainger, J., \& O'Rourke, T. (2002). An electrophysiological study of the effects of orthographic neighborhood size on printed word perception. Journal of Cognitive Neuroscience 14, 938-950.

Hutzler, F., Bergmann, J., Conrad, M., Kronbichler, M., Stenneken, P., \& Jacobs, A. M. (2004). Inhibitory effects of first syllable-frequency in lexical decision: an eventrelated potential study. Neuroscience Letters 372, 179-184.

Jasper, H. H. (1958). The ten-twenty electrode system of the international federation in electroencephalography and clinical neurophysiology. EEG Journal 10, 371375.

Johnson, N.F., \& Pugh, K.R. (1994). A cohort model of visual word recognition. Cognitive Psychology 26, 240-346. 
Kenemans, J. L., Kok, A., \& Smulders, F. T. (1993). Event-related potentials to conjunctions of spatial frequency and orientation as a function of stimulus parameters and response requirements. Electroencephalography and Clinical Neurophysiology $88,51-63$.

Kutas, M., \& Hillyard, S. A. (1980). Reading senseless sentences: brain potentials reflect semantic incongruity. Science 207, 203-205.

Kutas, M., \& Hillyard, S. A. (1984). Brain potentials during reading reflect word expectancy and semantic association. Nature 307, 161-163.

Kutas, M., \& van Petten, C. (1994). Psycholinguistics electrified. Event-related brain potential investigations. In: M. A. Gernsbacher, (Eds.), Handbook of psycholinguistics. San Diego, CA, Academic Press.

Kwantes, P. J., \& Mewhort, D. J. K. (1999). Evidence for sequential processing in visual word recognition. Journal of Experimental Psychology: Human Perception and Performance 25, 376-381.

Lindell, A. K., Nicholls, M. E., Kwantes, P. J., \& Castles, A. (2005). Sequential processing in hemispheric word recognition: the impact of initial letter discriminability on the OUP naming effect. Brain Lang 93, 160-172.

Marslen-Wilson, W. D. (1987). Functional parallelism in spoken word-recognition. Cognition 25, 71-102.

Masson, M. E. J. (1995). A distributed memory model of semantic priming. Journal of Experimental Psychology: Learning, Memory, and Cognition 21, 3-23.

McClelland, J. L., \& Elman, J. L. (1986). The TRACE model of speech perception. Cognitive Psychology 18, 1-86.

Melara, R. D., Rao, A., \& Tong, Y. (2002). The duality of selection: excitatory and inhibitory processes in auditory selective attention. Journal of Experimental Psychology: Human Perception and Performance 28, 279-306.

Norris, D., McQueen, J. M., \& Cutler, A. (1995). Competition and segmentation in spoken-word recognition. Journal of Experimental Psychology: Learning, Memory, and Cognition 21, 1209-1228.

Perfetti, C. (1999). Comprehending written language: A blueprint of the reader. In: C. M. Brown, and P. Hagoort, (Eds.), The neurocognition of language processing. Oxford, Oxford University Press, pp. 167-208.

Potts, G. F., Dien, J., Hartry-Speiser, A. L., McDougal, L. M., \& Tucker, D. M. (1998). Dense sensor array topography of the event-related potential to task-relevant auditory stimuli. Electroencephalography and Clinical Neurophysiology 106, 444-456.

Potts, G. F. (2004). An ERP index of task relevance evaluation of visual stimuli. Brain and Cognition 56, 5-13. 
Pylkkanen, L., Stringfellow, A., \& Marantz, A. (2002). Neuromagnetic evidence for the timing of lexical activation: an MEG component sensitive to phonotactic probability but not to neighborhood density. Brain Lang 81, 666-678.

Pylkkanen, L., Feintuch, S., Hopkins, E., \& Marantz, A. (2004). Neural correlates of the effects of morphological family frequency and family size: an MEG study. Cognition 91, B35-45.

Pylkkanen, L., Llinas, R., \& Murphy, G. L. (2006). The representation of polysemy: MEG evidence. J Cogn Neurosci 18, 97-109.

Sereno, S. C., Rayner, K., \& Posner, M. I. (1998). Establishing a time-line of word recognition: evidence from eye movements and event-related potentials. Neuroreport 9, 2195-2200.

Rastle, K., \& Coltheart, M. (1999). Serial and strategic effects in reading aloud. Journal of Experimental Psychology: Human Perception and Performance 25, 482-503.

Sereno, S. C., Brewer, C. C., \& O'Donnell, P. J. (2003). Context effects in word recognition: evidence for early interactive processing. Psychol Sci 14, 328-333.

Sereno, S. C., \& Rayner, K. (2003). Measuring word recognition in reading: eye movements and event-related potentials. Trends Cogn Sci 7, 489-493.

Smid, H. G., Jakob, A., \& Heinze, H. J. (1999). An event-related brain potential study of visual selective attention to conjunctions of color and shape. Psychophysiology 36, 264-279.

Spivey, M. J., Grosiean, M., \& Knoblich, G. (2005). Continuous attraction toward phonological competitors. Proceedings of the National Academy of Sciences of the United States of America 102, 10393-10398.

Stockall, L., Stringfellow, A., \& Marantz, A. (2004). The precise time course of lexical activation: MEG measurements of the effects of frequency, probability, and density in lexical decision. Brain Lang 90, 88-94.

Swaab, T. Y., Camblin, C. C., \& Gordon, P. C. (2004). Electrophysiological evidence for reversed lexical repetition effects in language processing. Journal of Cognitive Neuroscience 16, 715-726.

van Berkum, J. J., Hagoort, P., \& Brown, C. M. (1999). Semantic integration in sentences and discourse: evidence from the N400. Journal of Cognitive Neuroscience 11, 657-671.

van den Brink, D., Brown, C. M., \& Hagoort, P. (2006). The cascaded nature of lexical selection and integration in auditory sentence processing. J Exp Psychol Learn Mem Cogn 32, 364-372. 
van Petten, C., Coulson, S., Rubin, S., Plante, E., \& Parks, M. (1999). Time course of word identification and semantic integration in spoken language. Journal of Experimental Psychology: Learning, Memory, and Cognition 25, 394-417.

van Petten, C., \& Luka, B. J. (2006). Neural localization of semantic context effects in electromagnetic and hemodynamic studies. Brain and Language 97, 279-293.

Zwitserlood, P. (1989). The locus of the effects of sentential-semantic context in spokenword processing. Cognition 32, 25-64. 
○

O

0

$\bigcirc$

U

o 
General discussion 
The goal of the research line described in this thesis was the investigation of visual and linguistic selection mechanisms in the human brain. Attention research has traditionally focused on enhanced processing at attended stimulus locations in light of location-based and feature based attention. The fate of unattended pictures in a display only recently has become the target of investigations. These well-visible but unattended stimuli usually do not trigger an explicit response, but are able to influence our behaviour. As neuroimaging and electrophysiological methods can measure neural activity associated with unattended stimuli both in the presence and the absence of an overt response, they were the method of choice for the research described in this thesis. The research described here explored whether 'biased competition', an attention mechanism that is well-investigated in monkey neurophysiology, is also a primary selection mechanism in human visual cortex. Besides information about the nature of the selection process at a visual level, these types of designs can be used to investigate whether features associated with unattended stimuli survive beyond the visual cortex. Specifically, we investigated whether phonological features of ignored stimuli are identified by phonological processing areas. Finally, a new design was introduced that allows the investigation of online selection of word candidates as more information about their phonology becomes available.

\section{Biased competition in human visual cortex}

A theory that has received considerable support from monkey neurophysiological studies over the last few decades is the 'biased competition' framework. Although it is referred to more and more often in light of human neuroimaging studies, direct tests of the hypotheses generated by biased competition are scarce in human studies. Kastner et al. (Beck \& Kastner, 2005; Kastner et al., 1998; 1999; 2001a; Pinsk et al., 1999), have shown that simultaneously presented stimuli compete for neural resources in human visual cortex; they observed a lower amount of BOLD-signal change when stimuli were simultaneously presented (i.e. competed for neural resources) compared to sequential presentations (i.e. not competing for neural resources). A potential problem with this account is the comparison between simultaneous and sequential stimuli: since a higher

104 amount of transient stimulus onsets (as is the case in sequential presentation) can lead to higher activations (Fox \& Raichle, 1985; Rees et al., 1997), the observed difference between simultaneous and sequential conditions might be explained by the different presentation rate of the stimuli. Therefore, a stronger case for the biased competition approach would be made if a design is used that eliminates the use for a sequential condition. In chapter 2 we introduced such a design, in which stimuli were always presented simultaneously. By looking at the difference between attended and unattended stimulus displays as a measure of the amount of competition, we corroborated and extended earlier findings 
and showed that competition in human visual cortex depends on the spatial separation between stimuli and the RF size of a given area, as is predicted by the biased competition framework.

Further support that biased competition acts as a dominant selection mechanism in human visual cortex was presented in chapter 3 . The design introduced in chapter 2 was applied in an ERP setting, in which the basic results of an increased attention effect with smaller spatial separations was replicated. In addition, due to the high temporal resolution of ERP, it enabled us to investigated the time course of the suppressive and biasing mechanisms. We observed that in early visual components, only effects of spatial separation were present. In line with a biased competition account, these effects showed smaller ERP amplitudes for smaller display sizes, indicating higher amounts of competition. Attention effects were observed at the N1 and later ERP components but, interestingly, occurred earlier at anterior electrode sites than at posterior sites, indicating a mediating effect of attention effects in visual areas by higher-order areas.

An issue that needs to be addressed, is that the original neurophysiological studies showed competition in single cells between preferred and non-preferred stimuli. Such studies showed that the firing rate of a neuron is a weighted average of the firing rates of the single stimuli (e.g. Chelazzi et al., 2001; Moran \& Desimone, 1985). The approach hence relies on observations in the receptive field of a single neuron. cell. In FMRI, a voxel (the smallest unit in an fMRI dataset) will contain many cells with different tuning curves for the features they respond to. With the complex, colourful pictures used in the present paradigm, we did not distinguish between preferred and non-preferred stimuli within voxels or areas. In fact, a single voxel should contain cells that prefer stimulus $A$ and not $B$, and vice versa. Therefore, within a voxel, one could expect the weighted average of the responses to both stimuli to be the same as the response to one of the single stimuli. In that case, no difference in average competition in a group of cells would be observed. That, in turn, could suggest that the differences between high- and low-competition conditions that were observed in the previous fMRI studies might not reflect differences in competition in unattended conditions, but rather a facilitative effect of attention in the attended conditions. However, although such an alternative explanation would also predict increased activation in attended conditions compared to unattended ones, it cannot explain the dependence of attention effects on the spatial separation between stimuli and the RF size of an area (Kastner et al. 1998; 2001a; chapter 2). It also cannot explain the previously reported competitive effects that were observed in the absence of attention (Beck \& Kastner, 2005; Kastner et al., 1998; 1999; 2001 a; Pinsk et al., 1999).

A recent study by Kamitani and Tong (2005) may provide the basis for new experiments that can shed light on this discussion. In an fMRI study, they showed that early visual areas have a response bias towards gratings of a certain orientation that differs from voxel to voxel. This means that cells who optimally respond to that orientation must be slightly 
overrepresented in certain voxels. Consequently, it is possible to define preferred and non-preferred stimuli per voxel, and measure how they respond to the presentation of both of these in the presence and absence of visual attention. Kamitani and Tong already showed that when two gratings with differing orientation are presented simultaneously, the activity within a given voxel is biased towards the attended orientation. However, a critical future test for the biased competition approach would also involve measuring each voxel's response when visual attention is directed away from these stimuli.

An alternative way to explore competitive effects between effective and non-effective stimuli is to make use of the stimulus selectivity of certain visual areas like the Fusiform Face Area (FFA, responsive to faces (Kanwisher et al., 1997)), the Lateral Occipital Complex (LOC, responsive to objects (Grill-Spector et al., 1998)) or the Parahippocampal Place Area (PPA, responsive to scenes (Epstein \& Kanwisher, 1998)). This means that it is possible to define preferred and non-preferred stimuli for FFA, and measure its response when these are simultaneously presented (Gentile et al., 2007; Jacques \& Rossion, 2004; O'Craven \& Kanwisher, 2000; Yi et al., 2004).

It is important to note that although stimulus selectivity has been repeatedly demonstrated for areas such as the FFA and PPA, some authors have proposed that the time courses of voxels in these regions carry enough information to distinguish not only activations associated with stimuli the region is most selective for (like faces or houses), but also a variety of stimulus categories such as chairs, shoes, cows, and even greebles (Cox \& Savoy, 2003; Haxby et al., 2001). This information can be utilised by means of multivariate analysis methods, which detect activation patterns across a range of voxels. With powerful analysis tools like these, it becomes possible to investigate whether the activation level in a group of voxels represents either one of the presented stimuli, or a mixture of both.

\section{Fate of ignored stimuli}

An issue that has occupied researchers for decades is whether ignored stimuli are filtered out completely by attentional mechanisms, or whether they are represented in

106 attenuated form in areas beyond the visual cortex. Although many behavioural studies have addressed this issue (see chapter 4), results are inconclusive at best, with ample evidence for both early and late selection views. Neuroimaging studies designed to investigate the fate of ignored stimuli, on the other hand, have thus far mainly focused on visual areas (Bentley et al., 2003; Eger et al., 2004; Murray \& Wojciulik, 2004; Rees et al., 1999; Yi et al., 2004). The results presented in chapter 4 are amongst the first to show that, under some circumstances, the neural correlates of phonological properties 
of ignored stimuli can be detected in phonological processing areas. This means that the names of pictures that are outside the focus of our attention can be internally processed when resource availability allows for it.

When investigating effects of ignored stimuli, it is vital to distinguish between stimuli that are actively being ignored (e.g. because they occur at non-target locations) and stimuli that fail to reach awareness. In the literature, Rapid Visual Stimulation Paradigms (RVSP) and masked priming are often used to investigate whether "ignored" stimuli are identified. In these paradigms, visual stimulation occurs at such a high rate that not all stimuli can be reported by participants (see Keysers \& Perrett (2002)). However, in these paradigms stimuli are not actively ignored, as is the case in our paradigms, but fail to reach awareness because the stimulus representation is overwritten by a new stimulus, or the executive system is occupied with the processing of a previous target. In this respect RSVP and priming paradigms are more suited to investigate temporal limitations of our cognitive systems than spatial ones, which should be investigated by the simultaneous presentation of multiple stimuli.

From the results presented in chapter 2, it is apparent that it is important to take into account the spatial location of simultaneously presented stimuli. Since competition for neural resources arises at least in part from the limited processing capacity within RFs, different results can be expected when stimuli are presented at the same (e.g. O'Craven \& Kanwisher, 2000; Yi et al., 2004) or spatially separable locations (Bentley et al., 2003; Eger et al., 2004, and the designs used in this thesis). When overlapping stimuli are used, the same stimuli necessarily occupy the same RFs in almost every neuron involved in their processing. This will maximise the competition for neural resources and, in case the target is attended, minimise processing of the distractor stimulus (Lavie, 1995). A different situation arises, however, when stimuli are presented at non-overlapping locations. Although these stimuli will compete for resources in some neurons (depending on RF size and stimulus separation, see chapters 2 and 3), there will also be neurons, especially in lower order areas whose RF will contain only one stimulus. These neurons will not experience competitive effects, and ignored stimuli will be represented by a subset of neurons within these visual areas. Via this mechanism, it is conceivable that at least physical properties of these ignored stimuli are extracted in early visual areas. Due to the extensive interconnectedness between cortical areas (van Essen et al., 1984), these representations could be sent to higher-order areas where, in some cases, feature identification takes place, such as the identification of phonological properties (see chapter 4).

We observed that when participants were performing an offset monitoring task, BOLD-signal change in Wernicke's area and the inferior frontal gyrus (IFG) was higher for unrelated than for related conditions (see chapter 4). It is difficult to interpret these results in terms of linguistic selection mechanisms due to the interaction with processes at a visual level and higher-order executive processes. Whereas single-cell recordings in monkeys 
have revealed that, in visual cortex, neural firing rates in response to multiple stimuli reflect the average firing rate of the single stimuli, it is not clear what to expect in terms of competitive effects in linguistic areas. Extrapolating from the results in the visual cortex, it seems plausible to assume that for a given neuron in, say, Wernicke's area or the IFG, firing rates are high for certain phonological features (e.g. plosive sounds, certain frequency bands or formant combinations), but low for others. Let us term the range of phonological properties this neuron responds to (either in an excitatory of an inhibitory fashion) the RF of that neuron. The question then is what happens if competing phonological properties are presented to this neuron, as is the case in unrelated trials in picture-word or picture-picture interference paradigms, or the paradigm used in chapter 4 . One possibility is that, similar to visual stimuli, competing phonological properties inhibit each other's representation, leading to a suppressed activation in these areas compared to related trials. Alternatively, these phonological properties could activate separate populations of neurons, leading to an increased activation for unrelated conditions, compared to related ones.

In general, higher hemodynamic responses for unrelated trials (de Zubicaray et al., 2002) or slower behavioural responses for unrelated trials compared to related ones (Jescheniak \& Schriefers, 2001; Morsella \& Miozzo, 2002; Navarrete \& Costa, 2005; Starreveld \& La Heii, 1996) have been interpreted as a facilitative effect in related trials, compared to unrelated ones. The increased activation for unrelated compared to related blocks in Wernicke's area and the IFG in the offset monitoring task indicate the recruitment of different populations of neurons involved in phonological encoding. The competition of these populations may lead to slower processing times, and hence the slower reaction times often observed in behavioural experiments. However, the spatial resolution of fMRI is not high enough to distinguish activation in different subpopulations of these areas.

Nevertheless, it is important to note that behavioural responses to pictures with a phonologically related distractor is actually slower than those to a control picture where the distractor stimulus consists of a row of X's (Starreveld \& La Heii, 1995; 1996). Thus, although phonologically related distractors cause less interference than unrelated ones, they still slow down responses compared to control stimuli. Hence, more processing resources may be required in unrelated conditions, and an increase in BOLD-signal

108 change in areas involved in phonological processing in unrelated conditions could be caused by stronger top-down influences of executive areas (de Zubicaray et al, 2002). The current design is not suited for investigating the direction of influences from one brain area to another. Future studies of selection mechanisms in higher-order domains could make use of methods like Granger causality mapping (Roebroeck et al., 2005) or structural equation modeling (Mclntosh \& Gonzalez-Lima, 1994) to investigate directed influences of certain cortical areas to others.

Although we do not know the exact mechanisms involved in the interactions between stimuli in phonological processing areas, the results strongly imply that phonological 
features of unattended distractor pictures can be identified under some circumstances. This yields important information about the functioning of visual selection mechanisms, since it means that representations of distractor stimuli are not completely filtered out at a visual level, but rather are attenuated. In addition, the paradigm introduces a starting point from which selection mechanisms in linguistic areas can be investigated.

\section{Selection in the absence of visual selection}

As emphasised in the previous section, it is important to take into account the processes occurring at a visual level in order to interpret the higher-order processing of the language features of multiple stimuli. That is, stimuli that are biased in visual cortex, are likely to gain a processing advantage also in higher-order areas, which may reduce the chances of picking up distractor-related processes. The design introduced in chapter 5 basically served to investigate selection processes in language modules in the absence of differential processing at a visual level. This paradigm, that is based on the auditory gating paradigm often used in language comprehension studies (van Petten et al., 1999; Zwitserlood, 1989), manipulates the amount of activated lexical candidates without manipulating visual features. By spelling (parts of) words out on the screen in a letter-byletter fashion, a time window is created in which we can measure the activity associated with the updating of the cohort of lexical candidates that still matches the current input. Although stimuli were presented in a somewhat unnatural manner (i.e. besides electronic billboards and some text messaging, we hardly ever see words spelled out letter-by-letter), we observed ERP effects that parametrically scaled with the amount of candidates that needed to be inhibited after the presentation of a new letter on the screen. These findings indicate that this paradigm is suitable for investigating effects of activation and especially inhibition of lexical candidates as more information becomes available. Importantly, although the letters on the screen never formed a real word, it would be interesting to see if neural correlates of the final selection can be observed when only one candidate is left, or whether only effects of cohort reduction will be observed. An interesting expansion of the possibilities with this paradigm lies in new analysis methods like a linear regression analysis of ERP data (Hauk et al., 2006). With this method, the amplitude or latency of ERP components in single trials is the dependent variable in a linear regression model with, for example, factors such as lexical frequency, word length, neighbourhood size, or cohort-size reduction as regressors. The advantage of this method is that it is no longer necessary to bin stimuli into arbitrary stimulus categories (e.g. high vs. low frequency), but that the stimuli can represent a continuous sample from the whole range of possible values for a certain feature.

An interesting application of the paradigm introduced in chapter 5 could be to not only base cohort-size reduction on orthographic or phonological information, but also 
on semantic properties. For example, as a sentence unfolds, the subject of the sentence should become clearer, reducing the amount of possible candidates. Many studies have addressed ERP responses in reaction to words that match the anticipated candidate and those that do not, the latter usually eliciting larger N400 components than the former (Kutas \& Hillyard, 1984; van Berkum et al., 1999). With a modified version of the paradigm presented in chapter 5, however, one could also investigate the brain's responses to words that restrict the amount of candidates to a large degree compared to those that lead to small restrictions.

To summarise, in this thesis I have presented support for the view that stimuli compete for neural representation in human visual cortex (chapters 2 and 3), as predicted by a biased competition account. Importantly, although higher-order areas can bias this competitive process through attention, our results imply that, at least under some circumstances, attention merely attenuates the neural representation of unattended distractor pictures, rather than filter them out completely. This is evident from the observed phonological processing of these pictures (chapter 4), which could not have taken place if the features of the unattended picture were filtered out completely. Selection also takes place in higher-order cortical areas involved in, for example, phonological language processing, but the processing of simultaneously presented stimuli by these areas is necessarily influenced by competitive processes at the perceptual level. This makes the interpretation of selection mechanisms in these areas more difficult, and more work is needed to identify the exact mechanism at work here. To be able to investigate questions of selection based on phonological properties, I introduced a design that allows for the manipulation of selection requirements in a highly controlled manner, without the side effects of stimulus competition at a visual level (chapter 5). The work presented in this thesis, with its combination of paradigms and research methods, contributes to our understanding of visual and phonological selection mechanisms, but at the same time reflects that our understanding of these mechanisms is still fairly limited, especially in higher-order cognitive tasks, such as language processing. 


\section{References}

Beck, D. M., \& Kastner, S. (2005). Stimulus context modulates competition in human extrastriate cortex. Nat Neurosci 8, $1110-1116$.

Bentley, P., Vuilleumier, P., Thiel, C. M., Driver, J., \& Dolan, R. J. (2003). Effects of attention and emotion on repetition priming and their modulation by cholinergic enhancement. J Neurophysiol 90, 1171-1181.

Chelazzi, L., Miller, E. K., Duncan, J., \& Desimone, R. (2001). Responses of Neurons in Macaque Area V4 During Memory-guided visual Search. Cerebral Cortex 11, $761-772$.

Cox, D. D., \& Savoy, R. L. (2003). Functional magnetic resonance imaging (fMRI) "brain reading": detecting and classifying distributed patterns of $f M R I$ activity in human visual cortex. Neuroimage 19, $261-270$.

de Zubicaray, G. I., McMahon, K. L., Eastburn, M. M., \& Wilson, S. J. (2002). Orthographic/ phonological facilitation of naming responses in the picture-word task: an eventrelated fMRI study using overt vocal responding. Neuroimage 16, 1084-1093.

Eger, E., Henson, R. N., Driver, J., \& Dolan, R. J. (2004). BOLD repetition decreases in object-responsive ventral visual areas depend on spatial attention. J Neurophysiol 92, 1241-1247.

Epstein, R., \& Kanwisher, N. (1998). A cortical representation of the local visual environment. Nature 392, 598-601.

Fox, P. T., \& Raichle, M. E. (1985). Stimulus rate determines regional brain blood flow in striate cortex. Ann Neurol 17, 303-305.

Gentile, F., Bles, M., De Weerd, P., Goebel, R., \& Jansma, B. M. (2007). Neural competition in the visual stream during processing of similar vs. dissimilar faces. Paper presented at: Human Brain Mapping, 13th annual meeting (Chicago).

Grill-Spector, K., Kushnir, T., Edelman, S., Itzchak, Y., \& Malach, R. (1998). Cue-invariant activation in object-related areas of the human occipital lobe. Neuron 21, 191 202.

Hauk, O., Davis, M. H., Ford, M., Pulvermuller, F., \& Marslen-Wilson, W. D. (2006). The time course of visual word recognition as revealed by linear regression analysis of ERP data. Neuroimage 30, 1383-1400.

Haxby, J. V., Gobbini, M. I., Furey, M. L., Ishai, A., Schouten, J. L., \& Pietrini, P. (2001). Distributed and overlapping representations of faces and objects in ventral temporal cortex. Science 293, 2425-2430.

Jacques, C., \& Rossion, B. (2004). Concurrent processing reveals competition between visual representations of faces. Neuroreport 15, 2417-2421. 
Jescheniak, J. D., \& Schriefers, H. (2001). Priming effects from phonologically related distractors in picture-word interference. Q J Exp Psychol A 54, 371 -382.

Kamitani, Y., \& Tong, F. (2005). Decoding the visual and subjective contents of the human brain. Nat Neurosci 8, 679-685.

Kanwisher, N., McDermott, J., \& Chun, M. M. (1997). The fusiform face area: a module in human extrastriate cortex specialized for face perception. J Neurosci 17, $4302-$ 4311.

Kastner, S., De Weerd, P., Desimone, R., \& Ungerleider, L. (1998). Mechanisms of Directed Attention in the Human Extrastriate Cortex as Revealed by Functional MRI. Science 282, 108-111.

Kastner, S., Pinsk, M. A., De Weerd, P., Desimone, R., \& Ungerleider, L. (1999). Increased Activity in Human Visual Cortex during Directed Attention in the Absence of Visual Stimulation. Neuron 22, 751-761.

Kastner, S., De Weerd, P., Pinsk, M. A., Elizondo, M. I., Desimone, R., \& Ungerleider, L. (2001a). Modulation of Sensory Suppression: Implications for Receptive Field Sizes in the Human Visual Cortex. Journal of Neurophysiology 86, 1398-1411.

Keysers, C., \& Perrett, D. I. (2002). Visual masking and RSVP reveal neural competition. Trends Cogn Sci 6, 120-125.

Kutas, M., \& Hillyard, S. A. (1984). Brain potentials during reading reflect word expectancy and semantic association. Nature 307, 161-163.

Lavie, N. (1995). Perceptual load as a necessary condition for selective attention. J Exp Psychol Hum Percept Perform 21, 451-468.

Mclntosh, A. R., \& Gonzalez-Lima, F. (1994). Structural equation modeling and its application to network analysis in functional brain imaging. Human Brain Mapping 2, 2-22.

Moran, J., \& Desimone, R. (1985). Selective attention gates visual processing in the extrastriate cortex. Science 229, 782-784.

Morsella, E., \& Miozzo, M. (2002). Evidence for a cascade model of lexical access in speech production. J Exp Psychol Learn Mem Cogn 28, 555-563.

Murray, S. O., \& Wojciulik, E. (2004). Attention increases neural selectivity in the human lateral occipital complex. Nat Neurosci 7, 70-74.

Navarrete, E., \& Costa, A. (2005). Phonological activation of ignored pictures: Further evidence for a cascade model of lexical access. Journal of Memory and Language 53, 359-377.

$\mathrm{O}^{\prime}$ Craven, K. M., \& Kanwisher, N. (2000). Mental imagery of faces and places activates corresponding stiimulus-specific brain regions. J Cogn Neurosci 12, 1013 1023. 
Pinsk, M. A., Kastner, S., Desimone, R., \& Ungerleider, L. (1999). An estimate of receptive field sizes in human visual cortex. Neurolmage 9, s885.

Rees, G., Howseman, A., Josephs, O., Frith, C. D., Friston, K. J., Frackowiak, R. S., \& Turner, R. (1997). Characterizing the relationship between BOLD contrast and regional cerebral blood flow measurements by varying the stimulus presentation rate. Neuroimage 6, 270-278.

Rees, G., Russell, C., Frith, C. D., \& Driver, J. (1999). Inattentional blindness versus inattentional amnesia for fixated but ignored words. Science 286, 2504-2507.

Roebroeck, A., Formisano, E., \& Goebel, R. (2005). Mapping directed influence over the brain using Granger causality and fMRI. Neuroimage 25, 230-242.

Starreveld, P. A., \& La Heii, W. (1995). Semantic interference, orthographic facilitation, and their interaction in naming tasks. Journal of Experimental Psychology: Learning, Memory, and Cognition 21, 686-698.

Starreveld, P. A., \& La Heii, W. (1996). Time-course analysis of semantic and orthographiccontext effects in picture naming. Journal of Experimental Psychology: Learning, Memory, and Cognition 22, 896-918.

van Berkum, J. J., Hagoort, P., \& Brown, C. M. (1999). Semantic integration in sentences and discourse: evidence from the N400. Journal of Cognitive Neuroscience 11, 657-671.

van Essen, D. C., Newsome, W. T., \& Maunsell, J. H. (1984). The visual field representation in striate cortex of the macaque monkey: asymmetries, anisotropies, and individual variability. Vision Res 24, 429-448.

van Petten, C., Coulson, S., Rubin, S., Plante, E., \& Parks, M. (1999). Time course of word identification and semantic integration in spoken language. Journal of Experimental Psychology: Learning, Memory, and Cognition 25, 394-417.

Yi, D. J., Woodman, G. F., Widders, D., Marois, R., \& Chun, M. M. (2004). Neural fate of ignored stimuli: dissociable effects of perceptual and working memory load. Nat Neurosci 7, 992-996.

Zwitserlood, P. (1989). The locus of the effects of sentential-semantic context in spokenword processing. Cognition 32, 25-64. 

Summary 
The work described in this thesis investigated the nature of competitive and selection mechanisms in the human brain. The framework in which this investigation took place, is the 'biased competition' theory, which has received a considerable amount of attention in monkey neurophysiology, and has recently begun to be explored in human visual cortex. The core assumption of this theory is simultaneously presented stimuli compete for neural resources. Since the processing capacity of cortical areas and single neurons is limited, not every stimulus can be represented to the full extent, and they suppress each other's representation. Importantly, this competition can be biased in favour of a stimulus through bottom-up mechanisms like saliency, or through top-down mechanisms such as focused attention. The processing of this stimulus will take precedence over that of others, and the neural activation pattern will represent its presence, and not that of the distractors.

Support for hypotheses derived from a biased competition approach is reported in chapter 2. Specifically, since competition for neural resources arises when multiple stimuli are presented within a receptive field (RF) of a neuron, it should decrease when stimuli are placed further apart so that less stimuli are present within an RF. In addition, due to the increase in RF size higher up in the visual processing hierarchy, stimuli should fall within an RF more often in higher-order visual areas like V4 and TEO. In line with these hypotheses, we observed with functional Magnetic Resonance Imaging (fMRI) that the amount of competition between stimuli in human visual cortex decreased when stimuli were spaced further apart. In addition, increased competitive interactions were observed in higher-order visual areas. These results provide further support that biased competition acts as a dominant selection mechanism in the human visual cortex.

Whereas the results obtained with $\mathrm{fMRI}$ in chapter 2 provide a high spatial resolution to locate effects of competitive interactions in visual areas, the high temporal resolution of event-related potentials (ERPs) was employed in the study described in chapter 3 to investigate the time course of these interactions. By measuring the electrical voltage fluctuations at the scalp in response to simultaneously presented stimuli, we observed that there is an early time window (from approximately $100-150 \mathrm{~ms}$ ) after stimulus onset in which stimuli compete for neural resources regardless of the location of attention. Only after an initial analysis of the stimuli by higher-order areas does attention come into play

116 to bias the competition between stimuli in favour of the attended item. These results are the first that highlight the temporal aspects of competitive interactions in human visual cortex.

The fact that stimuli compete for neural representations evokes the question what exactly happens to ignored distractor stimuli. In chapter 4, a study is described that investigated whether cortical areas involved in phonological processing, like Wernicke's area, the inferior frontal gyrus, and the insula, have representations of phonological features of distractor stimuli. In particular, we presented participants with two pictures whose names were either phonologically related (e.g. 'fox' - 'fork'), or unrelated (e.g. 'fox' 
- 'rake'). Participants attended either one of these pictures, or performed a control task in which both pictures were ignored. The rationale behind this experiment was that if the relatedness between these pictures is detected by the brain, the phonological properties of both pictures must have been identified. In line with this idea, we observed that under some task conditions the brain responded differently to related and unrelated picture pairs. This indicates that these pictures are identified and their name activated, even if the pictures are ignored.

Although the results of chapter 4 revealed that phonological properties of ignored stimuli can be identified by the brain, the design employed in this study could not be used to measure the strength and direction of competitive interactions in regions beyond the visual cortex. Hence, a design was needed to study selection process in linguistic areas in the absence of visual competition between stimuli. Such a design is described in chapter 5, where words were spelled out on the screen letter by letter, and in which we manipulated the amount of words that were compatible with the presented letters. The benefit of this design was that it was possible to manipulate the amount of words that need to be inhibited when a new letter is presented, without introducing attentional biases or visual stimulus differences, as was the case in chapter 4 . We observed two ERP components that scaled with the amount of words that needed to be inhibited, showing for the first time that processes of lexical selection can be observed in the ERP that depend on the amount of inhibited candidates, rather than the amount of activated ones.

In summary, the studies described in this thesis investigated the role of selection mechanisms in the human brain, both in areas involved in visual processing (chapters 2 and 3), and in linguistic areas (chapters 4 and 5). By using methods both with a high spatial resolution, like fMRI (chapters 2 and 4), and those with a high temporal resolution, like ERP (chapters 3 and 5), a constructive picture is emerging about the nature of the selection mechanisms in the human brain. This, as well as practical considerations, future directions and general remarks are discussed in chapter 6. 

Samenvatting 
Het werk in dit proefschrift richt zich op het karakter van competitieve en selectiemechanismen in het menselijke brein. Het onderzoek is gebaseerd op de 'biased competition' theorie, die veel aandacht heeft gekregen in neurophysiologische studies met primaten, en die recent ook toegepast is op de menselijke visuele cortex. The basisaanname van deze theorie is dat gelijktijdig gepresenteerde stimuli strijden om neurale hulpbronnen. Aangezien corticale gebieden en neuronen een beperkte verwerkingscapaciteit hebben, kan niet iedere stimulus volledig gerepresenteerd worden, wat leidt tot een onderdrukking van elkaars representatie. Deze competitie kan beslecht worden in het voordeel van één van de stimuli door middel van bottom-up mechanismen als salientie, of door top-down mechanismen als gerichte aandacht. De verwerking van deze stimulus zal bevoordeeld worden ten opzichte van die van anderen, en het neurale activatiepatroon zal deze stimulus representeren, en niet dat van distractoren.

Ondersteuning voor hypotheses gebaseerd op de "biased competition" theorie worden gerapporteerd in hoofdstuk 2. Omdat competitie voor neurale hulpbronnen optreedt wanneer meerdere stimuli in het receptieve veld (RF) van een neuron worden gepresenteerd, zou deze moeten verminderen op het moment dat stimuli verder vit elkaar worden geplaatst, zodat minder stimuli aanwezig zijn binnen een gegeven RF. Voorts neemt RF grootte toe naarmate men verder komt in de visuele hiërarchie, waardoor stimuli vaker binnen één RF zullen vallen in hogere visuele gebieden als V4 en TEO. In overeenstemming met deze hypotheses observeerden wij met functionele Magnetic Resonance Imaging ( $f M R I$ ) dat de grootte van de competitie tussen stimuli in de menselijke visuele cortex afneemt naarmate de stimuli verder vit elkaar staan. Verder werd meer competitie waargenomen naarmate men in hogere gebieden kijkt. Deze resultaten bevestigen dat "biased competition" als dominant selectiemechanisme optreedt in de menselijke visuele cortex.

Terwijl de fMRI-resultaten uit hoofdstuk 2 met hoge spatiële resolutie aantonen waar in de visuele cortex competitie plaatsvindt, geeft de hoge temporele resolutie van eventrelated potentials (ERP) die gebruikt werden in hoofdstuk 3 inzicht in het tijdsverloop van deze interacties. Door fluctuaties in elektrische spanning op de schedel te meten als reactie op gelijktijdig gepresenteerde stimuli, namen wij waar dat in een vroeg tijdsvenster

120 (tussen ongeveer 100 en 150 ms) na stimuluspresentatie, stimuli met elkaar strijden om neurale hulpbronnen, onafhankelijk van de locatie van aandacht. Slechts nadat hogere gebieden een eerste analyse van de stimuli voltooid hebben, komt aandacht in het spel om één van de stimuli te bevoordelen. Deze resultaten behoren tot de eersten die inzicht geven in de temporele aspecten van competitie in de menselijke visuele cortex.

Het feit dat stimuli met elkaar strijden om neurale representatie roept de vraag op wat er precies gebeurd met genegeerde stimuli. In hoofdstuk 4 wordt een studie beschreven waarin onderzocht werd of corticale gebieden die betrokken zijn bij phonologische verwerking, zoals het gebied van Wernicke, de inferieure frontale gyrus en de insula, 
representaties bevatten van de phonologische eigenschappen van genegeerde stimuli. Proefpersonen zagen twee plaatjes waarvan de namen phonologisch aan elkaar gerelateerd waren (bijv. 'vos' - 'vork'), danwel ongerelateerd (bijv. 'vos' - 'hark'). Deelnemers richtten hun aandacht of op één van deze plaatjes, of deden een controletaak waarin de plaatjes werden genegeerd. Het idee hierachter was dat als het brein de phonologische relatie tussen beide plaatjes detecteert, het de phonologische eigenschappen van beide plaatjes geactiveerd moet hebben. In overeenstemming met dit idee vonden wij dat het brein onder sommige taakomstandigheden anders reageert op gerelateerde dan op ongerelateerde plaatjes. Dit geeft aan dat de plaatjes geïdentificeerd worden en hun namen geactiveerd, zelfs als zii genegeerd worden.

De resultaten in hoofdstuk 4 gaven aan dat phonologische eigenschappen van genegeerde stimuli geïdentificeerd kunnen worden door het brein, maar het paradigma kan dat daarvoor gebruikt werd kan niet gebruikt worden om de sterkte en richting van competitie te meten in gebieden buiten de visuele cortex. Er was dus een paradigma nodig om selectieprocessen te meten in linguïstische gebieden zonder dat daarbij visuele competitie optreedt tussen stimuli. Een paradigma dat hieraan voldoet is beschreven in hoofdstuk 5, waar woorden letter voor letter op het scherm gespeld werden, en waar de hoeveelheid woorden die met deze letters gevormd konden worden gemanipuleerd werd. Het voordeel van dit design was dat het mogelijk was om de hoeveelheid woorden die geïnhibeerd dienen te worden te manipuleren wanneer een nieuwe letter gepresenteerd werd, zonder aandachtsvoordelen of visuele verschillen tussen stimuli te introduceren, zoals in hoofdstuk 4. Wii observeerden twee ERP componenten waarvan de grootte afhing van de hoeveelheid te inhiberen woorden. Dit geeft voor het eerst aan dat processen van lexicale selectie geobserveerd kunnen worden in het ERP, die afhankelijk zijn van het aantal te inhiberen lexicale kandidaten, in plaats van het aantal geactiveerde.

Samenvattend beschrijven de studies in dit proefschrift experimenten om de rol van selectiemechanismen in het menselijke brein te onderzoeken, zowel in gebieden voor visuele verwerking (hoofdstuk 2 en 3), als in linguïstische gebieden (hoofdstuk 4 en 5). Door methoden met een hoge spatiële resolutie (hoofdstuk 2 en 4) en die met een hoge temporele resolutie (hoofdstuk 3 en 5) te gebruiken, ontstaat een constructief beeld van het karakter van selectiemechanismen in het menselijke brein. Een discussie hiervan, alsmede praktische kanttekeningen, toekomstperspectieven en algemene opmerkingen worden besproken in hoofdstuk 6. 


\section{Acknowledgments}

A Ph.D. thesis does not write itself. Nor does one write it alone. Even though it were my fingers who typed the work that's lying in front of you, its creation depended on many people. Their (your) inputs ranged from scientific and existential contributions to creative, relaxing and personality-building ones. The funny thing is that most of these people are probably not even aware of their contributions. That's why l'd like to take a few words to thank them here. This list is by no means exhaustive, and is presented in no particular order besides making use of the psychological primacy and recency effect for the first and last entries.

Allereerst en allerbelangrijkst, gaat mijn dank vit naar mijn ouders. Pap en mam, zonder jullie was ik hier niet geweest. Dat geldt niet alleen voor het hier zijn in de fysieke zin van het woord. Jullie onvoorwaardelijke steun wat betreft zowel mijn studie als mijn persoonlijke leven betekent ontzettend veel voor me en ik ben jullie ontzettend dankbaar dat jullie mij de kans gegeven hebben om mij te ontwikkelen tot wie ik nu ben. Bedankt voor een ideale vitgangssituatie.

Hein en Gijs, big bro' en lil' bro', ook al hebben jullie wat minder bijgedragen aan mijn fysieke bestaan, ook jullie zijn in grote mate verantwoordelijk voor mijn ontwikkeling. Vroeger door het bouwen van boomhutten of het elkaar met broederlijke liefde in de haren vliegen, nu door het stellen van stimulerende en/of kritische vragen als 'wat is creativiteit nou eigenlijk?' (Hein), of 'wa kende daor nou mee?' (Gijs). Hein, ook super bedankt voor je hulp met het design van mijn proefschrift. Ook al is de inhoud het belangrijkste, het oog wil ook wat, en dat heeft het nu dankzii jou.

Mijn tijd in Maastricht is natuurlijk onlosmakelijk verbonden met de mensen die ik hier heb leren kennen. Jeroen en Lesya, Sietze en Natascha, Stefan J., Steffi, Frouke, Bastiaan E., Barbara, Arie en Jane (ik schaar jullie voor het gemak even in het Maastrichtse rijtie), Michael, Henk, Vincent, Sven, ik heb erg genoten van onze eetafspraken, wijnavonden, wandelingen, spelletjesavonden, battle nights, squash-dates en inspirerende gesprekken. Boukje, ook al hebben onze wegen zich nu gescheiden, een groot deel van mijn Aio-tijd heb ik met jou doorgebracht en ik heb veel aan je gehad op zowel werk- als persoonlijk gebied. Wout en Annedien, bedankt voor de goede zorgen tijdens de afronding van mijn proefschrift. Het was fijn om te weten dat ik altiid mijn hapje bij jullie kon scoren als ik weer te lang op de uni had gezeten (wat trouwens ook geldt voor Jochen en Alard, thanx guys!) en het was heerlijk om met jullie oude of nieuwe koeien uit de sloot te halen. Met Wout natuurlijk vrij oude koeien, met name waar ze betrekking hadden op de afwisselend intelligente en knotsgekke gesprekken van de aloude Eersel-gang. Dirk, Koen, Stefan, Sander (en Wout dus): bij deze ook voor jullie een eervolle vermelding. Margriet, of het nou een wijnrijk, vegetarisch dineetje is, een cultureel avondje, een ijsje in de stad, of een wandeling door de Limburgse heuvels, afspraken met jou vervelen nooit, en je 
enthousiasme en onbegrensde nieuwsgierigheid werken aanstekelijk. Dat laatste geldt zeker ook voor Joris. Een bergwandeling met jou is de ultieme manier om eerst je hoofd leeg te krijgen van alledaagse beslommeringen, en hem vervolgens weer vol te stoppen met de meest viteenlopende vragen. Ik zie ontzettend uit naar alle nog volgende vakanties, concerten, gesprekken, spelletjesavonden, discussies en afvraagsessies. Maastricht is niet meer hetzelfde sinds jii weg bent!

To my colleagues in Maastricht: I really enjoyed my time here, and most of the scientific values I hold today, were shaped by you. In addition, I really enjoyed the musical sessions, parties, soccer matches (played and viewed), barbecues, kolonisten-, poker- and starcraft-evenings, NC-productions, coffee breaks and other social gatherings. Due to the department's great success, there's too many of you now to credit everyone, but I'm sure you'll recognise yourselves in some of the 'enjoyments' described above.

And what is a scientist without the proper facilities? I'm very grateful for the research environment in Maastricht. Many thanks to Jacques' computer emergency squad (especially Boudewijn and Maurice), always ready to assist when computers do what they generally do: generate errors. The same holds for the guys at the technical service for keeping the labs running and to the administrative people who make sure that we can focus on scientific numbers rather than financial ones. In this respect I would specifically like to thank Annemie who always knows what's going on, what needs to be done and why, when and by whom. Without you, the department would be a mess (although you might say it already is). Although not sure whether to list them under 'facilities' or not, I'm very grateful to the Dandy Warhols for making such fantastic music, a necessary (but not sufficient) feature for creating 'flow'.

A special word of thanks is reserved for the people with whom I had the pleasure of working with more closely on the projects described in this thesis. Many thanks to my coauthors Arjen, Jens and especially Peter de W. It was a pleasure working with you guys. Steffi, Edwin and Ruben, thanks a lot for your help with data collection and analysis! Anke $\mathrm{H}$., thanks for your fruitful insights and brainstorms, and of course to you and Malte for the cosy beer nights and pointing out the differences between the Dutch and the Germans. Francesco G., your joining the project stimulated lots of discussions, but always combined with loads of smiles and laughter. I am very grateful to Rainer: promoter, fMRI guru, and host of many a party. The fact that a day (unfortunately) only has 24 hours is at least partly compensated by your quick way of thinking, and your creative views on science work inspiring. I've learned many things from you, and hope to be able to work with you in the future.

Special gratitude goes to Alard, faithful roomy and friend since a couple of years now, and sort of a personal help desk in my early ventures into the world of fMRI. I'll miss your humor, your know-how, the eternal struggle who could create the highest pile of junk on his desk, and the movie- and game nights. The same holds for Jochen. Although 
a later 'acquisition' here in Maastricht, we've grown close rapidly. Your 'no mountain too high'-philosophy of life is inspiring, and works great both literally and as a figure of speech. I really enjoyed those evenings with a perfect ratio of good food and wine, serious talks and laughter, and kicking each other's butt in some game or a match of floorball.

Which brings me, of course, to all the boys and girls at Hooked. There's no better way to clear your mind after a day of work than to run your ass off. And boy, that's what these guys do: make you run your ass off. Being totally unfamiliar with floorball when I came to Maastricht, it's now one of my favourite pastimes. Not in the least due to the atmosphere in the club. A special note here for Erkki. You basically transformed Hooked from a provincial little club to a thriving national champion and you were the driving force behind many social events. Even though you can't "be near" us at the moment, you're not forgotten! In short, floorball is just a great way to ease your mind, exercise, have fun, and get to meet lots of interesting people. And you never know, you might meet this one, very special person there. Someone lovely and caring. Someone who is there for you when you need her, always able to make you smile, and who never ceases to amaze you. Wietske, iij bent zo iemand. Bedankt voor de afgelopen tijd. Onze 'persoonlijke deadline' heeft de laatste loodjes een stuk makkelijker gemaakt en ik geniet ontzettend van het invullen en vitvoeren van ons 'things to do'-lijstje.

And then, as the proverb goes, last but definitely not least, my eternal gratitude goes to Bernie, the 'other woman' in my life. If I counted correctly, I've been working with you for about 7 years now, first as a student assistant, then for my masters thesis, and now the Ph.D. thesis. You fuelled my interest for science, gave me the opportunity to become a researcher, taught me how to be one, gave me the freedom to explore my own ways, braked me if I veered off too much and became a close friend in the process. I love your 'think out of the box'-attitude, your never-failing way of making people enthusiastic and I appreciate your unconditional faith in me. Although you've been trying to suppress all thoughts about it, it's now time for me to set out and explore what else is out there. But wherever I might end up, it all started here. Thank you for that. 


\section{Curric ulum vitae}

Mart Bles was born in Eersel, the Netherlands, on June 2nd 1980. He attended the Rythovius College, where he obtained his Atheneum diploma in 1998. He commenced to study psychology at Maastricht University in 1998, where he became a teaching assistant in 1999. From 2000 onwards, he worked as a research assistant on ERP studies on the time course of language production, together with his later supervisor, Bernadette Jansma and with Niels Schiller. He graduated in 2002, with a specialisation in developmental psychology (cum laude). He was employed as a Ph.D. student in 2003 at the department of Cognitive Neuroscience at Maastricht University. Since July 2007, he is a post doc research fellow at the Bernstein Centre for Computational Neuroscience in Berlin. 